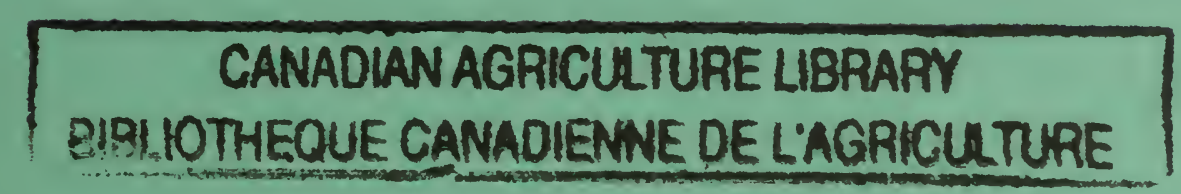

\title{
Water Relations and Forest Distribution in the Douglas-fir Region on Vancouver Island
}

\author{
R. G. McMinn
}

Forest Biology Division CANADA DEPARTMENT OF AGRICULTURE

Ottawa, Ontario 


\title{
Water Relations and Forest Distribution in the Douglas-fir Region on Vancouver Island
}

\author{
R. G. McMinn
}

\author{
Forest Biology Laboratory \\ Victoria B.C.
}


Copies of this publication may be obtained from Information Division

GANADA DEPARTMENT OF AGRICULTURE

Ottawa, Ontario

ROGER DUHAMEL, F.R.S.C.

QUEEN'S PRINTER AND CONTROLLER OF STATIONERY

OTTAWA, 1961

Cat. No. A43-1091

$2 \mathrm{M}-27087-4: 60$ 


\section{CONTENTS}

PAge

INTRODUCTION . . . . . . . . . . . . . . . . . . . . 5

Methods

Climate.............................. 10

Vegetation............................ 11

Microclimate................................ 12

Soils

Profile characteristics................... 12

Moisture regimes..................... 13

RESULTS

Climate............................... 15

Vegetation............................. 17

Microclimate........................... 31

Soils

Profile characteristics................... 36

Moisture regimes...................... 46

Discussion.................................... 60

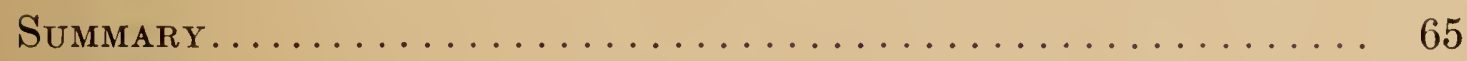

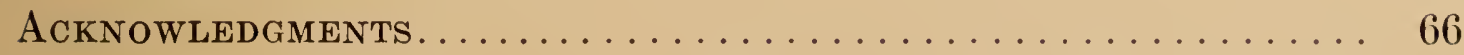

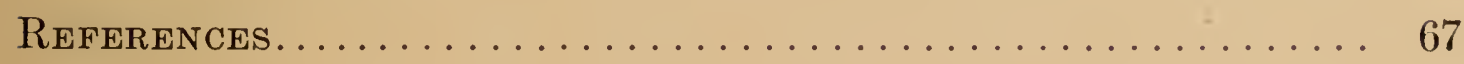

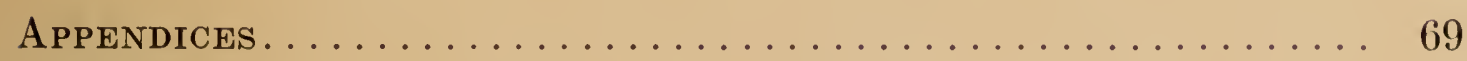





\section{INTRODUCTION}

Water relations play an important role in the distribution of vegetation in regions with low summer rainfall because the availability of water is commonly a limiting factor. Pearson (32), for example, concluded that the distribution of certain forest types in the south-western United States was largely determined by the presence or absence of soil drought and differences in its time of occurrence. McMinn (29) reached a similar conclusion for various plant associations in the northern Rocky Mountains. Even when available soil moisture has not been completely exhausted, the productivity of plants is influenced by deficits, that is by the reduction of soil moisture to amounts less than field capacity (35). The present study was undertaken to evaluate the significance of water relations in the differentiation and distribution of forest associations within the Douglas-fir (Pseudotsuga menziesii ${ }^{1}$ ) region on Vancouver Island, British Columbia ${ }^{2}$.

The Douglas-fir region of coastal British Columbia may be defined as the region in which Douglas-fir stands can predominate on most sites. This region is approximately coincident with the Southern Pacific Coast and Strait of Georgia Sections of the Coast Forest Region described by Rowe (39). Krajina $(20,21)$ recognizes within the Douglas-fir region a Coastal Douglas-fir Zone in which Douglas-fir is considered to be the climatic climax dominant. This Zone corresponds to the Strait of Georgia Section of the Coast Forest Region (39). Elsewhere in the Douglas-fir region, and even on some sites within the Coastal Douglas-fir Zone, the prevalence of Douglas-fir stands appears to be related to the periodic occurrence of forest fires, promoted by the hot, dry summers characteristic of the rainshadow climate of this region (40). Without such fires, Douglas-fir forests occurring outside the driest sections of the Douglas-fir region might eventually have been replaced by stands of more shade tolerant species, such as Thuja plicata (western red cedar) and Tsuga heterophylla (western hemlock). Various forest associations occurring within the Douglas-fir region of coastal British Columbia have been defined by Krajina (20).

The area selected for study was a portion of the Nanaimo River Valley, some eight miles southwest of Nanaimo, British Columbia (Fig. 1). The Nanaimo River is one of a series of easterly flowing streams arising in the central height of land which runs the length of Vancouver Island. The drainage basins of these rivers constitute much of the Douglas-fir region of coastal British Columbia. The study area (Fig. 2) consisted of a section of the valley extending seven miles downstream from Fourth Lake, which lies approximately twelve miles inland from the east coast of Vancouver Island. The Nanaimo River drops gradually from $1,000 \mathrm{ft}$. above sea level near Fourth Lake to $600 \mathrm{ft}$. above sea level east of First Lake. West of Second Lake, the valley floor is less than onehalf mile wide and its sides rise steeply for more than 2,000 ft. (Fig. 3). Many of the peaks are over 4,000 ft. high. The topography of this western portion of the study area is typical of the terrain of the central mountainous area of Vancouver Island. To the east of Second Lake, the valley widens and its sides rise less steeply. This section is characteristic of the less rugged topography of eastern Vancouver Island.

${ }^{1}$ Nomenelature is that of Little (25) for trees, Peek (33) for other vaseular plants, Conard (8) for mosses, and Howard (18) or Fink (11) for lichens, except where other authorities are given.

${ }^{2}$ This report is based on a thesis submitted in partial fulfilment of the requirements for the degree of Doctor of Philosophy in the Department of Biology and Botany, University of British Columbia. 


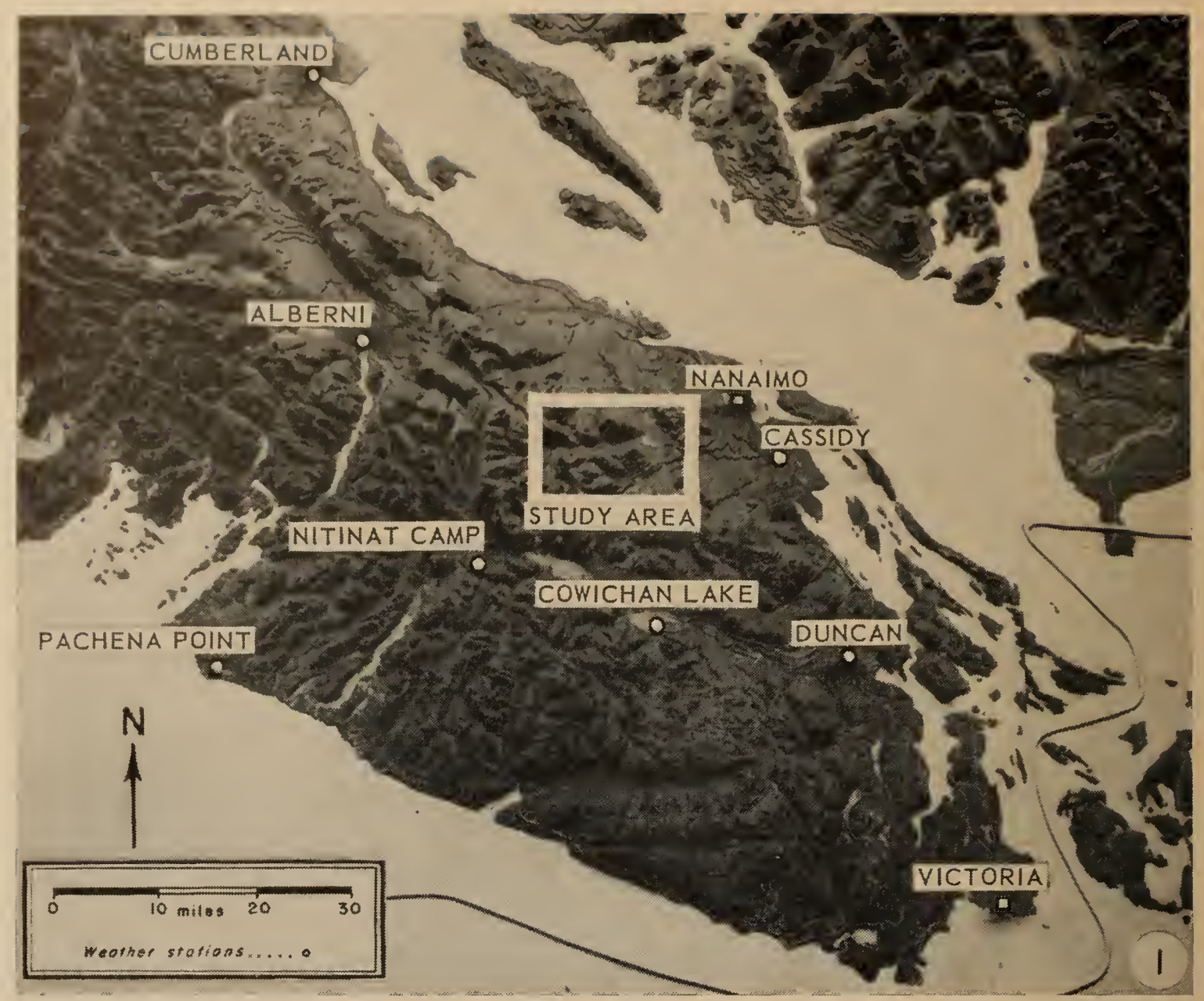

FIGURE 1-Map of southern Vancouver Island, showing location of study area and various weather stations. The mountainous topography of the west coast and central regions of the Island contrasts with the less rugged terrain of the eastern side. Vertical scale is increased five times.

The dominant tree canopy of most stands in the Nanaimo River Valley originated as a more or less even-aged stand following a forest fire. Within the study area, the age of the oldest trees of the dominant canopy varied from 220 to 320 years (Table I), suggesting their establishment following several different fires. Some stands were evidently also affected by subsequent fires, the most recent of which occurred about 65 years prior to the present study (41). These recent fires had little effect on Pseudotsuga menziesii, a relatively fireresistant tree with a deep root system and a thick bark at maturity. On the other hand, even ground fires may kill Tsuga heterophylla and Thuja plicata, species which have thin bark and shallow root systems. The Tsuga heterophylla and Thuja plicata understoreys of many stands in the Nanaimo River Valley were of a younger age class than their Pseudotsuga menziesii overstoreys. Such understoreys may have originated following fires which occurred after the establishment of the overstorey. These fires presumably also influenced the composition of subordinate plant layers and the depth of litter layers.

The following associations were studied:

Pseudotsuga menziesii-Pinus contorta-Gaultheria shallon-Peltigera membranaceaPeltigera aphthosa association (Pseudotsuga-Gaultheria-Peltigera association $\left.^{3}\right)$;

${ }^{3}$ The designation of associations is according to the classification proposed by Krajina (20). The abbreviated names shown in parentheses are used in all subsequent reference to the various associations. 


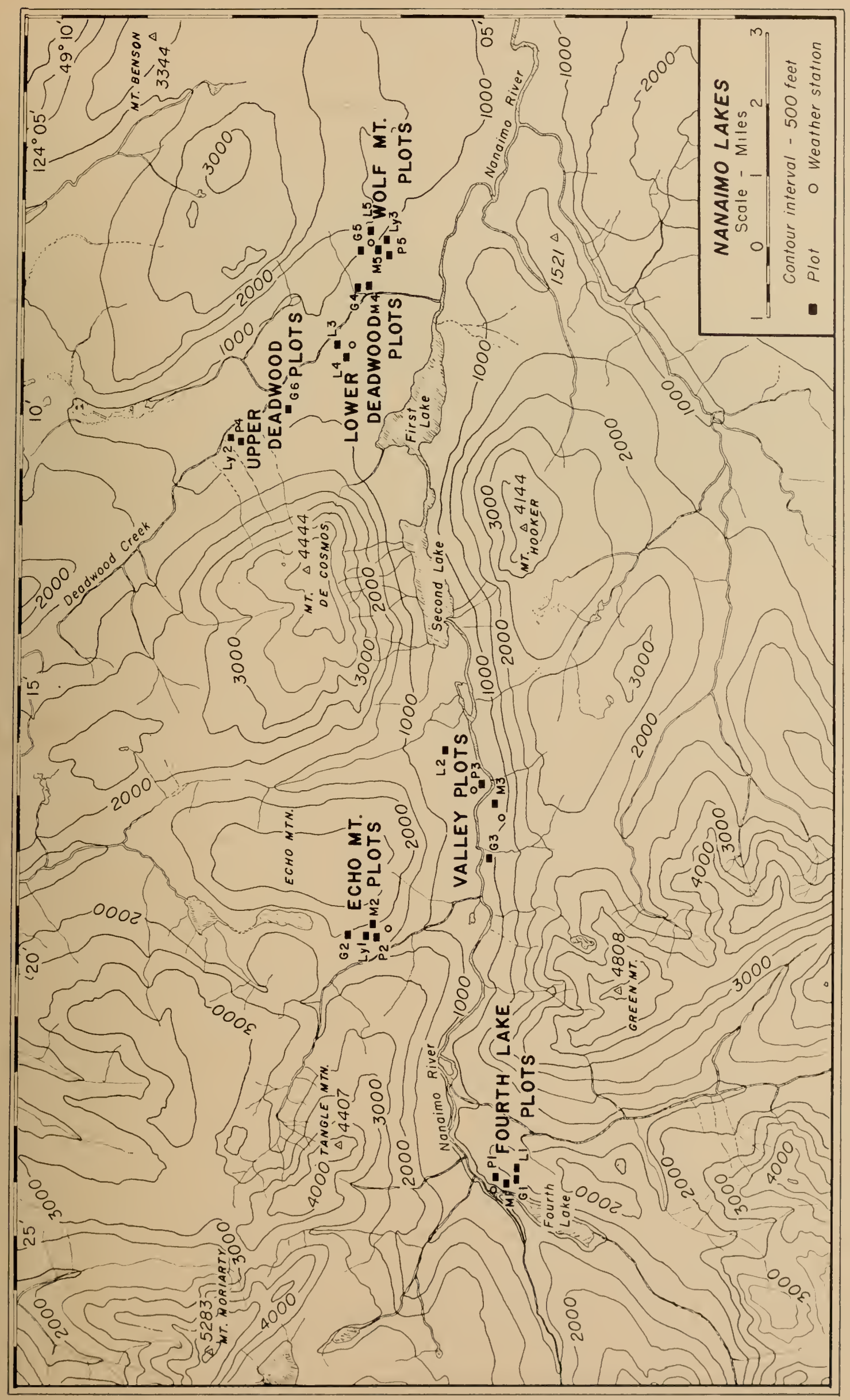

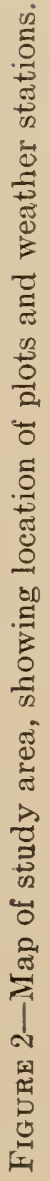




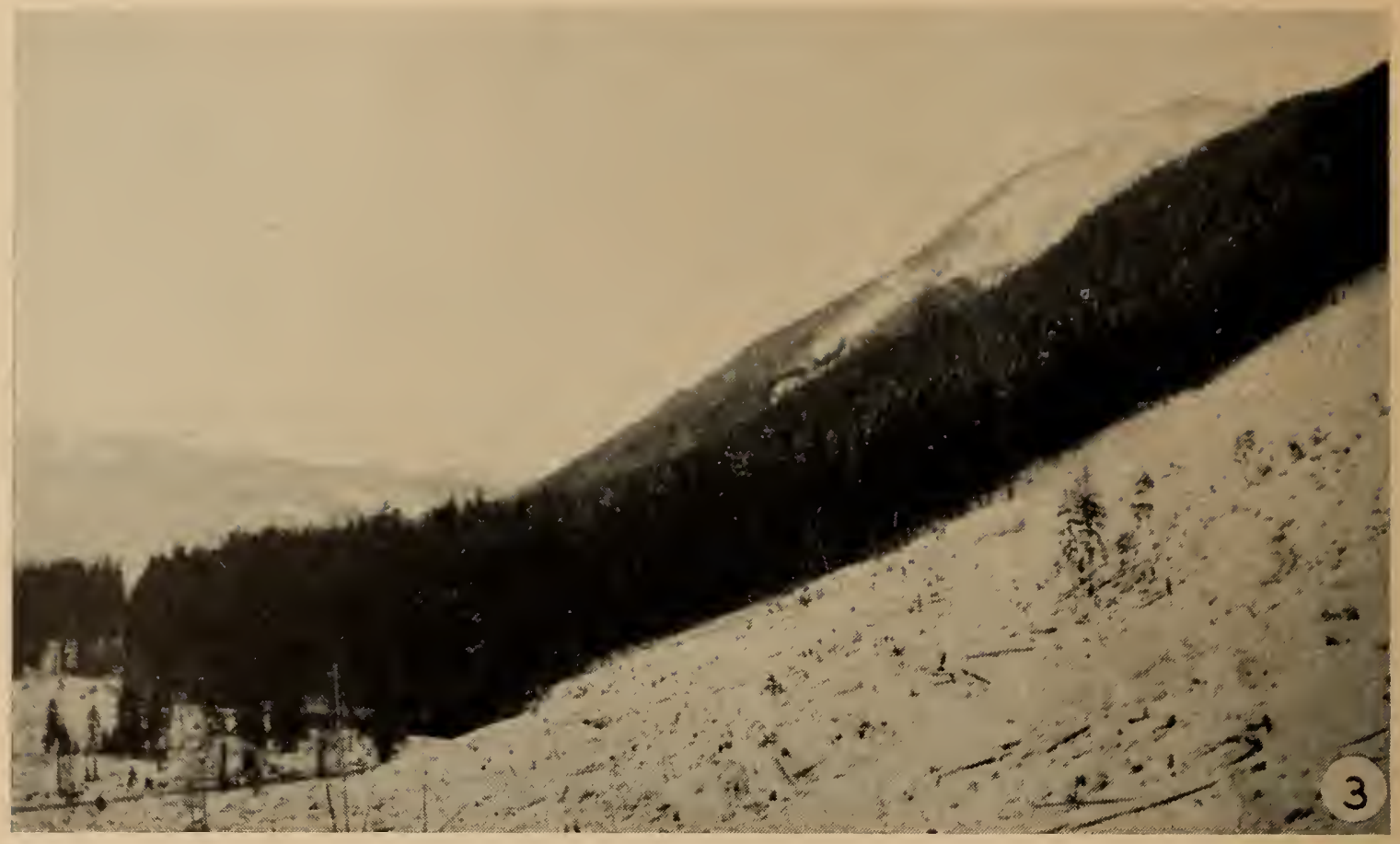

FIgURE 3-A typical hillside in the Nanaimo River Valley, showing the variation in tree heights from upper slopes to valley floor. This change reflected the sequence of forest associations.

Pseudotsuga menziesii-Gaultheria shallon association (Pseudotsuga-Gaultheria association);

Pseudotsuga menziesii-Tsuga heterophylla-Gaultheria shallon association (Pseudotsuga-Gaultheria association);

Pseudotsuga menziesii-Tsuga heterophylla-Hylocomium splendens-Eurhynchium oreganum association (Pseudotsuga-Tsuga-Hylocomium association), which occurs as a subassociation typicum and a subassociation nudum:

Pseudotsuga menziesii-Thuja plicata-Polystichum munitum association (Pseudotsuga-Polystichum association);

Thuja plicata-Lysichitum americanum association (Thuja-Lysichitum association).

In the easterly portion of the study area, ridges at the top of hillsides characteristically supported stands of the Pseudotsuga-Gaultheria-Peltigera association, with Pseudotsuga-Gaultheria stands occuring on upper slopes, Pseudotsuga-Tsuga-Hylocomium stands at mid-slope, Pseudotsuga-Polystichum stands on lower slopes and the valley floor, and Thuja-Lysichitum stands in swampy bottomlands (Fig. 4). Local variation from this catenary distribution of stands was, nevertherless, frequent. In the more mountainous western portions of the valley, Pseudotsuga-Tsuga-Gaultheria stands replaced the PseudotsugaGaultheria association and still other associations occurred at high elevations. Even at low elevations, associations other than those studied were present. A Thuja plicata-Abies grandis-Adiantum pedatum association, for example, was found on occasionally-flooded alluvial benches, an Arctostaphylos uva-ursi association occurred on treeless rock outcrops, and Sphagnum spp. characterized stagnant swamps (20). Other Douglas-fir types have also been described from Washington and Oregon (2). The associations covered in this study, nevertheless, represent some of the most prevalent site classes and commercial timber types of the Douglas-fir region on Vancouver Island. 


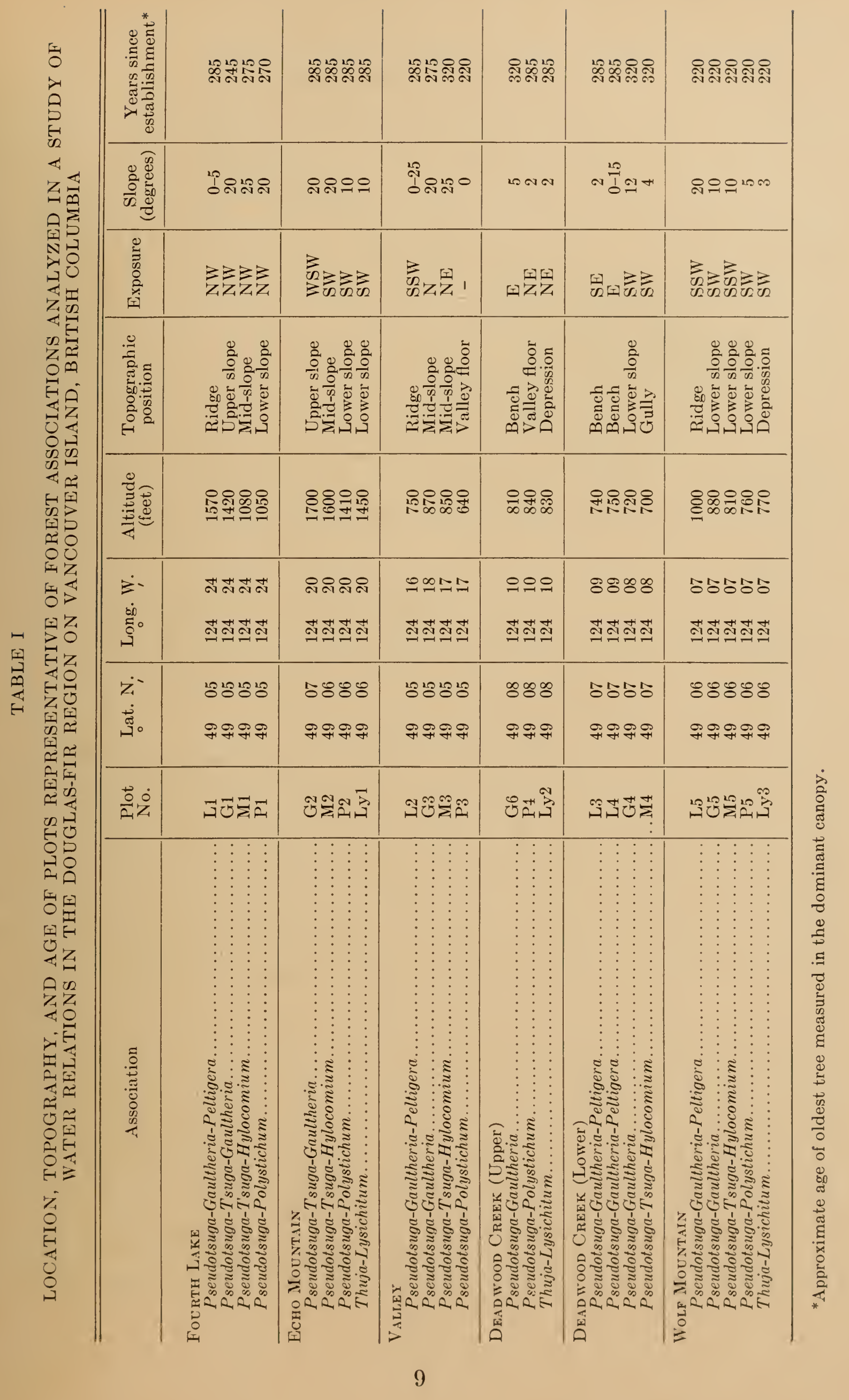




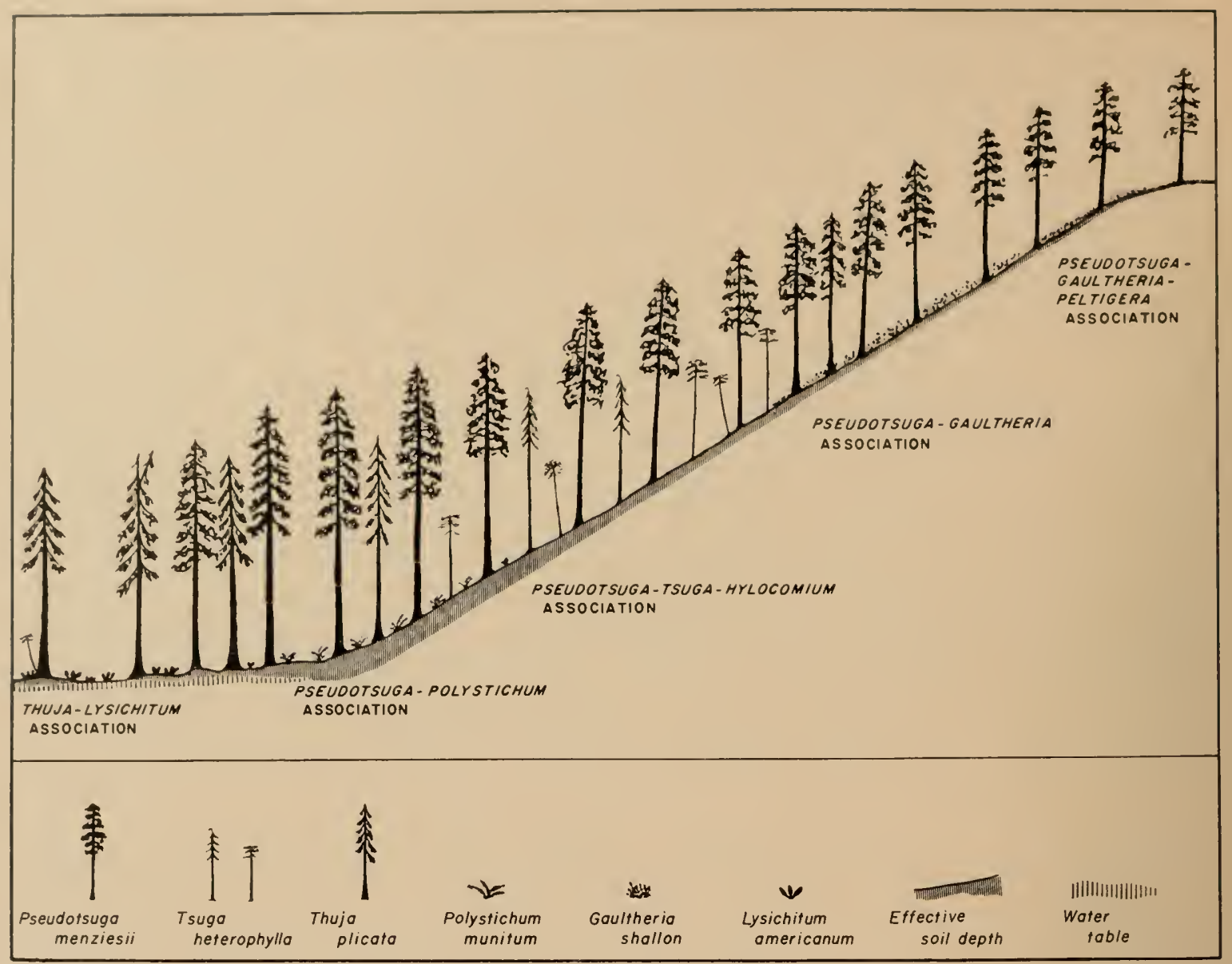

Figure 4-Sequence of forest associations in the Douglas-fir region on Vancouver Island.

\section{METHODS}

Groups of plots (Fig. 2, Table I) were selected in mature stands at various locations along the valley so that comparisons could be made between both plots representing different associations under similar climatic conditions and plots representing the same association under different climates. Since plots were to be visited at frequent intervals, accessibility was important and restricted the study to areas served by logging roads. This limitation meant that some of the plots chosen were situated in atypical positions in the catenary sequence. All plots, however, were considered to be representative of their association. Twenty-four plots, each of one-quarter acre, were analyzed.

\section{Climate}

Precipitation, and maximum and minimum temperatures were recorded at monthly intervals to indicate the climate of the six localities in which plots were situated. Recording stations were located in clear-cut areas at least $50 \mathrm{~m}$. from the standing timber. Measurements were made from June, 1951, through December, 1956. Data for other locations on Vancouver Island were obtained from the published records of the Meteorological Division of the Canada Department of 'Transport and records taken at the Nitinat Camp of the British Columbia Forest Products Company Limited.

Rain gauges with an orifice of $185 \mathrm{sq} . \mathrm{cm}$. ( 6 in. in diameter) were used for precipitation measurements, since a large orifice size has been shown to minimize inaccuracies due to wall effect (30). Evaporation of the water collected between 
readings was restricted by the addition of paraffin oil to the reservoirs. Inaccuracies resulting from turbulence about the orifice were reduced by placing the rain gauges in small pits with their orifices almost flush with ground line (38). In winter, extra sleeves were added to bring the orifice of the gauges above the anticipated depth of snow. These sleeves prevented snow from drifting into the gauges, although they had the disadvantage of increasing turbulence about the orifice. Any ice or snow accumulated during winter months was melted with a portable gasoline stove before measurements were made.

Initially the Six's type maximum and minimum thermometers used for recording temperatures $1.5 \mathrm{~m}$. above ground level were housed in improvised shelters. During 1955 and 1956, temperatures were also recorded in standard Stevenson screens. Comparison of records for these years showed that maximum values from the improvised shelters were higher than those from the Stevenson screens during warm summer months. Summer values consequently are based only on records from the Stevenson screens. Winter values, on the other hand, are based on the entire 1952-56 record because maximum and minimum values from both types of shelter corresponded closely during this season.

\section{Vegetation}

The trees and subordinate plants (shrubs, herbs, mosses, and lichens) in each plot were analyzed by various techniques appropriate to the different layers. Subordinate plant layers were analyzed during July and early August when all plants were fully developed ${ }^{4}$.

The diameter at breast height ( $4.5 \mathrm{ft}$.) of all trees over $4 \mathrm{in}$. in diameter outside the bark was measured, together with the height and age of trees of each species representative of the diameter classes present. Other tree heights were then obtained from the height/diameter curves constructed for each species in each plot. The average height of the dominants and codominants of each species were determined by averaging the height of the tallest 55 per cent of the trees of each species. Site index for Douglas-fir was determined from the curves developed by McArdle, Meyer, and Bruce (28). Volumes per acre were derived from volume tables prepared by the Forest Surveys Division of the British Columbia Forest Service (13).

The density of plants in the shrub layer was determined by line interception (5). Ten lines, each $50 \mathrm{ft}$. long, were run across the plots at approximately 6 - $\mathrm{ft}$. intervals. The length of line covered by a vertical projection of leaf surfaces was recorded and expressed as a percentage of interception. The line interception method as employed in this study involved some inaccuracy, since tall shrubs could not be viewed from directly above. However, any error seemed sufficiently small that the use of a plumb line did not appear warranted.

Plants of the herb layer were evaluated by the frequency method (4). The size of frequency frame used, 2 by $5 \mathrm{dm}$., was gauged to allow an appropriate number of species to fall within each frame. Since this size was too small to describe accurately the frequency of such large herbs as Polystichum munitum and Lysichitum americanum, these plants were also evaluated by the line interception method. In the case of ferns, the length of line covered by a circle described around the clump was recorded as the amount intercepted. Frequency frames were set at 5 -ft. intervals along the lines used for line interception. Eighty to one hundred frames were tallied on each plot and results were expressed as the percentage of frames in which a species occurred.

\footnotetext{
${ }^{4}$ The original specimens determined during vegetation analyses were destroyed by fire.
} 
Mosses and lichens growing on the ground were evaluated by the point frequency method (24). A 10-point frame was set at 5-ft. intervals along the line interecption tape. Eight hundred to one thousand points were examined on each plot and the frequency with which species were contacted was expressed as a percentage.

The abundance and coverage of mosses and lichens growing on decaying logs and the boles of trees up to a height of $2 \mathrm{~m}$. above the ground were estimated according to the 11-point total estimate scale of Krajina and Domin (19).

\section{Microclimate}

Precipitation that penetrated the tree canopy was measured by averaging collections from four rain gauges placed on each plot. Percentages of interception were calculated by comparing the amounts collected within plots with those collected in adjacent open areas. Records were maintained from June, 1951, until October, 1953. Since little or no water ran down tree boles during summer months, the only season when such water might be expected to influence soil moisture content, this component of precipitation was not measured.

During part of the study, nine additional rain gauges were placed on four plots of different associations to test the adequacy of the four-gauge sample. It was concluded that the four-gauge average was a satisfactory measure of the precipitation reaching subordinate plant layers because the smaller sample rarely varied from the larger by more than 1 or $2 \mathrm{~mm}$, a difference which could have no appreciable influence on soil-moisture regimes.

Porous porcelain atmometers (26) were used to compare evaporation rates in plots and at three of the adjacent open stations. Bulbs were placed at $5 \mathrm{~cm}$. and $100 \mathrm{~cm}$. above ground line. Entry of rain water into the reservoirs was restricted by the use of mercury valves (9). Each atmometer was standardized on a rotating table in the customary manner. Readings were taken twice monthly from June through September, 1951 and 1952. For simplicity, results were expressed as relative evaporation by converting each value to a percentage of the highest value recorded.

Maximum and minimum air temperature at $1.5 \mathrm{~m}$. above the ground were recorded on the Wolf Mountain and Fourth Lake plots at monthly intervals June to September, 1953.

Soil-surface temperatures were measured with Six's type maximum and minimum thermometers buried with their bulbs approximately $5 \mathrm{~cm}$. below the soil surface. In most cases this depth placed the bulbs just below the litter layer. Readings were made in all plots, twice monthly during the summer and at oneor two-month intervals during other seasons. Records were maintained from June, 1951, until November, 1953. The temperature of deeper soil horizons was measured whenever soil moisture was sampled. During direct sampling, measurements were made by inserting a thermometer into the side of the sampling pit at various depths. The thermisters incorporated in the fiberglas soil moisture units allowed simultaneous temperature measurements during indirect moisture sampling (7).

\section{Profile Characteristics}

\section{Soils}

Five or more soil profiles were examined in each plot. Descriptions were made in accordance with the terminology of the United States Department of Agriculture Soil Survey Manual (44), although some additional terms were used 
for humus layers and organic soils $(17,45)$. In addition to field descriptions, ten profiles were treated with vinylite resin to preserve them for laboratory examination (42).

Particle size distribution within the $2-\mathrm{mm}$. soil fraction was determined by the hydrometer method, according to the procedure prescribed by the American Society for Testing Materials (1). Textural classes were interpreted from the chart included in the Soil Survey Manual (44).

Sieved, air-dried soils were re-wet to a saturated paste for $\mathrm{pH}$ determinations (34). Measurements were made with a Beckman N-2 glass electrode meter.

\section{Moisture Regimes}

Periodic measurements of soil-moisture contents were made using both direct and indirect sampling methods. Samples for direct determinations were obtained by digging pits because the soils of most plots were too stony for the use of samplers. Samples were taken at 1-dm. intervals to a depth of $1 \mathrm{~m}$., or to the hardpan in shallow soils. Sample pits were refilled after use and new sample pits were dug at least $3 \mathrm{~m}$. distant from earlier pits. The moisture content of samples was determined by oven drying the $5-\mathrm{mm}$. soil fraction at $105^{\circ} \mathrm{C}$. and results were expressed as a percentage of dry weight. Direct sampling was used from July, 1951, until September, 1952.

Indirect measurements of soil moisture were continued from October, 1952, until November, 1953. Fiberglas units (7) were inserted into the side of pits at depths of approximately $5,15,30,50,80$, and $120 \mathrm{~cm}$. in deep profiles or down to the hardpan in shallower soils. This method of placement may have resulted in some soil-moisture values being too high because the number of living roots in the soil adjacent to units was doubtless reduced by the severance of some roots when pits were dug. Nevertheless, seasonal changes in moisture values appeared sufficiently marked to suggest that enough roots were left to bring about soil-moisture depletions to characteristic levels. One bank of units was placed in each plot. The rapid measurement of units was facilitated by connecting their leads to a rotary selector switch.

Interpretation of field records was made from calibration curves derived from substitute units because the original soil-moisture units were accidentally destroyed before their calibration had been undertaken. However, tests conducted on ten of the new units in a single test soil showed that variability among such fiberglas units was less than 1 per cent at low soil-moisture contents.

Each soil-moisture unit was calibrated against the $5-\mathrm{mm}$. fraction of the soil which surrounded the original soil-moisture unit in the field. The 5-mm. fraction was used for calibration because collection of the undisturbed cores recommended by Hendrix and Colman (16) was impractical in the coarse, gravelly soils of the study area. Moreover, sieved samples were thought to be satisfactory because wilting percentage is more dependent on soil texture and organic content than on structure, and the major emphasis in this study is on available soil-moisture content. The moisture contents of sieved samples are also considered to be more truly comparable with one another than contents derived from unsieved samples because in the latter case considerable variability may be introduced through differences in coarse gravel contents.

The cans used for the calibration of soil-moisture units contained approximately $150 \mathrm{gms}$. of soil. Uniformity of soil-moisture content in these cans was promoted by covering them with a loosely fitting lid during drying cycles. A drying cycle consisted of a series of warm periods at $35^{\circ} \mathrm{C}$. to expel moisture, each followed by an equilibration period of one or more days at $15^{\circ} \mathrm{C}$. Tests showed that following this procedure moisture contents were essentially uniform 
throughout the can. Can weights and the resistance reading of each unit were determined after each equilibration period. Following determination of the oven-dry weight of soil in the can, the moisture percentage corresponding to each resistance was calculated. The calibration curve plotted for each soil was based on at least four drying cycles, each consisting of six or more readings between saturation and wilting range.

Since the results of moisture samplings were to be expressed as percentages of available moisture, a wilting percentage was established for each of the nearly 2,200 simples collected. The 15-atmosphere percentage, as determined by a pressure membrane apparatus (36), appeared to be the most satisfactory value that could be obtained for such a large number of samples, even though some deviation between the two values has been reported (27). The procedure followed was similar to that outlined by Richards (34), except that the 5-mm. soil fraction was used. A standard sample of known moisture constant was included with each sample lot. When the value obtained for this standard differed from the true value by less than 2 per cent a correction factor was applied to the values obtained for the unknown samples in the lot. A new extraction was made when the difference was greater than 2 per cent. A 15-atmosphere percentage was determined on a subsample of the soil surrounding each fiberglas unit and each of the samples dug for direct measurement. Available-moisture percentages were found by subtracting the appropriate 15-atmosphere percentage from the field-moisture percentage.

While no laboratory procedure is entirely satisfactory for the measurement of field capacities, the $1 / 3$-atmosphere percentage appears to be a convenient value to indicate when soils are nearing saturation (37). The procedure followed was similar to that of Richards (34), except that extractions were made on the $5-\mathrm{mm}$. soil fraction and a Visking membrane was used. It was found that values obtained using this method corresponded closely to field-moisture contents determined after soils had drained to field capacity following heavy rainfall. The 1/3-atmosphere percentage of all very wet samples was determined to confirm whether gravitational water was in fact present. Observation pits on each plot were also inspected at intervals to determine the depth of any water table present.

The volume weight and depth of fine soil present in representative profiles were determined so that soil-moisture contents could be expressed as depths of available water. Sample volume for volume-weight determinations was found by measuring the volume of sand required to fill a plastic bag lining the cavity which remained following the removal of each sample. Volume weights were then calculated by dividing the oven-dry weight of each sample by its volume. Values from the five pits sampled at decimeter intervals on each plot were averaged. The amount of fine soil in each $2 \mathrm{dm}$. of a representative profile was found by sifting the $5-\mathrm{mm}$. fraction from samples of known cross-sectional area ( 2 by 5 $\mathrm{dm}$.) and measuring the volume of water it displaced. The depth of fine soil in each $2 \mathrm{dm}$. was calculated by dividing the volume displaced by the cross-sectional area of the sample.

The depth of available water present each time soil-moisture contents were sampled was calculated by multiplying the appropriate depth of fine soil in each $2 \mathrm{dm}$. of the profile by the percentage by volume of available soil moisture present (volume weight $X$ percentage by weight of available water $/ 100$ ). Assuming that the amount of water held by coarse soil fractions is negligible, the total depth of available water was found by summing the depths present in each $2 \mathrm{dm}$. of the profile. Values for the 30-month period during which soil-moisture contents were sampled were averaged to show the seasonal variation in depth of available water present in each plot. 


\section{RESULTS}

\section{Climate}

Summer months in the Nanaimo River Valley and other locations in the Douglas-fir region, for which long-term records are available, were characterized by potential water deficiences during the study period because summer rainfall was light and temperatures were relatively warm (Table II). Such weather seems quite typical, since the 1951-56 averages differed little from the long-term averages (Appendix I).

The low summer rainfall and relatively high summer temperatures characteristic of stations within the Douglas-fir region contrast with the higher rainfall and lower temperatures recorded at stations in the hemlock zone (21). The climate of Pachena Point, for example, on the west coast of Vancouver Island is typically maritime, while that of central mountain and east coast stations in the Douglas-fir region has a continental "tone". Such differences in precipitation occur because most rain-bearing winds come from the Pacific Ocean, placing areas to the lee of the mountainous west coast of Vancouver Island under the influence of a rainshadow effect (6). The width of this zone and the intensity of the rainshadow effect is, nevertheless, quite variable. During periods of high rainfall, such as in autumn and winter months, stations in the central mountain portion of the Douglas-fir region (for instance Nitinat Camp or Fourth Lake) seemed to be outside the rainshadow zone, for they received almost as much precipitation as Pachena Point in the hemlock zone (Table II). In summer months, on the other hand, rainfall at such stations amounted to little more than half that at Pachena Point. Presumably this westward displacement of the rainshadow climate in summer occurs because the relatively small moisture content of clouds passing the coastal mountains is soon depleted, leaving little to fall farther east. During the growing season, therefore, even the westerly plots in the study area were inside the rainshadow zone. That the intensity of the rainshadow effect increases from west to east is exemplified by records from Nitinat Camp, Cowichan Lake, and Duncan. Average rainfall, both in summer and the year round, at the east coast station (Duncan) was only half that measured at the westerly station (Nitinat Camp). A similar trend was demonstrable in the study area. Potential moisture deficiencies are, therefore, likely to be more extreme in the easterly portion of the study area than farther west. Cloudy weather also occurred less frequently in the easterly section of the valley than it did farther west.

Winter temperatures in the study area were usually sufficiently low for much of the December-through-March precipitation to fall as snow. However, the depth and duration of the snow pack in different locations varied considerably. During the period 1952 to 1956, the average maximum depth of snow at the Fourth Lake Station was 38 in., at the Valley Station it was 19 in., and at the Deadwood Creek Station it was only $8 \mathrm{in}$. Such variation reflected temperature as well as precipitation differences. On several occasions during visits to the study area to collect weather data, snow was falling at Fourth Lake, while only rain was encountered at Deadwood Creek. In the springs of both 1952 and 1954 , snow remained at the Fourth Lake and Echo Mountain Stations into April, by which time the ground was only partly covered in the Valley area and the Deadwood Creek area was already clear. Similarly, at the end of March, 1956, the snow pack was still near its maximum depth of 5 to $6 \mathrm{ft}$. at Fourth Lake and 3 to $4 \mathrm{ft}$. on Echo Mountain. The Valley Station, on the other hand, had little more than 6 in. and the Deadwood Creek area was largely free from snow. 


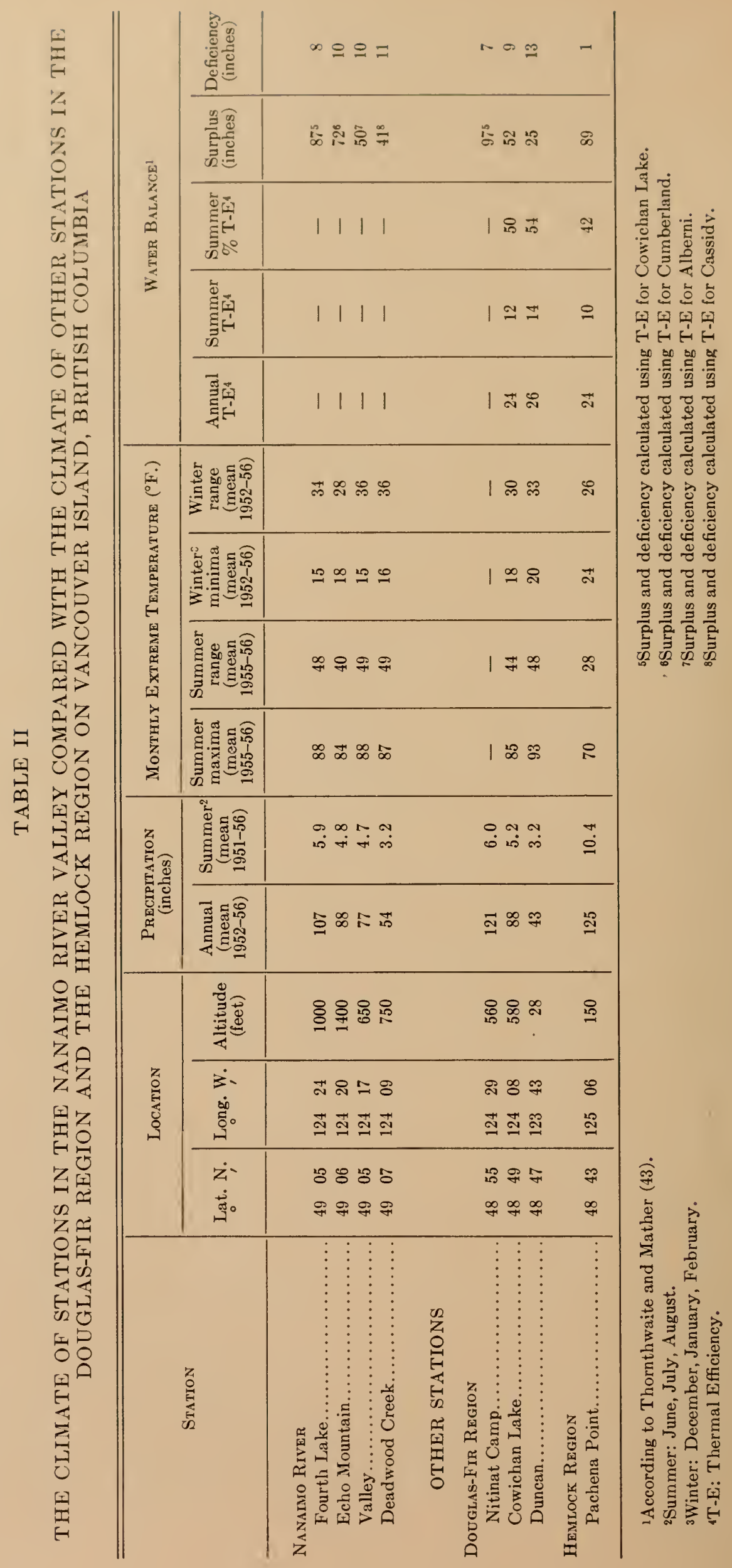


The growing season in the study area extended from early May until midOctober. At Fourth Lake and on Echo Mountain, however, the growing season appeared to be somewhat shorter than it was farther east, since phenological events in spring, such as the appearance and increase in size of new Polystichum munitum fronds, were generally one or two weeks later in occurrence.

\section{Vegetation}

Vegetation analyses confirmed that the composition and productivity of tree and subordinate plant layers in the plots studied were typical of their respective associations. Differences evident among plots of the same association seemed attributable to the influence of geographic location, topography, and the soil characteristics of the individual plot.

\section{Pseudotsuga-Gaultheria-Peltigera association}

Despite the relatively large number of Pseudotsuga menziesii per acre, their small height and diameter in the Pseudotsuga-Gaultheria-Peltigera plots resulted in stands with rather open canopies and low volumes per acre (Table III, Fig. 5). At Fourth Lake (Plot L1), volume per acre was further decreased by stand openings occupied by rock outcrops. Tree heights in this plot were also lower than those in the other Pseudotsuga-Gaultheria-Peltigera plots. No secondary canopy of intermediate trees occurred in any plot, although occasional suppressed Pseudotsuga menziesii, and, in some plots, Tsuga heterophylla, Pinus contorta, and $P$. monticola were present. The absence of a secondary canopy may have been at least partially attributable to ground fires subsequent to stand establishment. The presence of charred bark on the lower boles of several trees in each plot attested to the occurrence of such fires. Occasional clumps of Tsuga heterophylla up to $40 \mathrm{ft}$. high were present on most plots and individual Pinus contorta reached $60 \mathrm{ft}$. in Plot L4. All understorey trees, however, were less than 100 years old, some 100 to 200 years younger than most trees of the dominant canopy.

Gaultheria shallon, less than $6 \mathrm{dm}$. high, constituted the principal cover of shrub layers. Other species occurred only as scattered individuals. Clumps of Tsuga heterophylla, 4 to $5 \mathrm{ft}$. tall, were present in some plots, particularly those toward the western end of the study area. A few Pseudotsuga menziesii were also included within the shrub layer in the Wolf Mountain Plot (L5). Although a fairly large number of species was represented in the herb layer of most plots, only Linnaea borealis, Chimaphila umbellata, Mahonia nervosa, and Goodyera oblongifolia occurred with any considerable frequency. Camptothecium megaptilum, Calliergonella schreberi, Polytrichum spp., and Rhacomitrium spp., mosses characteristic of the association, formed extensive patches on some plots. Hylocomium splendens and Eurhynchium oreganum were common throughout. Various species of Cladonia, Peltigera, and Stereocaulon were also present amongst the ground cover plants, especially on the Fourth Lake Plot. Other lichens, particularly Alectoria sarmentosa, Sphaerophorus globosus, Parmelia physodes, and Cetraria spp. were a conspicuous feature on the bark of trees in all plots.

\section{Pseudotsuga-Gaultheria association}

The greater height and diameter of Pseudotsuga menziesii in the PseudotsugaGaultheria plots resulted in larger basal areas and volumes per acre than those of the Pseudotsuga-Gaultheria-Peltigera plots (Table III). A secondary canopy of suppressed Tsuga heterophylla and Thuja plicata was present in Plot G6, but these species occurred only as scattered clumps or individuals in other plots and they contributed little to stand volume. All trees of these species, however, were of a younger age class than the Pseudotsuga menziesii overstoreys in the plots analy zed. 


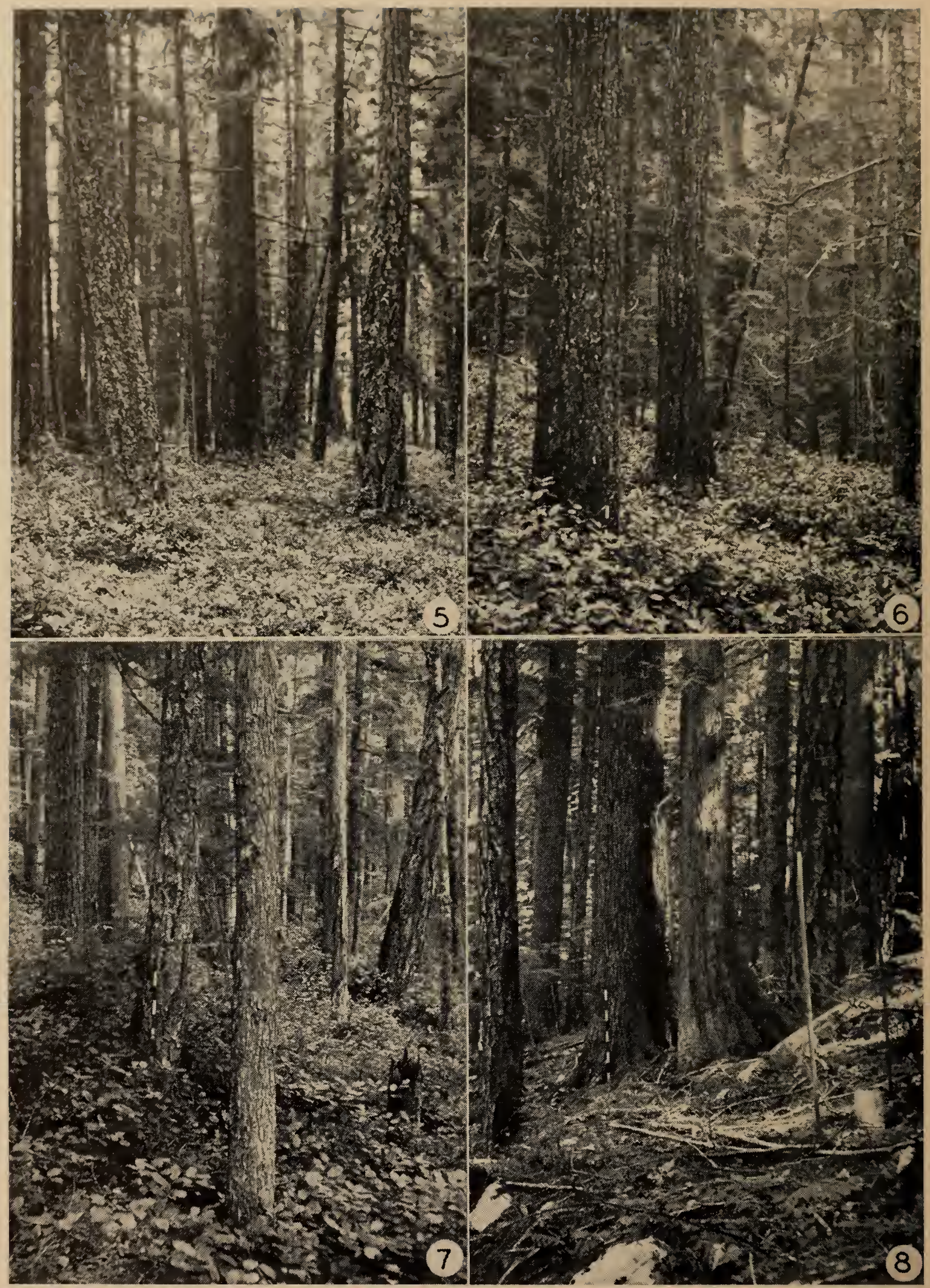

Figure 5-A stand of the Pseudotsuga-Gaultheria-Peltigera association, showing the sinall diameters of

Pseudotsuga menziesii and sparseness of Gaultheria shallon. The meter stick is marked in decimeters.

Figure 6-A stand of the Pscudotsuga-Gaultheria association. The increased diameters of Pseudotsuga menziesii and height of the Gaultheria shallon layer may be judged from the meter stick.

Figure 7-A stand of the Pseudotsuga-Tsuga-Gaultheria association, showing the small diameters of both Pscudotsuga menziesii and Tsuga heterophylla.

Figure 8-The Fourth Lake Plot of the Pseudotsuga-Tsuga Hylocomıum association, showing the comparatively large size of Tsuga heterophylla (beside meter stick). One of the rain gauges is evident in the right foreground. 
The Gaultheria shallon of the shrub layer was commonly over $1 \mathrm{~m}$. in height and considerably denser than in the Pseudotsuga-Gaultheria-Peltigera plots (Fig. 6). Other shrubs still had little cover value and herb layers were characterized by a poor species representation. The most common plants of herb layers in the Pseudotsuga-Gaultheria-Peltigera plots were also common in the Pseudotsuga-Gaultheria plots, but their frequencies were lower. Hylocomium splendens and Eurhynchium oreganum were the prominent mosses of mosslichen layers, and they covered the ground to the virtual exclusion of other species. Lichens were absent from the ground layer.

TABLE III

VEGETATION ANALYSES OF PLOTS REPRESENTATIVE OF FOREST ASSOCIATIONS IN THE NANAIMO RIVER VALLEY, BRITISH COLUMBIA

PSEUDOTSUGA-GAULTHERIA-PELTIGERA ASSOCIATION

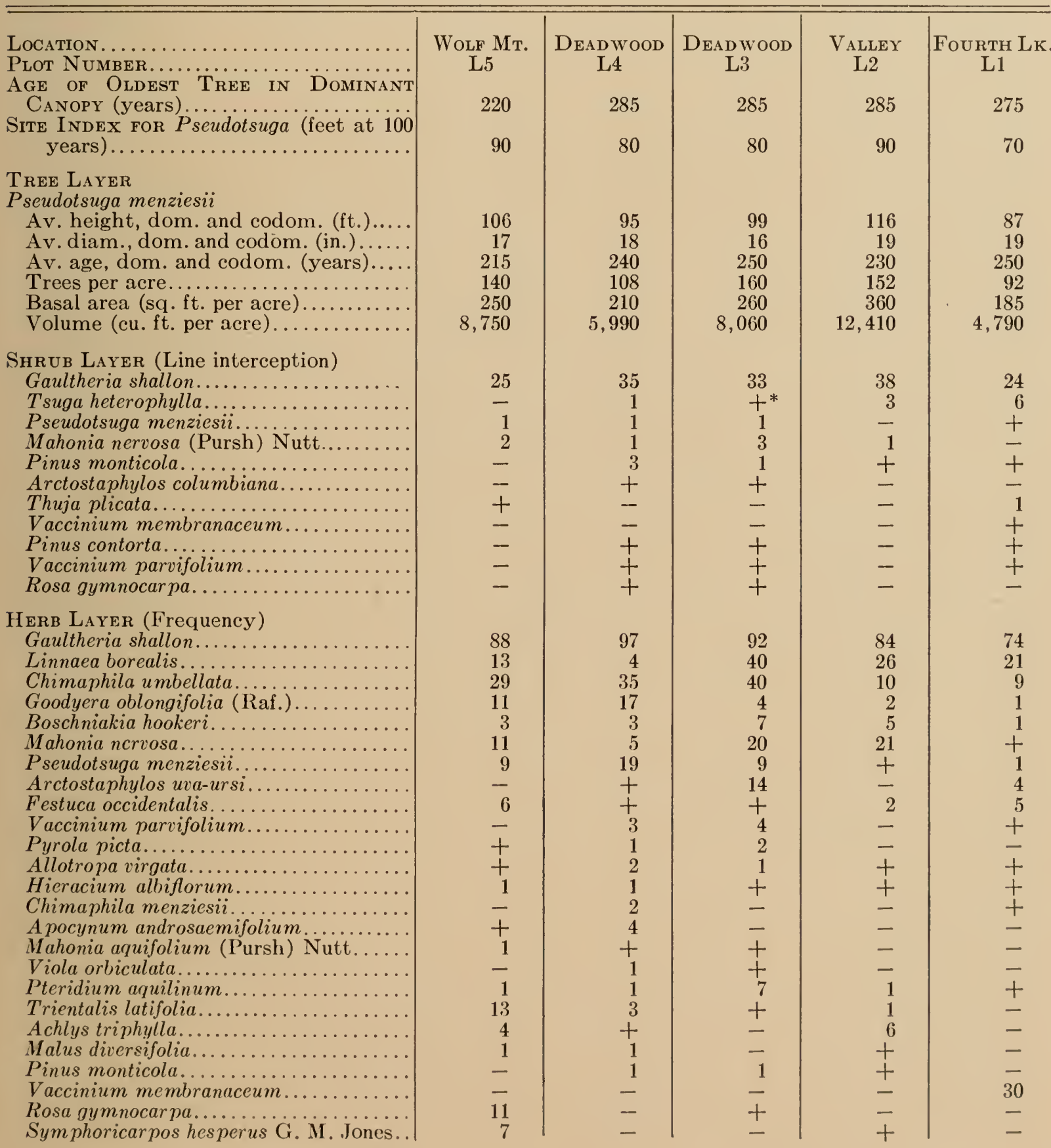


TABLE III-Continued

PSEUDOTSUGA-GAULTHERIA-PELTIGERA ASSOCIATION-Coneluded

Location

Plot Number

Herr LAYEU (Frequency)-Concluded

Campanula scouleri..................

Purola bracteat

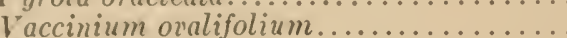

T'suga heterophylla.....

Rubus vitifolius. . .

Bromus vulgaris.

Monotropa latisquamea (Rydb.) Hulten

MOSS-LICHEN LAYER (Point frequency)

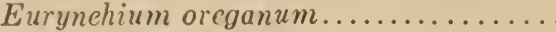

Hyloeomium splendens...............

Rhytidiadel phus triquetrus............

Rhytidiadel phus loreus.................

Dieranum scoparium...

Camptothecium megaptilum Suli.......

Peltigera membranaeea Ach............

Peltigera aphthosa..................

Calliergonella schreberi..............

Dieranum fuseeseens..................

Polytrichum juniperinum.............

Cladonia sylvativa....................

Cladonia graeilis....................

Cladonia squamosa..................

Cladonia fureata..................

Cladonia bellidiflora..................

Cladonia macilenta..................

Cladonia fimbriata....................

Rhaeomitrium lanuginosum............

Rhaeomitrium canescens.............

Rhaeomitrium heterostichum..........

Aulacomnium androgynum............

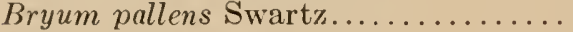

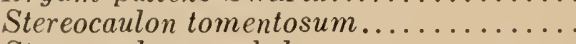

Stereoeaulon pasehale................

Pilophoron eercolus..................

Pseudotsuga menziesii...

On Decaying Wood (Total estimate)

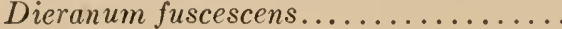

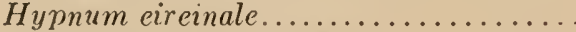

Aulacomnium androgynum

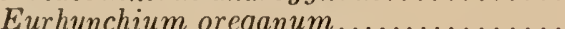

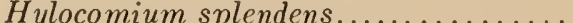

Peltigera aphthosa.......................

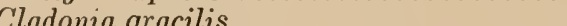

Cladonia bellidiflora ......................

Cladonia fimbriata..........................

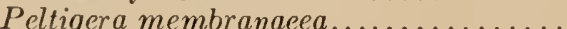

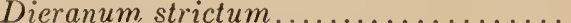

Pseudisotheeium stoloniferum............

Calliergonella sehreberi..................

Polytrichum juniperinum..............

ON Pseudotsuga BARK (Total estimate)

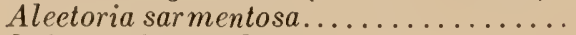

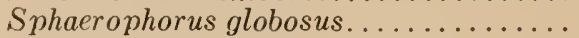

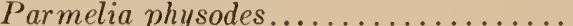

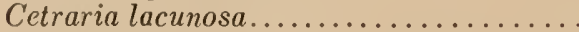

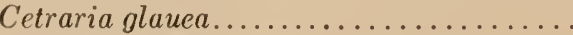

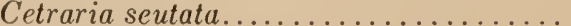

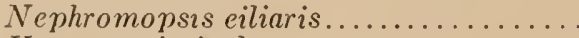

Hypnum eircinale...............

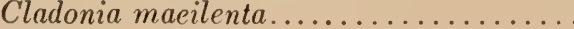

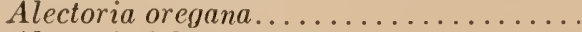

Alectoria jubata.

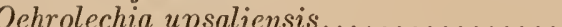

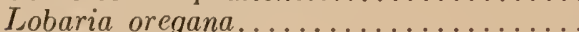

Usnea plicata.

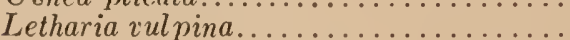

\begin{tabular}{|c|c|c|c|c|}
\hline$\underset{\mathrm{L} .5}{\text { Wor.F. }} \mathrm{MT}$. & $\begin{array}{l}\text { DEADW OOD } \\
\text { L4 }\end{array}$ & $\begin{array}{c}\text { DEABWOOD } \\
\text { L3 }\end{array}$ & $\begin{array}{l}\text { VALLEY } \\
\text { L2 }\end{array}$ & $\begin{array}{c}\text { Fourth LK. } \\
\text { L1 }\end{array}$ \\
\hline 6 & + & + & - & - \\
\hline$\overline{-}$ & $\bar{z}$ & 1 & $\bar{z}$ & $\overline{1}$ \\
\hline$\overline{-}$ & 1 & + & - & $-\frac{1}{1}$ \\
\hline 1 & - & \pm & \pm & \pm \\
\hline+ & + & + & - & $\bar{t}$ \\
\hline 19 & 15 & 23 & 22 & 2 \\
\hline $\begin{array}{l}1 \\
2\end{array}$ & $\begin{array}{r}3 \\
+\end{array}$ & $\begin{array}{r}23 \\
2\end{array}$ & $\begin{array}{l}17 \\
+\end{array}$ & $\begin{array}{r}9 \\
+\end{array}$ \\
\hline- & & + & + & + \\
\hline+ & 5 & 2 & 4 & 2 \\
\hline $\begin{array}{l}2 \\
1\end{array}$ & $\begin{array}{l}1 \\
1\end{array}$ & $\begin{array}{l}1 \\
1\end{array}$ & $\begin{array}{l}3 \\
1\end{array}$ & $\begin{array}{l}1 \\
1\end{array}$ \\
\hline+ & 1 & 1 & + & 1 \\
\hline$+t_{1}$ & + & $\begin{array}{r}9 \\
+\end{array}$ & $\begin{array}{l}3 \\
1\end{array}$ & $\begin{array}{r}8 \\
+\end{array}$ \\
\hline- & 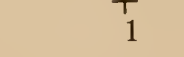 & + & + & + \\
\hline$\overline{-}$ & $\bar{t}$ & $\stackrel{+}{+}$ & $\stackrel{+}{+}$ & $\begin{array}{r}3 \\
+\end{array}$ \\
\hline$\overline{-}$ & - & + & - & + \\
\hline \pm & \pm & $\stackrel{+}{+}$ & \pm & + \\
\hline $\bar{z}$ & - & $t$ & - & - \\
\hline+ & + & $\mp$ & $\bar{I}$ & \pm \\
\hline+ & + & + & + & $\bar{t}$ \\
\hline+ & + & $\dot{+}$ & + & + \\
\hline \pm & + & $\stackrel{+}{+}$ & + & $\bar{z}$ \\
\hline- & + & + & - & - \\
\hline $\bar{t}$ & + & + & $\bar{t}$ & \pm \\
\hline+ & + & + & + & + \\
\hline 1 & + & 1 & 3 & 1 \\
\hline $\bar{t}$ & $\bar{t}$ & $\bar{t}$ & $\begin{array}{l}3 \\
2 \\
2\end{array}$ & $\bar{z}$ \\
\hline+ & + & 1 & 3 & 2 \\
\hline $\bar{t}$ & $\bar{t}$ & \pm & $\begin{array}{l}3 \\
2 \\
2\end{array}$ & + \\
\hline+ & + & + & 2 & 1 \\
\hline 1 & 1 & - & $\mp$ & \pm \\
\hline- & + & + & + & + \\
\hline+ & + & + & + & + \\
\hline - & $\overline{-}$ & $\bar{t}$ & 1 & + \\
\hline+ & + & - & - & - \\
\hline 3 & 4 & 3 & 1 & 5 \\
\hline 2 & 1 & $\begin{array}{l}1 \\
2\end{array}$ & $\begin{array}{l}3 \\
2\end{array}$ & $\begin{array}{l}3 \\
1\end{array}$ \\
\hline 2 & 3 & 2 & 2 & - \\
\hline- & - & 3 & - & 3 \\
\hline 1 & $\begin{array}{l}1 \\
0\end{array}$ & 1 & $\overline{2}$ & $\bar{z}$ \\
\hline $\begin{array}{l}2 \\
1\end{array}$ & + & 1 & 2 & 2 \\
\hline- & 3 & 2 & 2 & \\
\hline $\bar{t}$ & $\bar{t}$ & 1 & $\bar{z}$ & $\begin{array}{l}2 \\
2\end{array}$ \\
\hline & 1 & 1 & - & - \\
\hline - & $\bar{t}$ & + & \pm & $=$ \\
\hline+ & - & + & - & - \\
\hline
\end{tabular}


$\overline{\text { Location } \ldots \ldots \ldots \ldots \ldots \ldots \ldots \ldots \ldots \ldots \ldots \ldots \ldots \ldots \ldots \ldots \ldots \ldots}$

Plot Number.

Age of Oldest Tree in Dominant CANopy

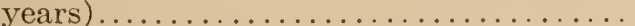

Site INDEX FOR Pseudotsuga (feet at 100 years).

Tree Layer

Pseudotsuga menziesii

Av. height, dom. and codom. (ft.).........

Av. diam., dom. and codom. (in.)..........

Av. age, dom. and codom. (years).

Tree per acre..

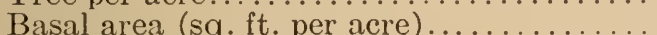

Volume (cu. ft. per acre)...

Wolf
G5
2
130




2
10
300

Tsuga heterophylla

Av. height, dom. and codom. (ft.)........

Av. diam., dom. and codom. (in.)...........

Av. age, dom. and codom. (years)........

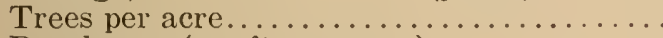

Basal area (sq. ft. per acre).

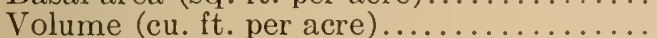

Thuja plicata

Av. height, dom. and codom. (ft.)........

Av. diam., dom. and codom. (in.)..........

Av. age, dom. and codom. (years)

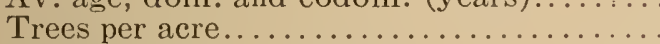

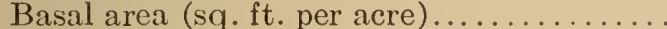

Volume (cu. ft. per acre).

Shrub Layer (Line interception)

Gaultheria shallon.

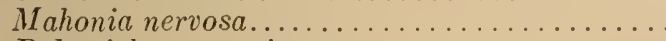

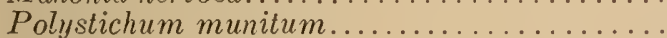

Vaccinium parvifolium.................

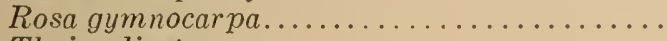

Thuja plicata.

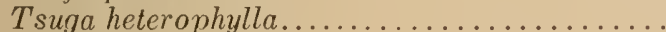

Pseudotsuga menziesii.

Holodiscus discolor.

Herb Layer (Frequency)

Gaultheria shallon. ...

Linnaea borealis . ................

Chimaphila umbellata..................

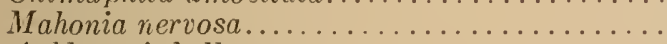

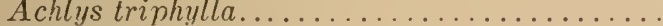

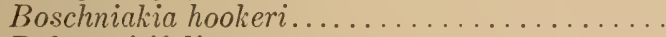

Rubus vitifolius.

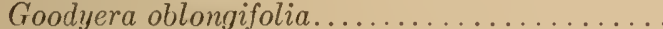

Rosa gymnocarpa.

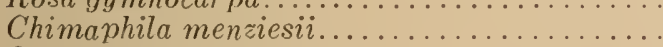

Symphoricarpos hesperus...............

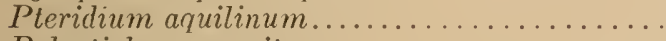

Polystichum munitum.....................

Vaccinium parvifolium.

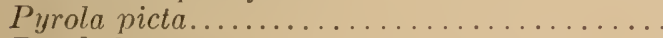

Pyrola bracteata.

Moss-Lichen LAyer (Point frequency)

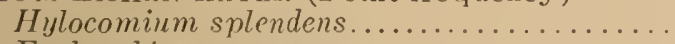

Eurh!nchium oreganum.................

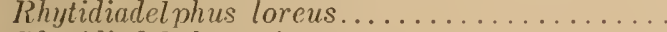

Rhytidiadcl phus triquetrus....

Camptothecium megaptilum..............

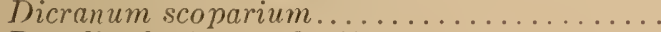

Pseudisothecium stoloniferum

Hypnum circinale.

On Decayng Wood (Total Estimate)

Hylocomium splendens.

Eurhynchium oreganum.

\begin{tabular}{l|r|r|r} 
MT. & $\begin{array}{c}\text { Deadwood } \\
\text { G4 }\end{array}$ & $\begin{array}{c}\text { Dead G6 } \\
\text { G6 }\end{array}$ & $\begin{array}{c}\text { Valley } \\
\text { G3 }\end{array}$ \\
220 & 320 & 320 & \\
130 & 140 & 120 & 275 \\
& & & 140 \\
& & & \\
147 & 175 & 148 & 169 \\
22 & 27 & 26 & 28 \\
215 & 300 & 300 & 250 \\
100 & 108 & 104 & 64 \\
300 & 470 & 410 & 280 \\
320 & 23,650 & 17,880 & 12,840 \\
& & &
\end{tabular}

\begin{tabular}{|c|c|c|}
\hline- & - & 66 \\
\hline- & - & 10 \\
\hline- & - & 190 \\
\hline - & - & 40 \\
\hline- & - & 24 \\
\hline & - & 640 \\
\hline
\end{tabular}

60

11

130
12

7

135

31

$+$

$-$ 
TABLE III-Continued

\section{PSEUDOTSUGA-GAULTHERIA ASSOCIATION-Concluded}

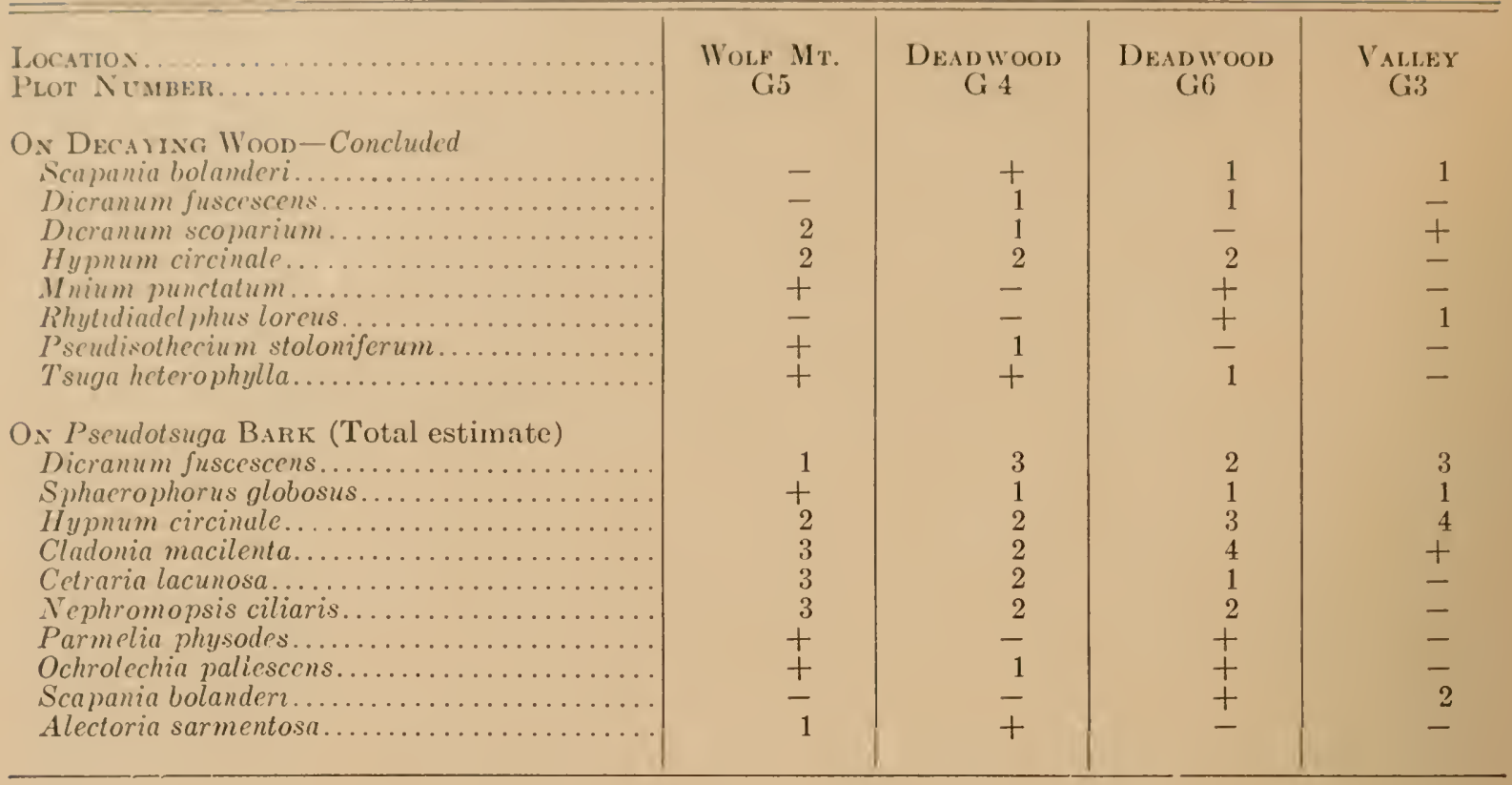

\section{PSEUDOTSUGA-TSUGA-GAULTHERIA ASSOCIATION}

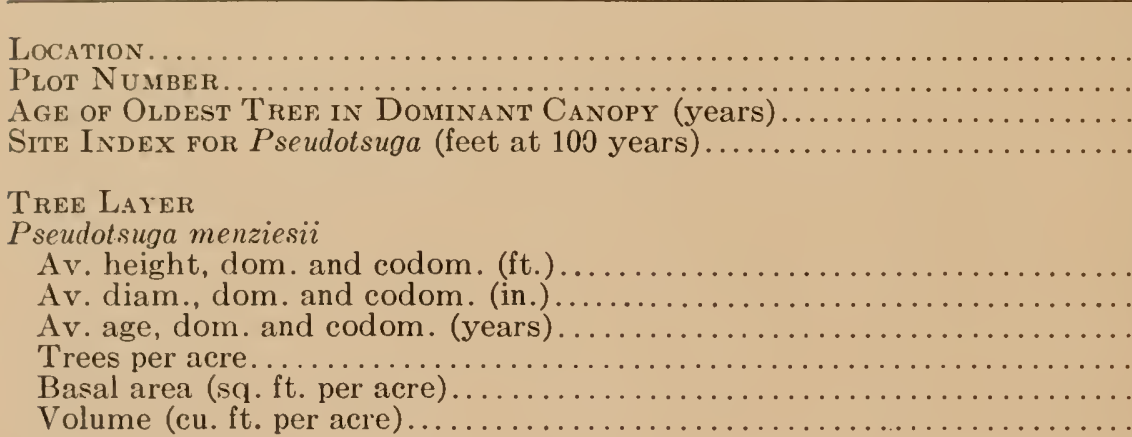

\begin{tabular}{|c|c} 
Fourth LK. & Echo MT. \\
G1 2 \\
245 & 285 \\
90 & 60 \\
& \\
& \\
110 & 77 \\
17 & 14 \\
235 & 260 \\
172 & 104 \\
300 & 130 \\
10,310 & 2,950
\end{tabular}

Tsuga heterophylla

Av. height, dom. and codom. (ft.).

Av. diam., dom. and codom. (in.).

Av. age, dom. and codom. (years).

Trees per acre.

Basal area (sq. fit. per acre)

Volume (cu. ft. per acre).

Thuja plicata

Av. height, dom. and codom. (ft ).

Av. diam., dom and codom. (in.).

Av. age, dom. and codom. (years).

Trees per acre.

Basal area (sq. ft. per acre)

Volume (cu. ft. per acre).

\section{Shrub Layer (Line interception)}

Gaultheria shallon.

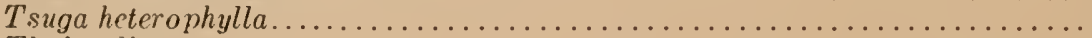

Thuja plicata.

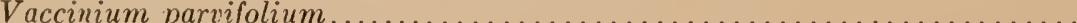

Vaccinium membranaceum.

Herb Layer (Frequency)

Gaultheria shallon.

Linnaea borealis

Vaccinium parvifolium

Goodyera oblongifolia.

\begin{tabular}{r|r}
71 & 70 \\
10 & 12 \\
160 & 250 \\
32 & 120 \\
17 & 120 \\
490 & 2,540 \\
& \\
74 & 64 \\
14 & 9 \\
200 & 230 \\
36 & 24 \\
44 & 12 \\
1,190 & 325 \\
& \\
50 & 36 \\
+ & + \\
+ & + \\
- & + \\
- & + \\
& \\
76 & 56 \\
50 & 5 \\
12 & 5 \\
6 &
\end{tabular}




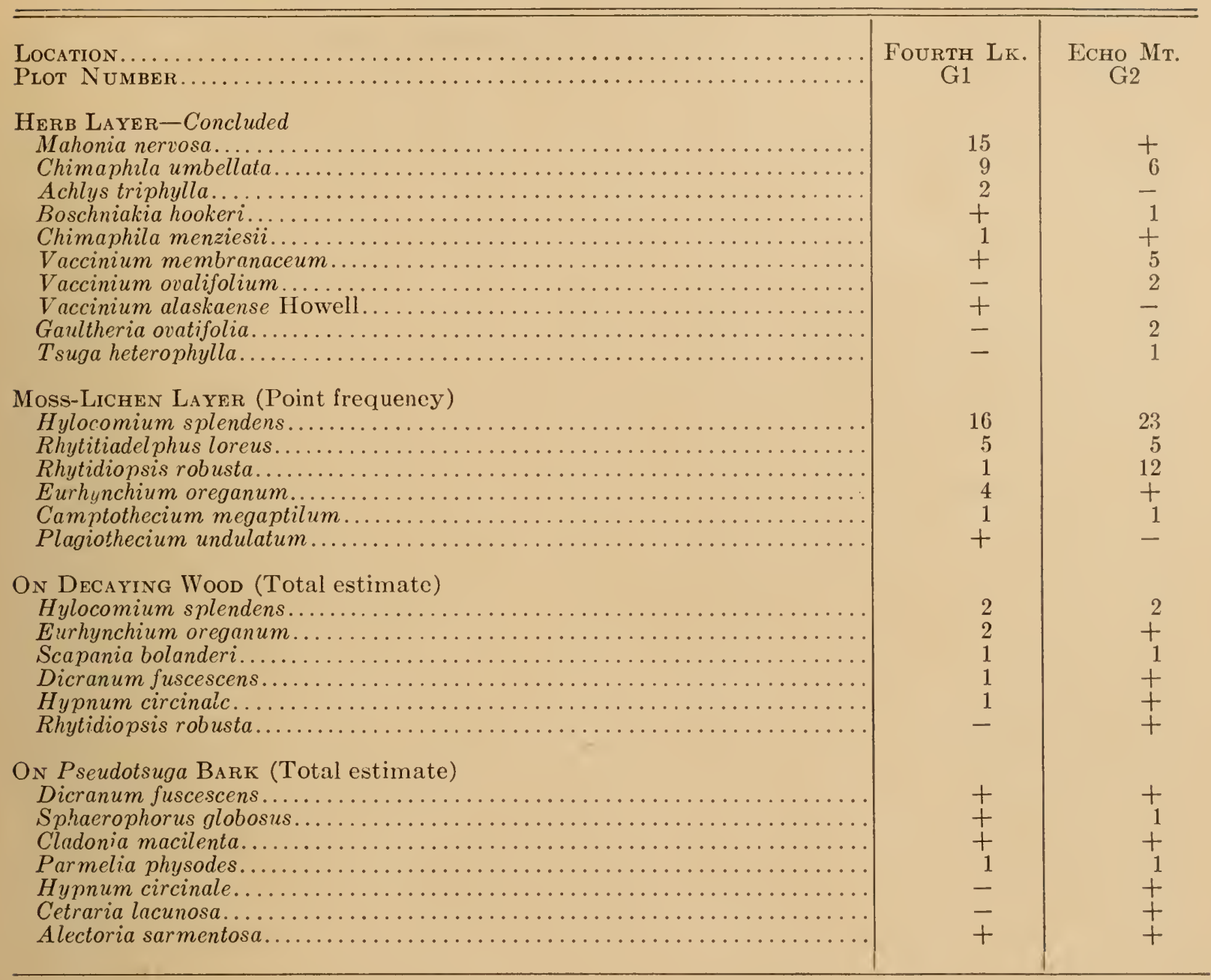

\section{PSEUDOTSUGA-TSUGA-HYLOCOMIUM ASSOCIATION}

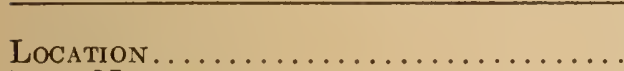

Plot Number.

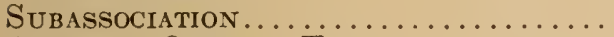

Age OF OLdest TReE IN DOMINANT CANOPY (years) ...........................

Site Index for Pseudotsuga (feet at 100 years)..

\begin{tabular}{|c|r|}
$\begin{array}{c}\text { DeADWOOD } \\
\text { M4 } \\
\text { typicum }\end{array}$ & $\begin{array}{c}\text { VALLEY } \\
\text { M3 } \\
\text { typicum }\end{array}$ \\
& \\
320 & 320 \\
130 & 130 \\
& \\
& \\
158 & 157 \\
26 & 23 \\
290 & 300 \\
96 & 84 \\
380 & 270 \\
140 & 12,870 \\
& \\
85 & 86 \\
9 & 10 \\
190 & 190 \\
176 & 88 \\
92 & 50 \\
3,670 & 1,710 \\
& \\
101 & 78
\end{tabular}

Tree Layer

Pseudotsuga mcnziesii

Av. height, dom. and codom. (ft.)... Av. diam., dom. and codom. (in.)..... Av. age, dom. and codom. (years)....

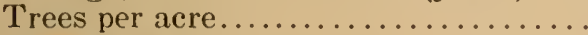
Basal area (sq. ft. per acre).......... Volume (cu. ft. per acre).

Tsuga heterophylla

Av. height, dom. and codom. (ft.)...

Av. diam., dom. and codom. (in.).... Av. age, dom. and codom. (years).... Trees per acre.

Basal area (sq. ft. per acre).......... Volume (cu. ft. per acre).

Thuja plicata

Av. height, dom. and codom. (ft.)....

\begin{tabular}{|c|r|r}
$\begin{array}{c}\text { FourTh LK. } \\
\text { M1 }\end{array}$ & $\begin{array}{c}\text { WolF MT. } \\
\text { M5 } \\
\text { typicum }\end{array}$ & $\begin{array}{c}\text { Echo MT. } \\
\text { nu2 }\end{array}$ \\
& nudum \\
275 & 220 & 285 \\
120 & 170 & 170 \\
& & \\
& & \\
144 & 199 & 209 \\
22 & 33 & 37 \\
260 & 210 & 250 \\
68 & 80 & 72 \\
210 & 500 & 550 \\
9,110 & 27,960 & 31,400 \\
& & \\
136 & 65 & 87 \\
18 & 7 & 10 \\
220 & 80 & 170 \\
64 & 96 & 52 \\
140 & 26 & 28 \\
730 & 750 & 960 \\
& & \\
85 & - & 107
\end{tabular}


T.ABILE III-Continued

PSEC DOTSLGATSUGA-HYLOCOMIU.M ASSOCIATION-Concluded

I.OC.iTION.

l'LOT N

Thuju micula-concluded

$\Lambda$ r. diam., dom. and codom. (in.)..

Av. age, dom. and codom. (years)..

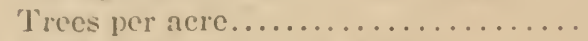

Basal area (sq. ft. per acre).

Volume (cu. ft. per acre)..........

SHrub LAyer (Line interception)

Gaultheria shallon..................

Mahonia nerrosa...................

Polystichum munitum................

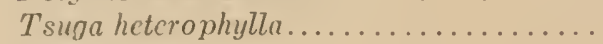

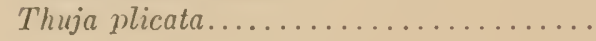

Herb Lajer (Frequency)

Chimaphila menziesii..............

Mahonia ncrvosa.................

Linnaea borcalis...................

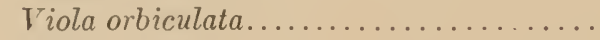

Pyiola picta.......................

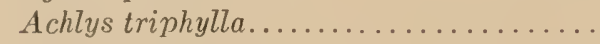

Gaulthcria shallon..................

Goodycra oblongifolia...............

Vaccinium parvifolium...............

Chimaphila umbellata..............

Monotropa uniflora..................

Monotropa latisquamea...............

Listcra cordata.....................

Corallorhiza maculata................

Polystichum munitum................

Trillium ovatum......

Moss-Lichen Layer (Point frequency)

Eurhynchium orcganum.

Hylocomium splendens....

Rhytidiadclphus lorcus......

Rhytidiadelphus triquetrus.

Pseudisothecium stolonifer um ............

Camptothecium megaptilum...........

Dicranum fuscescens................

Mnium spinulosum...............

On Decaying Wood (Total estimate).

Hypnum circinale..................

Cephalozia media..................

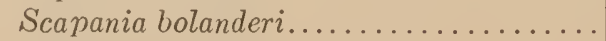

Dicranum fuscescens................

Lepidozia reptans....................

Rhytidiadelphus loreus...............

Eurhynchium oreganum..............

Hylocomium splendens...............

Tsuga heterophylla.

On Pseudotsuga Bark (Total estimate)

Hynum circiralc...................

Dicranum fuscescens.................

Cladonia macilenta..

Scapania bolandcri.

\begin{tabular}{|c|c|c|c|c|}
\hline $\begin{array}{c}\text { DEA1)WOO1) } \\
\text { M4 }\end{array}$ & $\begin{array}{l}\text { VALLLEY } \\
M 33\end{array}$ & $\begin{array}{c}\text { FourTh LK. } \\
\text { ग11 }\end{array}$ & $\begin{array}{c}\text { Woly MT. } \\
\text { MI }\end{array}$ & $\begin{array}{c}\text { Echo } 11 \mathrm{~T} . \\
\mathrm{N} 2\end{array}$ \\
\hline 13 & 13 & 14 & - & 14 \\
\hline 190 & 200 & 220 & - & 170 \\
\hline 12 & 12 & 56 & - & 16 \\
\hline 13 & 11 & 68 & - & 20 \\
\hline 500 & 350 & 2,260 & - & 800 \\
\hline 5 & + & 3 & + & + \\
\hline 1 & - & 2 & - & - \\
\hline 3 & + & + & + & + \\
\hline 1 & + & + & + & + \\
\hline- & - & + & - & + \\
\hline 7 & 2 & 2 & + & 12 \\
\hline 11 & + & 9 & 1 & 1 \\
\hline 2 & 11 & 49 & + & - \\
\hline 1 & - & 1 & 1 & 6 \\
\hline 1 & + & 1 & + & 1 \\
\hline 3 & 3 & 4 & - & 3 \\
\hline 13 & 1 & 19 & + & + \\
\hline 1 & + & + & 2 & 1 \\
\hline+ & 1 & 21 & + & + \\
\hline+ & 2 & 12 & + & + \\
\hline+ & $\begin{array}{l}+ \\
+\end{array}$ & \pm & \pm & $\bar{t}$ \\
\hline$\overline{-}$ & \pm & $\overline{-}$ & $\bar{t}$ & + \\
\hline- & + & - & + & - \\
\hline+ & + & - & + & + \\
\hline+ & - & + & + & + \\
\hline 10 & 18 & 17 & 3 & 2 \\
\hline 44 & 35 & 8 & 2 & + \\
\hline+ & 8 & 2 & + & + \\
\hline+ & 1 & - & + & - \\
\hline- & - & 2 & - & + \\
\hline$\overline{-}$ & + & $+_{2}$ & $\begin{array}{r}1 \\
+\end{array}$ & \pm \\
\hline $\bar{t}$ & - & ${ }_{-}^{2}$ & + & + \\
\hline- & - & - & + & + \\
\hline 4 & 4 & 3 & 4 & 4 \\
\hline 2 & 1 & 2 & 2 & 2 \\
\hline 2 & 1 & - & 2 & 1 \\
\hline 2 & 1 & 1 & 2 & 1 \\
\hline 1 & - & - & 1 & 1 \\
\hline+ & + & + & + & - \\
\hline $\begin{array}{l}2 \\
2\end{array}$ & + & + & 1 & $\overline{-}$ \\
\hline $\begin{array}{l}2 \\
2\end{array}$ & $\begin{array}{l}1 \\
+\end{array}$ & $\begin{array}{l}1 \\
1\end{array}$ & $\begin{array}{l}1 \\
1\end{array}$ & $\overline{1}$ \\
\hline & & & & \\
\hline $\begin{array}{l}4 \\
?\end{array}$ & $\begin{array}{l}3 \\
3\end{array}$ & $\begin{array}{l}2 \\
?\end{array}$ & 4 & $\begin{array}{l}2 \\
2\end{array}$ \\
\hline $\begin{array}{l}2 \\
3\end{array}$ & $\begin{array}{l}3 \\
2\end{array}$ & -2 & $\begin{array}{l}1 \\
4\end{array}$ & - \\
\hline- & 1 & - & + & - \\
\hline
\end{tabular}


TABLE III-Continued PSEUDOTSUGA-POLYSTICHUM ASSOCIATION

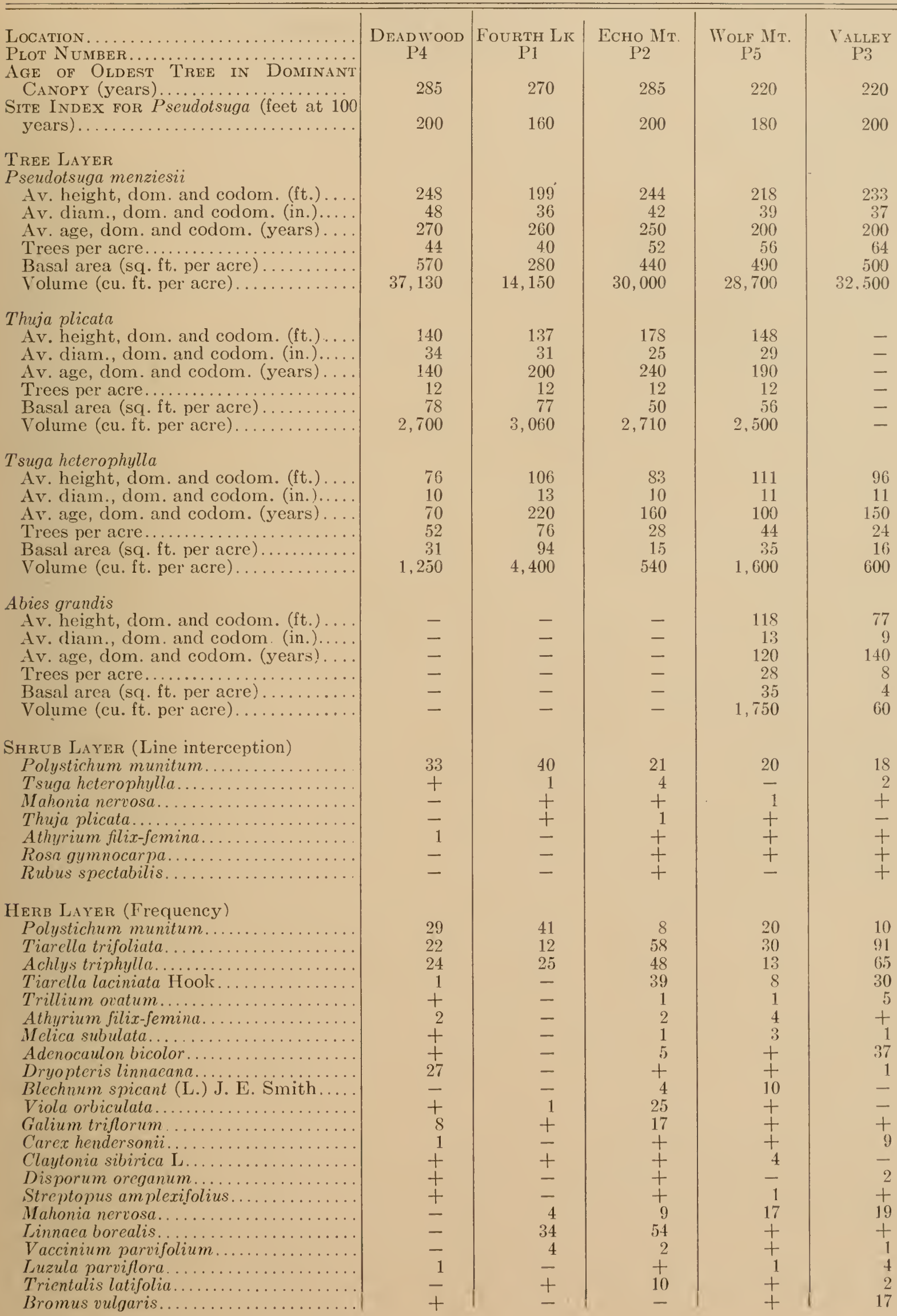


TABLE III-Continued

PSEUDOTSUGA-POLYSTICHUM ASSOCIATION-Concluded

Location

Plot NuMBer

Moss-LicheN LAYER (Point Frequeney) Eurhynchium oreganum...

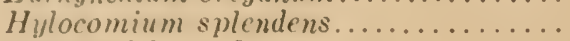
Rhytidiadel phus loreus................... Mnium insigne.

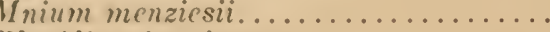

Rhytidiopsis robusta...............

On Decaring Wood (Total estimate) Le pidozia reptans.

Mnium punctatum.

Inium spinulosum.

Eurhynchium oreganum .............

Hylocomium splendens..............

Rhytidiadel phus loreus................

Pseudisothecium stoloniferum............

Cephalozia media. .

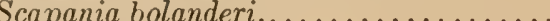

Icmadophila ericetorum................. Riccardia latifrons. .

Tsuga heterophylla...

ON Pseudotsuga BARK (Total estimate)

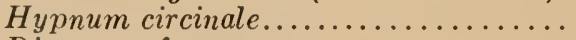

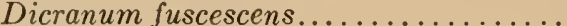

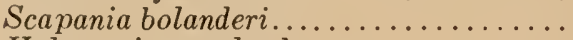

Hylocomium splendens..............

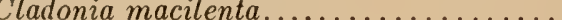

Sphaerophorus globosus.................

ON Tsuga BARK (Total estimate)

Pseudisothecium stoloniferum.

Neckera douglasii...................

Frullania nisquallensis.

Hypnum circinale..................

Dicranum fuscescens.................

Eurhynchium oreganum

Cladonia macilenta.

Rhytidiadelphus loreus...............

\begin{tabular}{|c|c|c|c|c|}
\hline $\begin{array}{c}\text { DEA1) WOOD } \\
\text { P4 }\end{array}$ & $\underset{\text { POURT LK. }}{\text { Fourt }}$ & $\underset{\text { Еса }}{\text { Ест. }}$ & $\underset{155}{\text { Wolf } M T .}$ & $\begin{array}{c}\text { VALLEY } \\
\text { I'3 }\end{array}$ \\
\hline 2 & 9 & 3 & 8 & 9 \\
\hline 10 & + & + & 2 & 1 \\
\hline- & + & 1 & - & + \\
\hline 4 & - & - & 1 & 3 \\
\hline- & - & + & 1 & - \\
\hline- & + & + & - & - \\
\hline 1 & 1 & 2 & 2 & 1 \\
\hline+ & + & 1 & 2 & 1 \\
\hline+ & + & + & + & + \\
\hline 1 & - & + & + & - \\
\hline 3 & - & 2 & 3 & 2 \\
\hline 2 & 2 & 3 & 4 & 3 \\
\hline 1 & + & 1 & 2 & 1 \\
\hline 2 & 1 & 1 & + & 2 \\
\hline 1 & - & 2 & 4 & 2 \\
\hline+ & + & 1 & 1 & 1 \\
\hline 2 & 1 & 1 & 2 & 1 \\
\hline- & + & - & + & - \\
\hline 1 & - & - & 1 & - \\
\hline 1 & 1 & - & 3 & 1 \\
\hline- & 2 & 3 & + & + \\
\hline 2 & 3 & - & 4 & 4 \\
\hline 1 & 3 & 2 & 3 & 3 \\
\hline- & 3 & 2 & 2 & 2 \\
\hline 1 & - & 2 & - & 1 \\
\hline- & 3 & 2 & 6 & 3 \\
\hline- & + & - & - & + \\
\hline 2 & 2 & 3 & 3 & 4 \\
\hline 1 & 1 & 2 & - & 2 \\
\hline- & 1 & 1 & - & 1 \\
\hline 2 & 1 & - & 1 & 2 \\
\hline- & 1 & - & - & 1 \\
\hline- & 1 & 1 & 4 & + \\
\hline 1 & 1 & 2 & 1 & + \\
\hline 1 & - & - & 1 & 1 \\
\hline 1 & 1 & - & 2 & 1 \\
\hline- & - & + & - & + \\
\hline
\end{tabular}

THUJA-LYSICHITUM ASSOCIATION

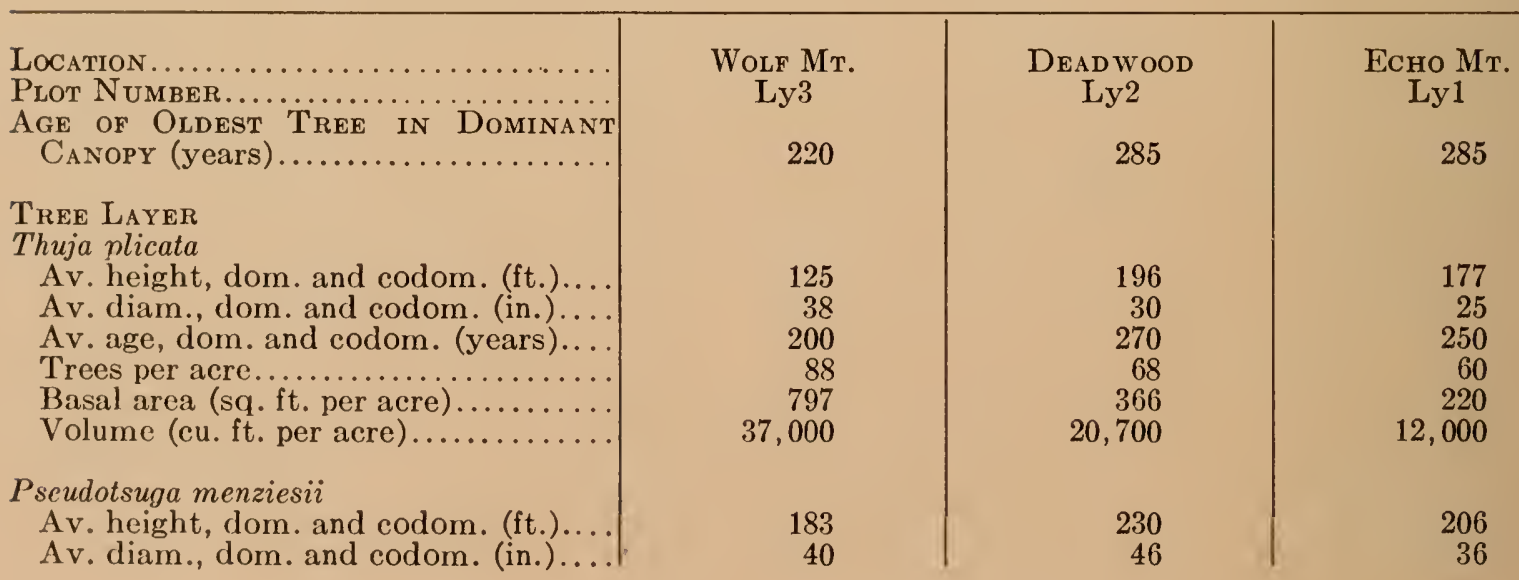


THUJA-LYSICHITUM ASSOCIATION-Continued

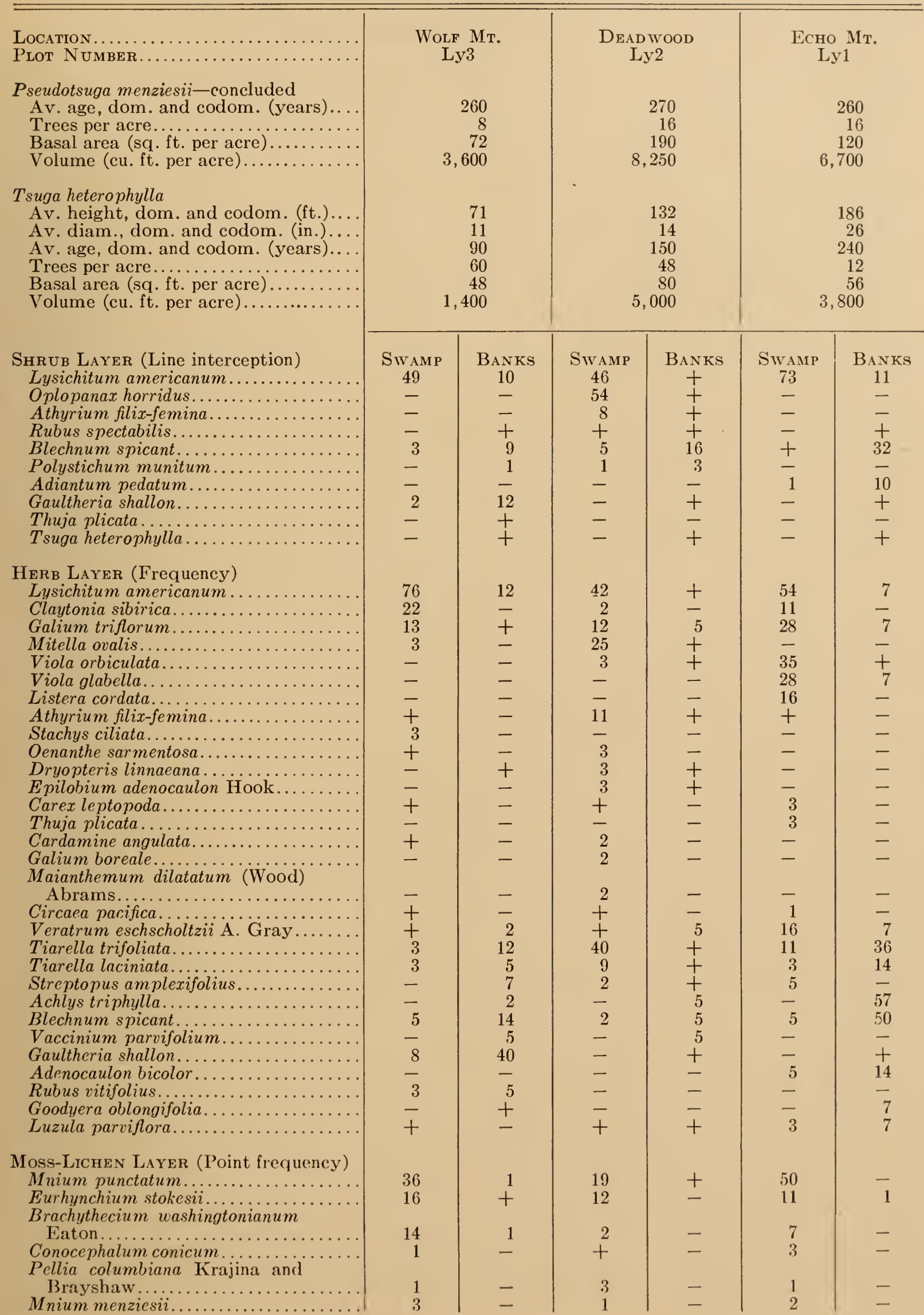


TABLE III-Concluded

THUJA-L YSICHITUM ASSOCIATION-Concluded

\begin{tabular}{|c|c|c|c|c|c|c|}
\hline 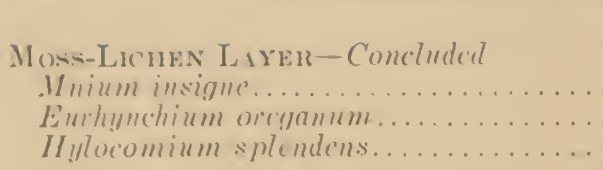 & $\begin{array}{l}\text { SiNAMP } \\
+ \\
+ \\
-\end{array}$ & $\begin{array}{l}\text { BANKS } \\
\begin{array}{r}-10 \\
5\end{array}\end{array}$ & $\begin{array}{l}\text { SWAMP } \\
2 \\
+ \\
+\end{array}$ & $\begin{array}{l}\text { BANKA } \\
\qquad \begin{array}{l}- \\
2\end{array}\end{array}$ & $\begin{array}{l}\text { SWAMP } \\
+ \\
+ \\
-\end{array}$ & $\begin{array}{l}\text { BANKS } \\
- \\
\overline{+}\end{array}$ \\
\hline 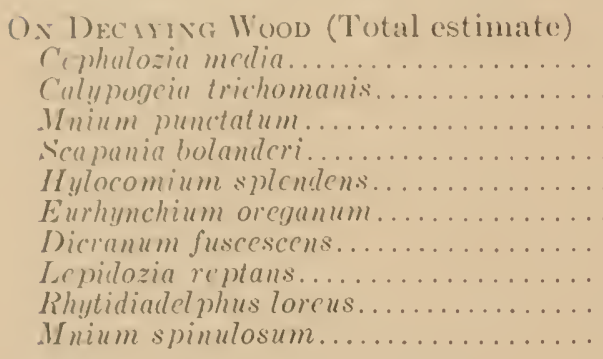 & & $\begin{array}{r}3 \\
2 \\
2 \\
3 \\
4 \\
3 \\
+ \\
2 \\
1 \\
+ \\
+\end{array}$ & & $\begin{array}{l}2 \\
+ \\
1 \\
1 \\
2 \\
2 \\
1 \\
1 \\
1 \\
+\end{array}$ & & $\begin{array}{r}2 \\
+ \\
2 \\
2 \\
1 \\
1 \\
2 \\
1 \\
1 \\
+\end{array}$ \\
\hline 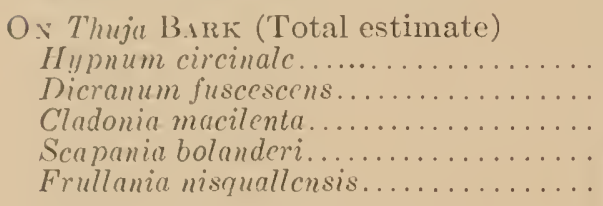 & & $\begin{array}{l}1 \\
+ \\
1 \\
1 \\
-\end{array}$ & & $\begin{array}{l}2 \\
2 \\
1 \\
+ \\
+\end{array}$ & & $\begin{array}{l}3 \\
2 \\
3 \\
+ \\
+\end{array}$ \\
\hline
\end{tabular}

* Less than one percent.

** According to the total estimate scale of Krajina and Domin (19). Interpretation of the scale values as follows:

+ solitary, with small dominance.

1 seldom, with small dominance.

2 very scattered, with small dominance

3 scattered, with small dominance.

4 often, with $1 / 20$ dominance.

5 of ten, with $1 / 5$ dominance.

6 any number, with $1 / 4$ to $1 / 3$ dominance.

7 any number, with $1 / 3$ to $1 / 2$ dominance.

8 any number, with $1 / 2$ to $3 / 4$ dominance.

9 any number, with dominance more than $3 / 4$, but less than complete.

10 any number, with complete dominance.

\section{Pseudotsuga-Tsuga-Gaultheria association}

Site index for Pseudotsuga menziesii in the Pseudotsuga-Tsuga-Gaultheria plots was as low as in the Pseudotsuga-Gaultheria-Peltigera plots (Table III). Unlike the Pseudotsuga-Gaultheria-Peltigera and Pseudotsuga-Gaultheria plots, Tsuga heterophylla constituted nearly half the stand volume of the Echo Mountain Pseudostuga-Tsuga-Gaultheria Plot, although little occurred in the Fourth Lake Plot. Tsuga heterophylla heights and diameters were poor, nevertheless (Fig. 7).

The presence of Chamaecyparis nootkatensis, Vaccinium membranaceum, $V$. alaskaense, and Gaultheria ovatifolia showed the affinity of the PseudotsugaTsuga-Gaultheria plots with stands characteristic of relatively high altitudes. Other plants of shrub and herb layers occurred in both the Pseudotsuga-Gaultheria and Pseudotsuga-Tsuga-Gaultheria plots, although many were smaller and less abundant in the Pseudotsuga-Tsuga-Gaultheria plots than they were in Pseudotsuga-Gaultheria sites at lower elevations. In the moss-lichen layer, Rhytidiadelphus loreus and Rhytidiopsis robusta were other species common in high altitude stands, although the predominant moss, Hylocomium splendens, was equally widespread in lower altitude plots. Both mosses and lichens were common on the bark of trees, with Lobaria oregana being particularly conspicuous on both living and dead branches in the Echo Mountain Plot. 
The average height of 144 to $155 \mathrm{ft}$. for the dominant and codominant Pseudotsuga menziesii in the subassociation typicum plots was considerably less than the 199 to $209 \mathrm{ft}$. averaged in the nudum plots (Table III). In most plots Tsuga heterophylla formed a secondary canopy 70 to $130 \mathrm{ft}$. below the dominant Pseudotsuga menziesii canopy. Although as numerous as Pseudotsuga menziesii, Tsuga heterophylla made up only a minor proportion of stand volume because individual trees were smaller. With the exception of the Fourth Lake Plot, however, all Tsuga heterophylla were younger than the trees of the dominant canopy. In the Fourth Lake Plot, some Tsuga heterophylla were of the same age class as the Pseudotsuga menziesii and both species were represented in the codominant canopy (Fig. 8).

The subordinate vegetation of the Pseudotsuga-Tsuga-Hylocomium plots was characterized by an almost complete lack of shrubs and herbs. Various inconspicuous plants characteristic of the association were present, but their cover value was small (Table III). Stunted Gaultheria shallon, Polystichum munitum, and Achlys triphylla, species characteristic of other associations, also occurred locally, but their frequency was low.

The forest floor in the subassociation typicum plots consisted of an almost continuous green carpet of mosses (Fig. 9), Hylocomium splendens and Eurhynchium oreganum being the predominant species. The forest floor in the subassociation nudum plots was essentially bare (Fig. 10). Epiphytic lichens were absent from the lower boles of trees in all plots, but bryophytes such as Hypnum circinale, Dicranum fuscescens, and Scapania bolanderi were quite common.

\section{Pseudotsuga-Polystichum association}

The largest trees and highest volumes per acre of any stand were recorded in the Pseudotsuga-Polystichum plots (Table III). Tree heights on the Fourth Lake Plot (P1) were somewhat lower than average for the association because this plot was located toward the upper margin of the stand type. A logging setting lower down the same slope, which had been cut prior to the present study, had large stump diameters and a high site index comparable with those of the other plots of this association (22). With the exception of the Valley Plot (P3), Thuja plicata was the tallest tree of the secondary canopy, although its frequency was low. Tsuga heterophylla constituted the bulk of a secondary canopy, some 100 to $150 \mathrm{ft}$. below the crowns of the dominants. Tsuga heterophylla volumes were low in most plots because trees were small and considerably younger than the Pseudotsuga menziesii of the dominant canopy.

Polystichum munitum clumps, commonly $80 \mathrm{~cm}$. high, dominated subordinate plant layers (Fig. 11). Shrubby plants had little cover value. On some plots other ferns, such as Blechnum spicant on Plot P5, Dryopteris linnaeana on Plot P4, and Athyrium felix-femina on Plot P2, were also common. Achlys triphylla, a characteristic herb of the Pseudotsuga-Polystichum association, was particularly common in the Valley Plot (P3). This plot represented a subtype in which Achlys triphylla is even more abundant than Polystichum munitum (20). Other herbs characteristic of moist and fertile soils, such as Tiarella trifoliata, Trillium ovatum, Adenocaulon bicolor, and Galium triflorum, were represented on most plots. Mnium insigne and M. menziesii, mosses characteristic of the Pseudotsuga-Polystichum association, were present in the moss-lichen layer and other characteristic bryophytes occurred on decaying wood. Epiphytic mosses were also quite abundant, another feature typical of PseudotsugaPolystichum stands. 


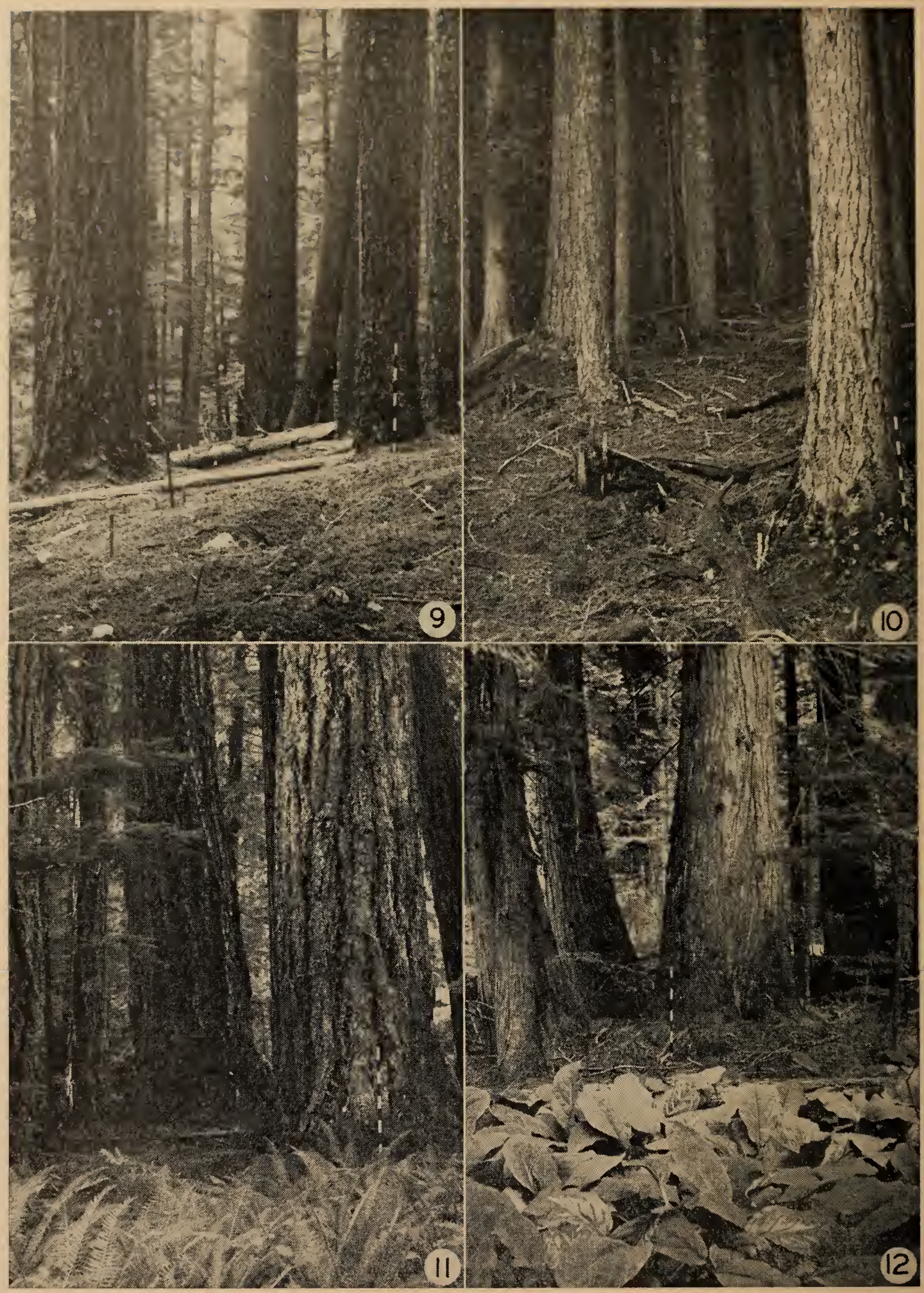

Figure $9-\mathrm{A}$ stand of the Pseudotsuga-Tsuga-Hylocomium association, showing the mossy carpet present in subassociation typicum stands.

Figure 10-A stand of the Pseudotsuga-Tsuga-Hylocomium association, showing the bare forest floor of subassociation nudum stands.

FIGURE 11-A stand of the Pseudotsuga-Polystichum association. The large diameters of Pseudotsuga menziesii are evident.

FIGURE 12-A stand of the Thuja-Lysichitum association. The Lysichitum americanum leaves in the foreground cover a swampy portion of the stand. The Thuja plicata behind are growing on a marginal hummock. 


\section{Thuja-Lysichitum association}

Although trees were restricted to the hummocks and banks which bordered swampy areas, volumes per acre in the Thuja-Lysichitum plots were quite high because individual Thuja plicata trees were large (Table III). The occasional Pseudotsuga menziesii included in these plots more properly belonged to adjacent sites bordering the stands analyzed.

Lysichitum americanum dominated swampy areas in the Thuja-Lysichitum plots during the growing season (Fig. 12). Other species characteristic of the association which grew among and beneath the Lysichitum americanum leaves included Veratrum eschscholtzii, Mitella ovalis, Circaea pacifica, Mnium punctatum, and Conacephalum conicum. Hummocks and the marginal banks of swamps supported a variety of species which also occurred in other associations.

\section{Microclimate}

\section{Precipitation Interception}

During the summer, average interception by the tree canopy ranged from 30 to 57 per cent (Table IV). Presumably such high values were recorded because summer rainfall occurred mostly as light showers, and a large proportion of each shower was required to wet the tree canopy before penetration took place. Low humidities and high temperatures might then evaporate much of this foliage moisture before it could drop to the ground (15). Interception by the tree canopy undoubtedly substantially reduced the amount of water that summer rainfall could add to soil-moisture reserves.

TABLE IV

AVERAGE PERCENTAGE OF PRECIPITATION INTERCEPTED BY THE TREE CANOPIES OF PLOTS REPRESENTATIVE OF FOREST ASSOCIATIONS IN THE NANAIMO RIVER VALLEY, BRITISH COLUMBIA, 1951-1953

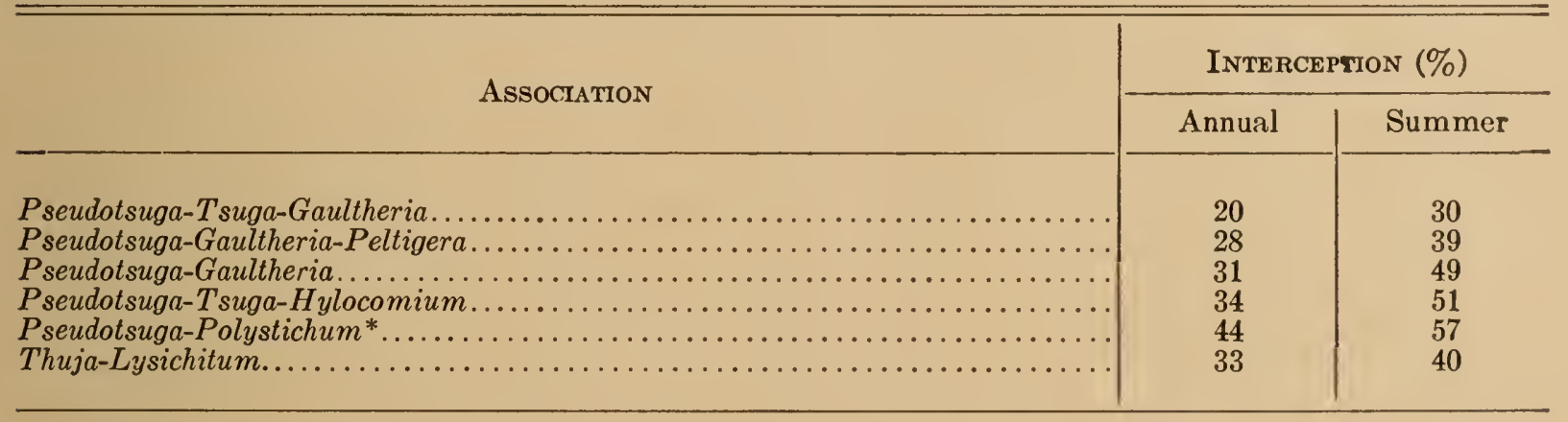

*Excluding Plots P1 and P2, which were understocked.

During periods of high rainfall, such as in autumn and winter, interception values were as low as 5 per cent. Mean annual interception values were consequently lower than those of the summer months. High percentages of interception did occur with light snowfalls and even in late winter snow depth beneath the trees was less than it was in adjacent open areas. However, since the snow pack in clear-cut areas usually melted earlier than it did beneath the tree canopy, moisture was supplied by melting snow later into the growing season than would have been the case had no tree canopy been present.

The highest percentages of interception occurred in the PseudotsugaTsuga-Hylocomium and Pseudotsuga-Polystichum stands. Tree crowns were large and canopies were dense and two-layered in most of these plots. Interception values in Plots P1 and P2 of the Pseudotsuga-Polystichum association,

$87850-4-5 \frac{1}{2}$ 
howerer, were below average for the association, apparently because stocking in these plots was low. Low stand density could also account for the low interception values of the Thuja-Lysichitum plots. In the Pseudotsuga-Tsuga-Gaultheria and Pseudotsuga-Gaultheria-Peltigera plots, the small size of tree crowns and absence of a dense secondary canopy doubtless contributed to the relatively low interception values recorded. However, the influence of such increased penetration was probably largely negated by more rapid evaporation than would have occurred had less insolation penetrated the tree canopy.

\section{Temperature}

Air and soil-surface temperatures were highest in the open PseudotsugaCraultheria-Peltigera plots (Table V). Soil-surface temperatures reached $124^{\circ} \mathrm{F}$. during summer months, even in undisturbed stands. Still higher values $\left(135^{\circ} \mathrm{F}\right.$.) were recorded within the blackened soil-surface layer of Plot G4 of the Pseudotsuga-Ciaultheria association the summer following a ground fire which destroyed the subordinate regetation. More moderate temperatures were recorded in the denser Pseudotsuga-Tsuga-Hylocomium and Pseudotsuga-Polystichum stands and in the moist Thuja-Lysichitum plots. This trend reflected the moderating influence of stand density and moist soils. The soils of the higher altitude and westerly plots were cooler and warmed up more slowly in spring than their lower altitude and more easterly counterparts. Air and soil-surface temperatures however, were higher than their association averages during summer and autumn

TABLE V

AVERAGE QUARTERLY TEMPERATURES IN PLOTS REPRESENTATIVE OF FOREST ASSOCIATIONS IN THE NANAIMO RIVER VALLEY, BRITISH COLUMBIA, 1951-1953

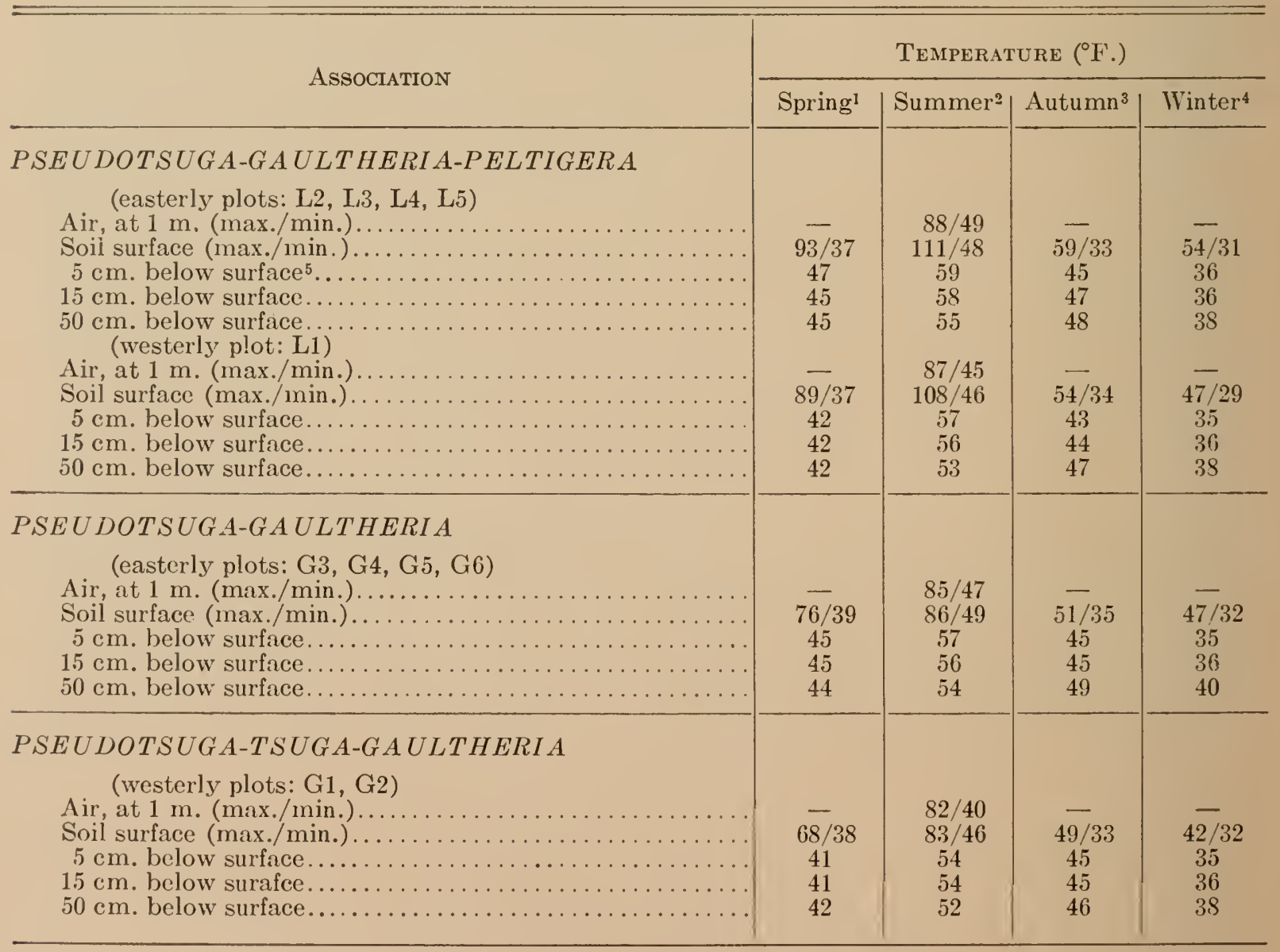




\begin{tabular}{|c|c|c|c|c|}
\hline \multirow{2}{*}{ Association } & \multicolumn{4}{|c|}{ Temperature ( $\left.{ }^{\circ} \mathrm{F}.\right)$} \\
\hline & Spring ${ }^{1}$ & Summer ${ }^{2}$ & Autumn ${ }^{3}$ & Winter ${ }^{4}$ \\
\hline 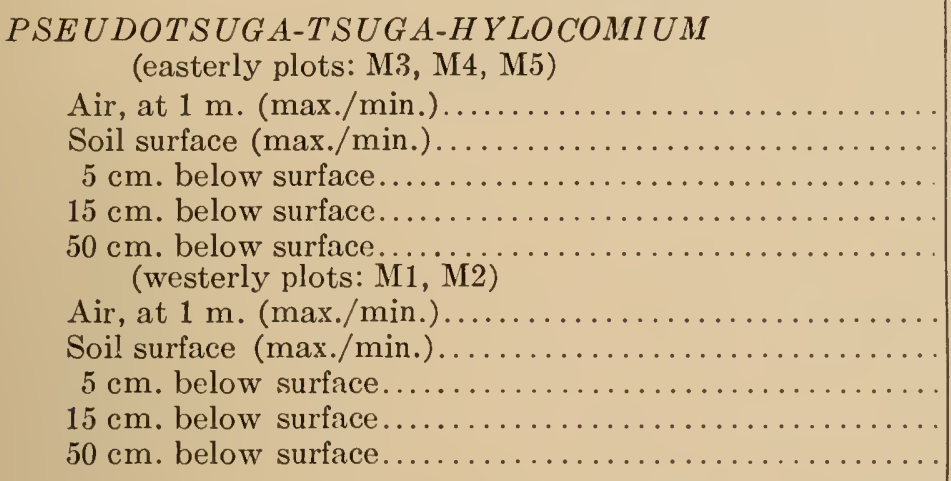 & $\begin{array}{l}- \\
64 / 38 \\
45 \\
43 \\
44 \\
- \\
63 / 38 \\
42 \\
41 \\
41\end{array}$ & $\begin{array}{c}79 / 48 \\
77 / 49 \\
56 \\
55 \\
54 \\
\\
83 / 46 \\
75 / 47 \\
54 \\
53 \\
51\end{array}$ & $\begin{array}{l}- \\
- \\
50 / 35 \\
45 \\
46 \\
47 \\
- \\
49 / 34 \\
43 \\
44 \\
44\end{array}$ & $\begin{array}{l} \\
- \\
46 / 32 \\
36 \\
37 \\
39 \\
- \\
43 / 32 \\
35 \\
35 \\
37\end{array}$ \\
\hline 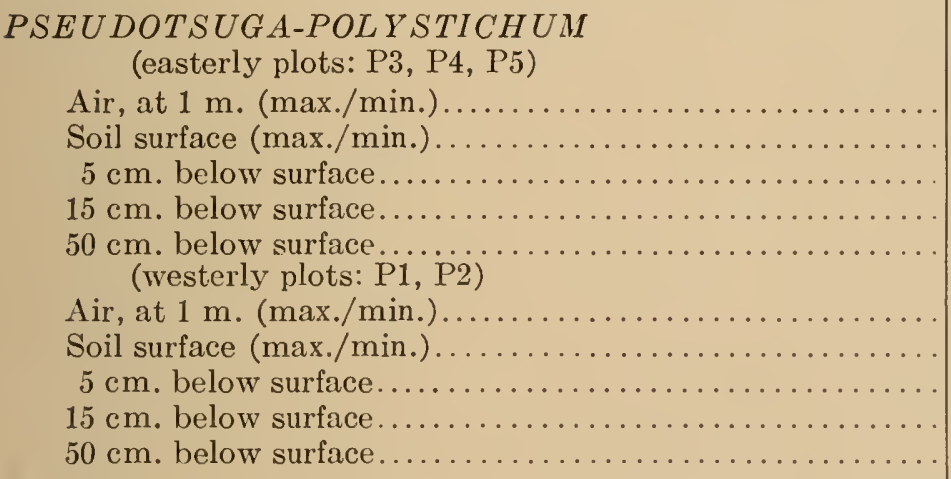 & $\begin{array}{l}- \\
63 / 38 \\
45 \\
43 \\
43 \\
- \\
60 / 39 \\
42 \\
42 \\
41\end{array}$ & $\begin{array}{c}80 / 47 \\
72 / 48 \\
57 \\
55 \\
52 \\
\\
84 / 46 \\
71 / 48 \\
53 \\
53 \\
50\end{array}$ & $\begin{array}{c}- \\
50 / 34 \\
45 \\
46 \\
48 \\
- \\
50 / 34 \\
45 \\
45 \\
48\end{array}$ & $\begin{array}{l}- \\
47 / 32 \\
35 \\
36 \\
39 \\
- \\
42 / 32 \\
35 \\
37 \\
39\end{array}$ \\
\hline 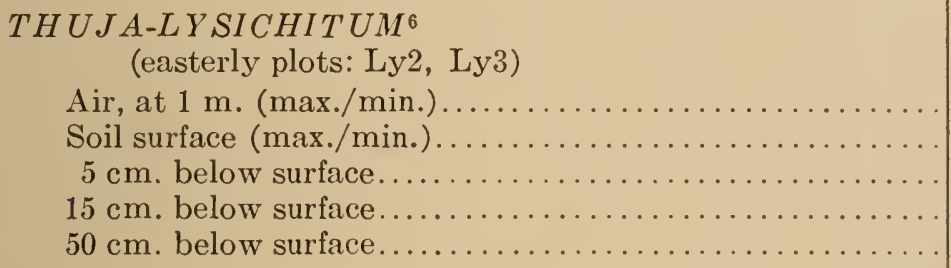 & $\begin{array}{c}- \\
56 / 40 \\
43 \\
44 \\
44\end{array}$ & $\begin{array}{c}80 / 45 \\
65 / 49 \\
53 \\
56 \\
51\end{array}$ & $\begin{array}{c}- \\
48 / 35 \\
43 \\
43 \\
46\end{array}$ & $\begin{array}{l}- \\
44 / 32 \\
44 \\
45 \\
45\end{array}$ \\
\hline
\end{tabular}

${ }^{1}$ Measurements made early in April, May, and June.

${ }_{2}^{2}$ Measurements made early in July, August, and September.

${ }^{3}$ Measurements made early in October, November, and December.

${ }^{4}$ Measurements made early in January, February, and March.

'Subsurface measurements were made at the same time as soil moisture was sampled.

${ }^{6}$ Plot Ly1 was logged before sufficient temperature data had been recorded.

months in the Fourth Lake Pseudotsuga-Tsuga-Hylocomium and PseudotsugaPolystichum Plots, presumably because both stands were adjacent to cut-over areas.

Soil-surface layer's did not remain frozen long enough to impede infiltration appreciably, even though monthly minima at the soil surface fell below $32^{\circ} \mathrm{F}$. Upper soil horizons commonly had monthly minima as low as $34^{\circ} \mathrm{F}$. in winter, but temperatures rarely dropped below $38^{\circ} \mathrm{F}$. at depths of 50 or more centimeter's beneath the soil surface. While such temperatures have been shown to impede water uptake by plants (23), some water must always have been available since subsurface soils were not frozen. 


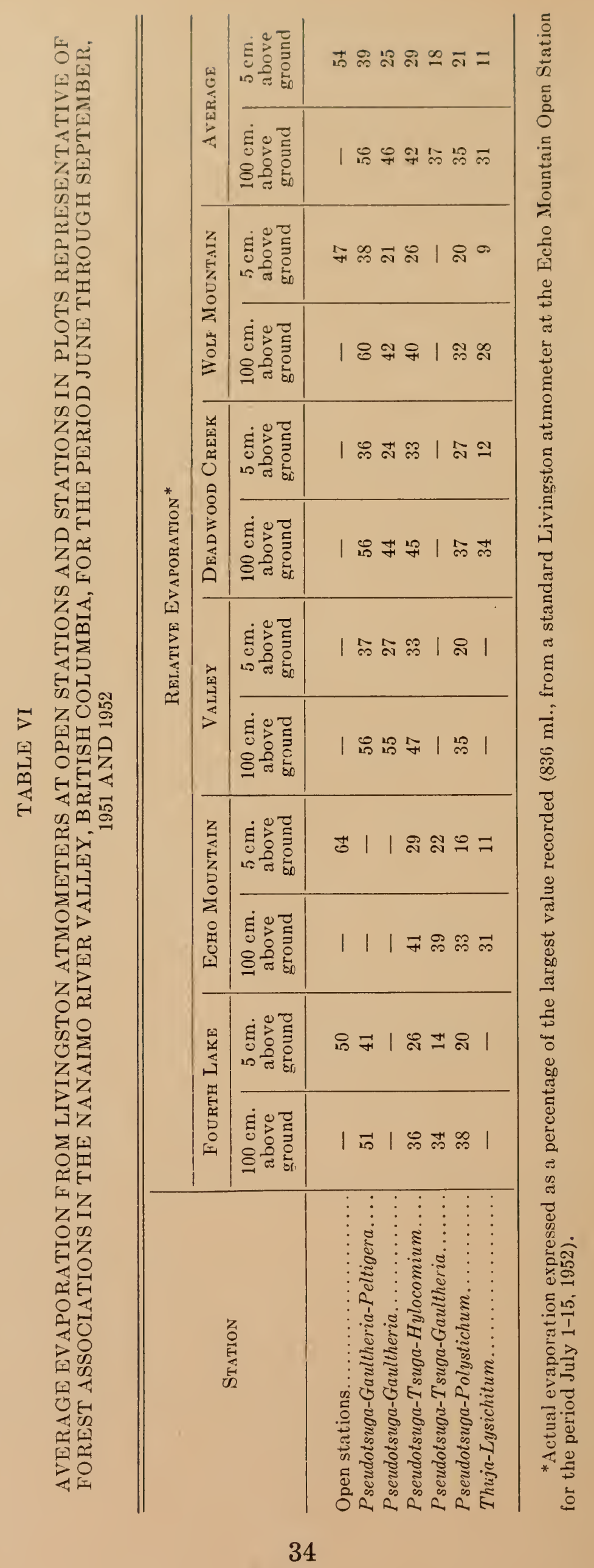




\section{Evaporation}

Evaporation rates were lowest in the moist Thuja-Lysichitum plots and highest at the Open Stations and in the open-canopied Pseudotsuga-GaultheriaPeltigera plots (Table VI). That the high humidity associated with the swampy soils of the Thuja-Lysichitum plots more than counterbalanced the openness of the tree canopy in these plots demonstrated the influence of soil moisture as well as canopy density on evaporation rates. Transpiration loss by the vegetation of subordinate plant layers was presumably similarly influenced, even though evaporation loss from atmometers has been shown to be somewhat disproportionately influenced by wind movement (3).

Plots at higher altitudes commonly had lower evaporation rates than equivalent stands at lower elevations. Evaporation losses at the Fourth Lake Pseudotsuga-Tsuga-Hylocomium Plot, for example, were lower than those of the other plots of this association farther east at lower altitudes. Similarly, despite the openness of their tree canopies, evaporation rates in the Pseudotsuga-TsugaGaultheria plots were among the lowest recorded. The relative frequency of a low cloud ceiling in the westerly portion of the study area in which these stands were located and the reduced insolation characteristic of north slopes, such as the one on which the Fourth Lake Plot was located, doubtless contributed to such conditions. On the other hand, the relatively high Echo Mountain Open Station had the highest rates of any station, apparently because it was located on a shoulder freely exposed to wind movement. Similarly, evaporation rates in the Fourth Lake Pseudotsuga-Polystichum Plot were higher than average for the association. This plot was exposed to abnormal air movement by its proximity to a cut-over area.

The influence of a shrub canopy on evaporation was indicated by the rates from the atmometers $5 \mathrm{~cm}$. above the ground. The Gaultheria shallon canopy apparently exerted an appreciable influence in reducing rates near ground line. At $1 \mathrm{~m}$., rates in the Pseudotsuga-Gaultheria plots were considerably higher than in the more densely canopied Pseudotsuga-Tsuga-Hylocomium stands. At $5 \mathrm{~cm}$. the reverse was true. The absence of subordinate vegetation in Pseudotsuga-Tsuga-Hylocomium stands allowed unrestricted wind movement, and no shrub canopy was present to reduce insolation or raise humidities by transpiration.

The highest evaporation rates occurred in July (Table VII). Rates during the first half of both July and August, 1952, were nearly double those of the

\section{TABLE VII}

AVERAGE MONTHLY EVAPORATION FROM LIVINGSTON ATMOMETERS AT 100 CM. ABOVE THE GROUND IN PLOTS REPRESENTATIVE OF FOREST ASSOCIATIONS IN THE NANAIMO RIVER VALLEY, BRITISH COLUMBIA, JUNE THROUGH SEPTEMBER, 1951-52

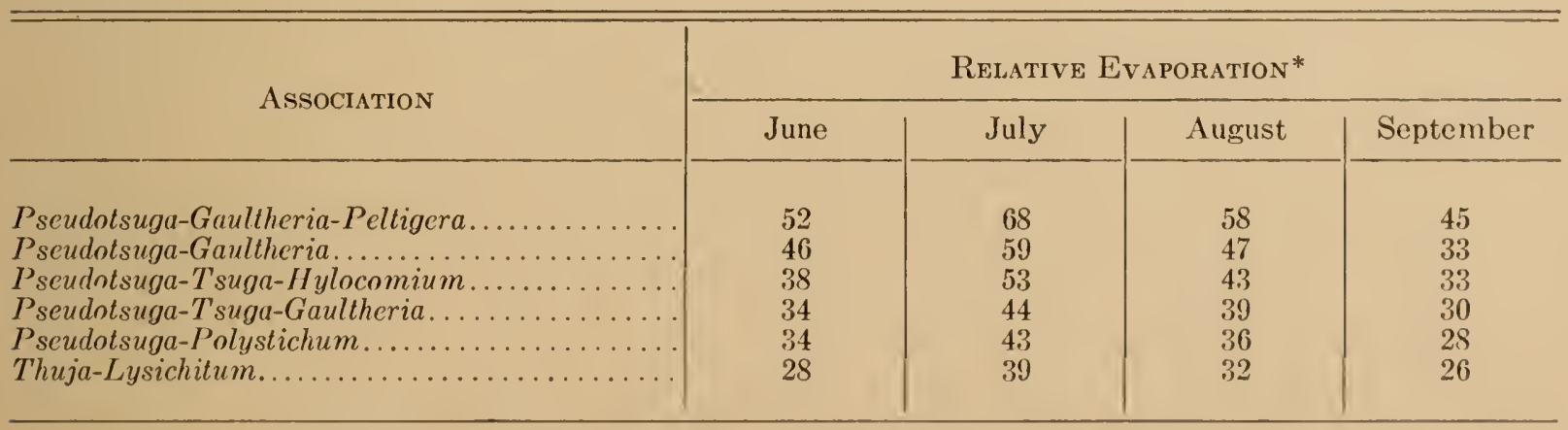

*Actual evaporation expressed as a percentage of the largest value recorded $(836 \mathrm{ml}$., from a standard Livingston atmometer at the Echo Mountain Open Station for the period July 1-15, 1952). 
second half of each month. During these latter periods, rainfall oceurred and cloudy days were more common. Such a difference in rates showed that transpiration usage during the warm, dry summers characteristic of the study area would be considerably higher than in moister, cooler climates outside the lainshadow zone.

\section{Profile Characteristics}

\section{Pseudotsuga-Gaultheria-Peltigera association}

Soil profiles in the Pseudotsuga-Gaultheria-Peltigera plots were usually shallow, being terminated by bedrock or an ortstein layer less than 50 to $70 \mathrm{~cm}$. below the surface (Table VIII, Fig. 13 A and C). Most profiles were gravelly and angular cobbles and small boulders were numerous. Clinker-like and shotty concretions were also common. In many samples, the $2-\mathrm{mm}$. fraction made up less than 50 per cent of the $25-\mathrm{mm}$. fraction and the $2-\mathrm{mm}$. fraction itself was coarse-textured. Such characteristics resulted in profiles with small moisture storage capacities. The infrequency of mottling in any layer showed the soils of these plots to be little affected by seepage moisture.
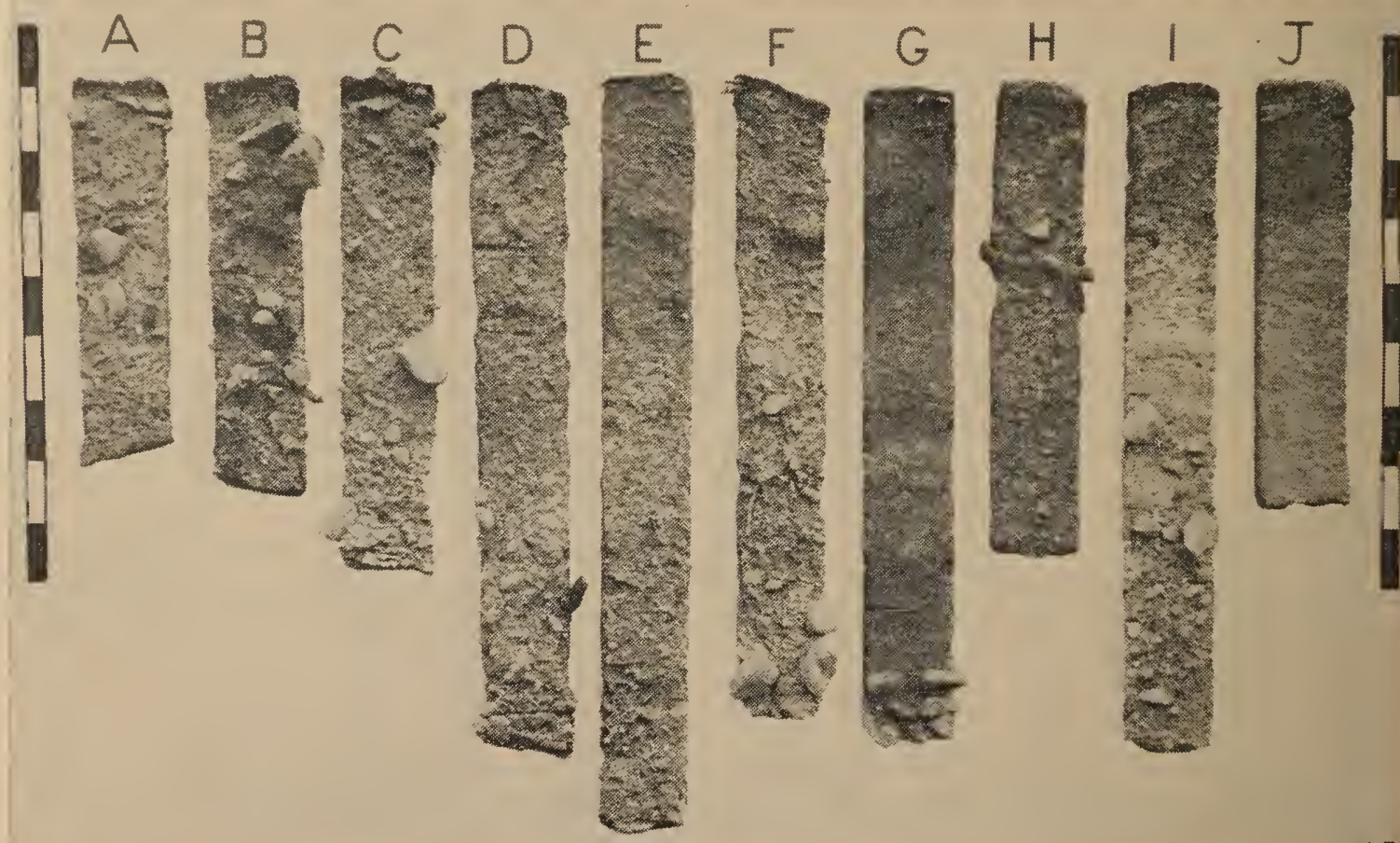

FigURE 13-Typical soil profiles from plots representative of forest associations in the Nanaimo River valley, British Columbia. The scale is marked in decimeters. A. The Pseudotsuga-Gaultheria-Peltigera association (Plot L1 at Fourth Lake). B. The Pseudotsuga-Tsuga-Gaultheria association (Plot G1 at Fourth Lake). C. The Pseudotsuga-Gaultheria-Peltigera association (Plot L3 at Deadwood Creek). D. The Pseudotsuga-Gaultheria association (Plot G6 at Deadwood Creek). E. The Pseudotsuga-Tsuga-Hylocomium association (Plot M5 at Wolf Mountain). F. The PseudotsugaPolystichum association (Plot P1 at Fourth Lake). G. The Pseudotsuga-Polystichum association (Plot P3 in the Valley area). H. The Pseudotsuga-Polystichum association (Plot P2 at Echo Mountain). I. The Pseudotsuga-Polystichum association (Plot P5 at Wolf Mountain). J. The Thuja-Lysichitum association (Plot Ly3 at Wolf Mountain).

The mor-type humus and quite strongly acidic soil $\mathrm{pH}$ values of the Pseudotsuga-Gaultheria-Peltigera plots indicated the relative infertility of their soils. The weak definition of $\mathrm{A}_{2}$ horizons, nevertheless, suggested that leaching was less intense than on some moister sites. 
Profiles in the easterly Plots (L2, L3, L4, and L5) resembled the Shawnigan series described by Day, Farstad, and Laird (10). Most profiles in the Fourth Lake Plot (L1), however, corresponded more closely to the Quinsam series (10). In the moister climate of this westerly portion of the study area, soil colors were brighter and $\mathrm{A}_{2}$ horizons deeper than in the more easterly Pseudotsuga-GaultheriaPeltigera plots. The gravel content of most profiles examined in Plot L1 was also lower than that in the other plots of this association.

\section{Pseudotsuga-Gaultheria association}

The greater depth of material above the ortstein layer in the PseudotsugaGaultheria plots resulted in somewhat larger soil-moisture storage capacities than those of the Pseudotsuga-Gaultheria-Peltigera plots (Table VIII, Fig. 14), even though stones and angular cobbles were equally common and the $2-\mathrm{mm}$. fraction made up scarcely 50 per cent of the $25-\mathrm{mm}$. fraction. Moreover, some mottling was usually present in deeper horizons. Such mottling, together with the stronger color of the dappled, reddish-yellow coatings on cobble and gravel particles showed that the soils of the Pseudotsuga-Gaultheria plots were somewhat more influenced by seepage moisture than those of the PseudotsugaGaultheria-Peltigera plots.

\section{TABLE VIII}

TYPICAL SOIL PROFILES IN PLOTS REPRESENTATIVE OF FOREST ASSOCIATIONS IN THE NANAIMO RIVER VALLEY, BRITISH COLUMBIA

\section{PSEUDOTSUGA-GAULTHERIA-PELTIGERA ASSOCIATION}

Lower Dfadwood Creek (Plot L3)

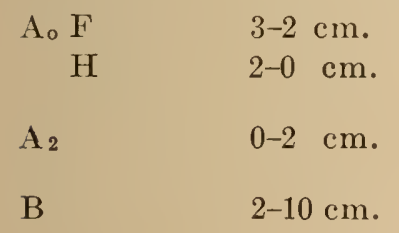

Very dark brown (10YR 2/2)* partially decomposed litter; pH 4.9 .

Black (10YR 2/1) granular to felty mor, up to $4 \mathrm{~cm}$. thick among surface cobbles; pH 5.0 ; roots common.

Gray (5Y 6/1) sandy loam; weak fine subangular blocky structure; very friable; $\mathrm{pH} 4.9$.

Brown (10YR 5/3) gravelly sandy loam, with angular cobbles in places very numerous; weak fine subangular blocky structure; loose; numerous shotty concretions, with yellowish red (5YR 5/8) coatings; light, dappled, strong brown (7.5 YR 5/8) coatings on cobbles and gravel; pH 5.7 ; roots common.

B $\quad 10-20 \mathrm{~cm}$.

Light yellowish brown (10YR 6/4) gravelly sandy loam, with angular cobbles; weak fine subangular blocky structure; very friable; shotty concretions; dappled, strong brown (7.5 YR 5/8) coatings on cobbles and gravel; pH 5.6 ; roots common.

B $20-55 \mathrm{~cm}$.

Pale brown (10YR 6/3) gravelly sandy loam, with scattered angular cobbles; weak fine subangular blocky structure; very friable; shotty concretions; dappled, strong brown (7.5 YR 5/8) coatings on cobbles and gravel; $\mathrm{pH} 5.7$; roots moderately common.

B $\quad 55-70 \mathrm{~cm}$.

Pale olive (5YR 6/3) gravelly sandy loam, with scattered angular cobbles; weak fine subangular blocky structure; very friable, becoming weakly cemented with increasing depth; numerous light olive gray $(5 \mathrm{Y} 6 / 2)$ clinker-like concretions, with yellowish red (5YR 5/8) staining; dappled, yellowish red (5YR 5/8) coatings on cobbles and gravel; $\mathrm{pH} 5.9$; roots sparse, although fine roots common above the ortstein layer.

Ortstein $\quad 70+\mathrm{cm}$.

Fourth Lake (Plot L1)

A。 $F \quad 2-1 \mathrm{~cm}$.

$\mathrm{H} \quad 1-0 \mathrm{~cm}$.

$\mathrm{A}_{2} \quad 0-2 \mathrm{~cm}$.

B 2-20 $\mathrm{cm}$.

Light olive gray (5Y 6/2) gravelly sandy loam; irregular thick platy structure; cemented; reddish yellow (5YR 6/8) staining, particularly in the upper part; $\mathrm{pH} 6.2$; roots absent.

Very dark brown (10YR 2/2) partially decomposed litter.

Black (10YR 2/1) felty mor; pH 4.9; roots common.

Light gray (5Y 7/1) loam, varying from less than $1 \mathrm{~cm}$. to $5 \mathrm{~cm}$. thick among surface cobbles; weak fine subangular blocky structure; pH 4.5; roots common.

Red (2.5YR 4/6) gravelly sandy loam, witl scattered angular cobbles; weak fine subangular blocky structure; friable; shotty concretions, with yellowish red (5YR 5/8) coatings; dappled, yellowish red $(5 \mathrm{YR} 5 / 6)$ coatings on cobbles and gravel; $\mathrm{pH} 5.1$; roots common. 


\section{PSEUDOTSUGA-GAULTHERIA-PELTIGERA ASSOCIATION-Concluded}

Fourtu Lake (Plot L,1)-Concluded

B 20-35 cm. Yellowish red (5YR 5/6) gravelly sandy loam, with scattered angular cobbles; weak fine subangular blocky structure; friable; shotty concretions; dappled, yellowish red (5YR 5/8) coatings on cobbles and gravel; pH 5.2 ; roots moderately common.

B $\quad 35-55 \mathrm{~cm}$.

Reddish yellow (5YR 6/8) gravelly sandy loam, weak fine to medium subangular blocky structure; friable; dappled, reddish yellow (5 Y R 6/8) coatings on cobbles and gravel; scattered, faint blue $(7.5 \mathrm{BG} 7 / 2)$ mottling; pH 5.3; roots sparse, although numerous on rock surface, commonly forming a mat; fine dead roots present.

D) $55+\mathrm{cm}$. Rock, seepage water occasionally present.

\section{PSEUDOTSUGA-GAULTHERIA ASSOCIATION}

Upper Deadwood Creek (Plot G6)

A. $\mathrm{F} \quad 4-2 \mathrm{~cm}$. Very dark brown $(10 \mathrm{YR} 2 / 2)$ partially decomposed litter.

H 2-0 cm. Very dark brown $(10 \mathrm{YR} 2 / 2)$ felty mor; $\mathrm{pH} 5.2$; roots common.

$\mathrm{A}_{2} \quad 0-1 \mathrm{~cm}$.

Dark gray (10YR 4/1) sandy loam; thicker among surface cobbles; weak fine subangular blocky structure; very friable; $\mathrm{pH} 4.8$; roots common.

B $\quad 1-10 \mathrm{~cm}$.

Brown (10YR 5/3) gravelly sandy loam, with scattered angular cobbles; weak fine subangular blocky structure; very friable; numerous shotty concretions, with reddish yellow (7.5 YR 6/6) coatings; dappled, reddish yellow $(7.5 \mathrm{YR} 6 / 6)$ coatings on cobbles and gravel; $\mathrm{pH} 5.7$; roots common.

B $\quad 10-25 \mathrm{~cm}$.

Pale brown (10YR 6/3) gravelly sandy loam, with scattered angular cobbles; weak fine subangular blocky structure; very friable; shotty concretions very numerous; dappled, reddish yellow $(7.5$ Y R 6/6) coatings on cobbles and gravel; pH 5.7; roots common.

B $25-50 \mathrm{~cm}$.

Light yellowish brown $(2.5 \mathrm{Y} 6 / 4)$ gravelly loam, with angular cobbles; weak fine subangular blocky structure; very friable; numerous shotty concretions; light yellowish brown $(2.5 \mathrm{Y} 6 / 4)$ clinker-like coneretions, with reddish yellow (7.5 YR 6/8) staining; dappled, strong brown (7.5YR $5 / 8$ ) coatings on cobbles and gravel; $\mathrm{pH} 5.9$; roots moderately common.

B $\quad 50-80 \mathrm{~cm}$.

Pale olive (5 Y 6/3) gravelly sandy loam, with scattered angular cobbles; weak fine subangular blocky structure; firm to weakly cemented with increasing depth; clinker-like concretions common, becoming numerous towards the ortstein layer; dappled, reddish yellow (5YR 6/8) coatings on cobbles and gravel; reddish yellow (5YR 6/8) staining common above the ortstein; $\mathrm{pH} 6.2$; roots sparse, but fine roots common just above the ortstein.

Ortstein $\quad 80+\mathrm{cm}$.

Olive gray (5Y 5/2) gravelly sandy loam; irregular thick platy structure; cemented; yellowish red (5YR 5/8) staining, prominent in the upper portion; $\mathrm{pH} 6.5$; roots absent.

\section{PSEUDOTSUGA-TSUGA-GAULTHERIA ASSOCIATION}

Есho Mountain (Plot G2)

A。 $\mathrm{F} \quad 5-3 \mathrm{~cm}$.

$\mathrm{H} \quad 3-0 \mathrm{~cm}$.

$\mathrm{A}_{2} \quad 0-4 \mathrm{~cm}$.

B $\quad 4-35 \mathrm{~cm}$.

B

$35-55 \mathrm{~cm}$.
Very dark brown (10YR 2/2) partially decomposed litter.

Very dark brown (10YR 2/2) to black (10YR 2/1) felty mor; pH 4.6; roots forming dense network.

Light gray (10YR 7/1) gravelly sandy loam, in places up to $10 \mathrm{~cm}$. thick among surface cobbles; upper parts often including bleached humus: weak fine subangular blocky structure; very friable; shotty concretions; pH 4.6; roots numerous.

Strong brown (7.5 Y R 5/8) gravelly sandy loam, with scattered angular cobbles; weak fine subangular blocky structure; very friable; shotty concretions common, sometimes with heavy, red (2.5 YR 4/8) coatings; dappled, yellowish red (5YR 5/8) coatings on cobbles and gravel; scattered, faint, yellowish red (5YR 5/8) mottling; pH 5.5; roots common.

Yellowish brown (10YR 5/6) gravelly sandy loam, with scattered angular cobbles; weak fine subangular blocky structure; very friable; shotty concretions common; dappled, faint, reddish yellow (7.5 Y R 6/8) coatings on cobbles and gravel; pH 5.7; roots moderately common. 


\section{PSEUDOTSUGA-TSUGA-GAULTHERIA ASSOCIATION-Concluded}

\section{Echo Mountain (Plot G2)-Concluded}

B $\quad 55-75 \mathrm{~cm}$.

Light yellowish brown (10YR 6/4) gravelly sandy loam, with numerous angular cobbles; weak fine to medium subangular blocky structure; friable; dappled, reddish yellow (7.5YR 6/8) coatings on cobbles; $\mathrm{pH}$ 5.4 ; roots sparse, although more numerous on rock surface, commonly forming a mat.

D $75+\mathrm{cm}$.

Rock; seepage water occasionally present.

(In some profiles the B horizon merged into the $\mathrm{D}$ horizon; in others the root zone was terminated by an ortstein layer).

\begin{tabular}{|c|c|}
\hline A. $F$ & $3-2 \mathrm{~cm}$ \\
\hline $\mathrm{H}$ & $2-0 \mathrm{~cm}$. \\
\hline $\mathbf{A}_{2}$ & $0-2 \mathrm{~cm}$. \\
\hline B & $2-20 \mathrm{~cm}$. \\
\hline B & $20-45 \mathrm{~cm}$ \\
\hline B & $45-75 \mathrm{~cm}$. \\
\hline B & $75-100 \mathrm{cr}$ \\
\hline
\end{tabular}

Ortstein $\quad 100+\mathrm{cm}$.

Fourth LaKe (Plot M1)

A. $\mathrm{F} \quad 7-5 \mathrm{~cm}$.

$\mathrm{H} \quad 5-0 \mathrm{~cm}$.

$\mathrm{A}_{2} \quad 0-6 \mathrm{~cm}$.

B $\quad 6-6.5 \mathrm{~cm}$.

B $\quad 6.5-20 \mathrm{~cm}$.

B

$20-40 \mathrm{~cm}$.

40-65 $\mathrm{cm}$.
Very dark brown (10YR 2/2) partially decomposed litter; $\mathrm{pH} 5.3$.

Very dark brown (10YR 2/2) to black (10YR 2/1) felty mor; $\mathrm{pH} 5.2$; roots moderately common.

Gray (10YR 2/1) sandy loam, up to $10 \mathrm{~cm}$. thick among surface stones; wcak fine subangular blocky structure; very friable; $\mathrm{pH} 5.0$; roots common.

Brown (10YR 5/3) gravelly sandy loam, with numerous angular cobbles and stones; weak fine subangular blocky structure; very friable; shotty concretions common; faint, strong brown (7.5YR 5/8) coatings on cobbles, gravel and shot; $\mathrm{pH} 5.5$; roots common.

Yellowish brown (10YR 5/6) gravelly sandy loam, with angular cobbles weak fine subangular blocky structure; very friable; shotty concretions occasional pale olive (5YR 6/3) clinker-like concretions, with yellowish red (5YR 5/6) staining; dappled, strong brown (7.5YR 5/8) coatings on cobbles and gravel; $\mathrm{pH} 5.3$; roots moderately common.

Yellowish brown (10YR 5/6) gravelly loamy sand, with angular cobbles; weak fine subangular blocky structure; friable; shotty concretions : clinker-like concretions more common; strong brown (7.5 Y R 5/8) coatings on cobbles and gravel; $\mathrm{pH} 5.8$; roots sparse.

Yellowish brown (10YR 5/8) gravelly loamy sand, with scattered angular cobbles; weak fine subangular blocky structure; becoming weakly cemented; shotty concretions; clinker-like concretions nearly continuous strong brown (7.5YR 5/8) coatings and mottling; faint bluish (7.5BG 7/2) mottling; pH 5.4; roots sparse.

Olive gray (5Y 5/2) gravelly sandy loam; irregular thick platy structure; cemented; yellowish red (5YR 4/8) staining; no roots.

Very dark brown (10YR 2/2) partially decomposed litter; pH 4.7.

Very dark brown (10YR 2/2) to black (10YR 2/1) felty mor; pH 4.2; roots numerous. In places the surface horizon consistcd of up to $15 \mathrm{~cm}$. of decayed wood.

Light brownish gray $(2.5 \mathrm{Y} 6 / 2)$ sandy loam; up to $15 \mathrm{~cm}$. thick under decayed wood; weak fine subangular blocky structure; very friable; pH 4.3 ; roots common.

Dark reddish brown (5YR 3/4) loam; discontinuous.

Reddish brown (5YR 5/4) gravelly sandy loam, with angular cobbles and stoncs; weak fine subangular blocky structure; very friable; shotty concretions with yellowish red (5YR 5/8) coatings; dappled, yellowish red (5YR 5/8) coatings on cobbles and gravel; $\mathrm{pH} 5.3$; roots common.

Reddish brown (5YR 5/4) gravelly sandy loam, with angular cobbles; weak fine subangular blocky structure; friable; shotty concretions common; occasional light ycllowish brown (2.5 Y 6/4) clinker-like concretions, with heavy, yellowish red (5YR 4/8) staining; heavy, dappled reddish yellow (5 YR 6/8) staining on cobbles and gravel; pII 5.3; roots common.

Brown (7.5 Y R 5/4) gravelly sandy loam, with angular cobbles and stones; weak fine subangular blocky structure; friable; shotty concretions: clinker-like concretions more common; heavy dappled, yellowish red (5YR 5/8) staining on cobbles and gravel; faint bluish $(7.5 \mathrm{BG} 7 / 2)$ mottling; pH 5.3 ; roots moderatcly common. 


\section{PSELDOTSUGA-TSLGA-HYLOCOMUM ASSOCIATION-Concluded}

Foźnт Lake (Plot M1)-Concluded

B 65-90 $\mathrm{cm}$.

Ortstein $\quad 90+\mathrm{cm}$.

Lower Deadwoon (Plot M4)

$\begin{array}{ccc}A_{0} \mathrm{~F} & 3-2 & \mathrm{~cm} . \\ \text { HI } & 2-0 & \mathrm{~cm} . \\ \mathrm{A}_{2} & 0-1 & \mathrm{~cm} .\end{array}$

B $1-10 \mathrm{~cm}$.

B $\quad 10-20 \mathrm{em}$.

B $20-40 \mathrm{em}$.

B $\quad 40-70 \mathrm{em}$.

B $\quad 70-100 \mathrm{~cm}$.

Echo Mountain (Plot M2)

$\begin{array}{lll}\text { A. } F & 2-1 & \mathrm{~cm} . \\ \mathrm{H} & 1-0 & \mathrm{~cm} . \\ \mathrm{A}_{2} & 0-1 & \mathrm{~cm} . \\ \mathrm{B} & & \end{array}$

B

20-40 cm.

B $\quad 40-75 \mathrm{~cm}$.

B (G) $\quad 75-100 \mathrm{em}$.

Wolf Mountain (Plot M5)

$\begin{array}{ccc}\text { A. } \mathrm{F} & 2-1 & \mathrm{~cm} . \\ \mathrm{H} & 1-0 & \mathrm{~cm} . \\ \mathrm{A}_{2} & 0-1 & \mathrm{~cm} .\end{array}$

B $1-10 \mathrm{~cm}$.
Yellowish brown (10YR 5/6) gravelly sandy loam, witl angular cobbles and stones; weak fine to medium subangular blocky structure; friable; shotty concretions; clinker-like concretions numerous; heavy, dappled vellowish red (5Y R 5/8) coatings on eobbles and gravel; scattered bluish (7.5BG 7/2) mottling; p H 5.7; roots sparse, although fine roots common above ortstein layer.

Olive gray (5 Y 5/2) gravelly sandy loam; irregular thick platy structure; cemented; yellowish red (5 Y R 4/8) staining; no roots.

Very dark brown (10YR 2/2) partially decomposed litter; pH 5.3.

Black (10YR 2/1) felty inor; pH 5.5; roots eommon.

Gray (10YR 5/1) sandy loam, often thin but well defined; weak fine subangular blocky structure; very friable; pH 5.6.

Brown (7.5YR 5/4) gravelly loany sand, with angular cobbles; weak subangular blocky structure; very friable; numerous shotty concretions; faint, strong brown (7.5 YR 5/8) eoatings on eobbles, gravel, and shot; pH 6.0 ; roots common.

Yellowish brown (10 YR 5/4) gravelly loamy sand, with seattered cobbles; weak fine subangular blocky structure; very friable; numerous shotty concretions; faint, strong brown ( 7.5 YR 5/8) coatings on cobbles, gravel, and shot; $\mathrm{pH} 6.0$; roots common.

Yellowish brown (10YR 5/6) gravelly sandy loam, with scattered cobbles and stones; weak fine subangular blocky structure; very friable; shotty concretions common; light yellowish brown (2.5Y 6/4) clinker-like concretions, with faint, strong brown (7.5 YR 5/8) staining; faint, strong brown (7.5YR 5/8) coatings on eobbles, gravel, and shot; very slight bluish (7.5BG 7/2) mottling; $\mathrm{pH} 6.0$; roots moderately com mon.

Yellowish brown (10YR 5/8) gravelly loamy sand, with scattered cobbles; weak fine subangular blocky structure; very friable; shotty and clinkerlike concretions common; faint, strong brown (7.5 YR 5/8) coatings on cobbles, gravel, and shot; $\mathrm{pH} 6.7$; roots sparse.

Light yellowish brown (2.5Y 6/4) gravelly loamy sand, with eobbles; very weak granular structure; loose; cobbles and gravel largely unstained pH 6.2 ; roots sparse.

Very dark brown (10YR 2/2) partially decomposed litter; $\mathrm{pH}$ 5.2.

Very dark brown (10YR $2 / 2)$ felty mor; roots moderately common.

Dark gray (10YR 4/1) sandy loam, in places poorly defined; weak fine subangular blocky structure; very friable; $\mathrm{pH} 5.7$.

Yellowish red (5YR 5/8) gravelly sandy loam, with angular cobbles; weak fine subangular blocky structure; friable; shotty concretions common; dappled, strong brown $(7.5 \mathrm{YR} 5 / 8)$ coatings on cobbles, gravel, and shot; $\mathrm{pH} 5.6$; roots common.

Yellowish red (5YR 5/8) gravelly sandy loam, with scattered angular cobbles; weak fine subangular blocky structure; friable; shotty concretions; strong brown (7.5YR 5/8) coatings on cobbles, gravel, and shot; pH 5.9 ; roots eommon.

Strong brown (7.5 YR 5/6) graveliy sandy loam, with seattered angular cobbles; weak fine subangular blocky structure; friable; shotty concretions; strong brown ( $7.5 \mathrm{Y}$ R 5/8) coatings on cobbles, gravel, and shot; pH 5.9 ; roots sparse.

Yellowish brown (10YR 5/6) gravelly sandy elay loam, with stones and angular cobbles; medium subangular blocky structure; firm; strong brown (7.5 Y R 5/8) eoatings on cobbles and gravel; faint bluish $(7.5 \mathrm{BG}$ $7 / 2$ ) mottling; pH 5.7; roots sparse.

Very dark brown (10YR 2/2) partially decomposed litter; $\mathrm{pH} 5.5$.

Black (10YR 2/1) duff mull; pH 5.5; roots moderately common.

Very dark brown (10YR 2/2) loam; weak crumb structure; friable; $\mathrm{pH} 5.7$; roots moderately common.

Red (2.5YR 4/6) loam; weak medium subangular blocky strueture; friable; shotty coneretions, with yellowish red (5YR 5/6) eoatings; diffuse, yellowish red coatings on gravel; pHI 6.4; roots common. 


\section{PSEUDOTSUGA-TSUGA-HYLOCOMIUM ASSOCIATION-Concluded}

\section{Wolf Mountain (Plot M5)-Concluded}

B

$10-20 \mathrm{~cm}$.

$\mathrm{B}$

$20-45 \mathrm{~cm}$.

$\mathrm{B}$

$45-70 \mathrm{~cm}$.

B

$70-100 \mathrm{~cm}$.

D

$100-130 \mathrm{~cm}$.
Yellowish red (5YR 4/6) sandy loam; weak fine subangular blocky structure; friable; numerous shotty concretions; yellowish red $(5 \mathrm{YR} 5 / 6)$ coatings on gravel and shot; $\mathrm{pH} 6.5$; roots common.

Yellowish red (5YR 5/6) sandy loam; weak fine subangular blocky structure; friable; numerous shotty concretions; yellowish red (5YR 5/6) coatings on gravel and shot; $\mathrm{pH} 6.4$; roots moderately common.

Yellowish brown (10YR 5/8) sandy loam; weak fine subangular blocky structure; friable; shotty concretions; scattered, red (2.5Y R 5/8)coatings on gravel; pH 6.1 ; roots moderately common.

Light olive brown (2.5Y 5/4) sandy loam; weak fine subangular blocky structure; friable to firm; red $(2.5 \mathrm{YR} 5 / 7)$ coatings on gravel and scattered red staining above compact lorizon below; faint, bluish $(7.5 \mathrm{BG}$ $7 / 2$ ) cast and weak, red mottling; $\mathrm{pH} 6.3$; roots sparse, although some fine roots present just above the compact horizon.

Pale olive (5Y 6/4) sandy loam; weak medium subangular blocky structure; firm to weakly cemented; compact; diffuse, red (2.5 YR 5/8) coatings on gravel; red staining and mottling; pH 6.1; roots absent.

\section{PSEUDOTSUGA-POLYSTICHUM ASSOCIATION}

\section{Upper Deadwood Creek (Plot P4)}

\section{A。 $\mathrm{F} \quad 3-2 \mathrm{~cm}$. \\ Very dark brown (10YR 2/2) partially decomposed litter; pH 5.5.}

$\mathrm{H} \quad 2-0 \mathrm{~cm}$.

$\mathrm{A}_{1} \quad 0-1 \mathrm{~cm}$.

B $1-10 \mathrm{~cm}$.

Black (10YR 2/1) duff mull to granular mor; $\mathrm{pH} 5.2$; roots common.

Black (10YR 2/1) sandy loam, somewhat discontinuous; crumb structure; friable; roots numerous.

Dark reddish brown (5YR 3/3) gravelly sandy loam, with occasional rounded cobbles; weak fine subangular blocky structure; friable; scattered shotty concretions; scattered, faint, strong brown (7.5 YR 5/8) coatings on cobbles; $\mathrm{pH} 6.3$; roots common.

B

$10-20 \mathrm{~cm}$.

B

$20-40 \mathrm{~cm}$.

B

$40-50 \mathrm{~cm}$.

B

50-75 cm.

B

$75-100 \mathrm{~cm}$.

B

$100-130 \mathrm{~cm}$.

$\begin{array}{ll}\text { Fourth LaKe } & \text { (Plot P1) } \\ \text { A。 F } & 4-3 \mathrm{~cm} . \\ \text { H } & 3-0 \mathrm{~cm} . \\ \mathrm{A}_{2} & 0-1 \mathrm{~cm} .\end{array}$

B

1-4 cin.

B

4-20 cm.

Yellowish brown (10YR 5/6) sandy loam, with occasional rounded gravel and cobbles; weak fine subangular blocky structure; friable; scattered shotty concretions; scattered, faint, strong brown (7.5 YR 5/8) coatings on cobbles; $\mathrm{pH} 6.3$; roots common.

Yellowish brown (10YR 5/8) sandy loam, with occasional rounded gravel and cobbles; very weak fine subangular blocky structure; very friable; scattered shotty concretions; scattered, faint, strong brown $(7.5$ YR 5/8) coatings on cobbles and gravel; $\mathrm{pH} 6.1$; roots common.

Gray and brown sand, rounded gravel and cobbles; single grain structure; loose; scattered, faint, strong brown ( $7.5 \mathrm{YR} 5 / 8)$ coatings on cobbles and gravel; roots sparse.

Strong brown (7.5 YR 5/6) gravelly sandy loam, with scattered rounded coarse gravel and cobbles; weak fine subangular blocky structure; very friable; scattered, faint, strong brown ( $7.5 \mathrm{YR} 5 / 8)$ coatings on cobbles and gravel; pH 6.4 ; roots moderately common.

Yellowish brown (10YR 5/7) gravelly loamy sand, with scattered rounded coarse gravel and cobbles; very weak finc subangular blocky structure; scattcred, faint, strong brown (7.5YR 5/8) coatings on cobbles and gravel; pH 6.3 ; roots sparse.

Light yellowish brown (2.5Y 6/4) gravelly loamy sand, with scattcred rounded coarse gravel and cobbles; very weak fine subangular blocky structure; very friable; scattercd, faint, strong brown (7.5 YR 5/8) coatings on cobbles and gravel; $\mathrm{pH} 6.2$; roots very sparse.

Very dark brown (10YR 2/2) partially decomposed litter; pH 5.0.

Very dark brown (10YR 2/2) felty mor; pH 4.5; roots common.

Reddish brown (5YR 5/3) sandy loam; weak finc subangular blocky structure; friable; shotty concretions; roots common; this horizon merges into the next.

Yellowish red (5YR 5/6) gravelly sandy loam, often discontinuous; weak fine subangular blocky structure; friable; numerous shotty concretions, with heavy, ycllowish red (5 Y R 5/8) coatings; roots commion.

Yellowish red (5YR 4/6) gravelly sandy loam, with scattered angular cobbles; weak finc to medium subangular blocky structure: friable; numerous shotty concretions; scattered, reddish yellow (5YR 6/8) coatings on gravel and shot; pH 5.3; roots common. 


\section{PSEUDOTSUGA-POLYSTICHUM ASSOCIATION-Continued}

Foumti Lake (Plot P1)-Concluded

B $20-45 \mathrm{~cm}$.

B

B

$100-120 \mathrm{~cm}$

\section{Echo Mountain (Plot P2) \\ A。 $\mathrm{F} \quad 8-6 \mathrm{~cm}$. \\ $\mathrm{H} \quad 6-0 \mathrm{~cm}$.}

$\begin{array}{lll}\text { A } & 0-1 \quad \mathrm{~cm} . \\ \mathrm{B} & 1-3 \mathrm{~cm} .\end{array}$

B $3-10 \mathrm{~cm}$.

B $\quad 10-20 \mathrm{~cm}$.

B $20-50 \mathrm{~cm}$.

B $\quad 50-75 \mathrm{~cm}$.

Ortstein $\quad 75+\mathrm{cm}$

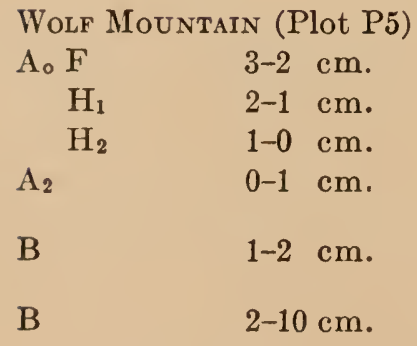

$10-25 \mathrm{~cm}$.

$25-40 \mathrm{~cm}$

Strong brown (7.5 Y R 5/6) gravelly loam, with seattered angular cobbles; weak medium subangular blocky structure; friable; shotty concretions; seat tered, reddish yellow (5Y R 6/8) coatings on gravel and shot; pH 5.6; roots moderately common.

Yellowish brown (10YR 5/6) gravelly loam, with seattered angular cobbles; weak medium subangular blocky strueture; friable; diffuse, reddish yellow (5YR 6/8) coatings on cobbles and gravel; pH 5.8; roots common.

Yellowish brown (10YR 5/8) gravelly loam, with scattered stones and angular cobbles; weak medium to coarse subangular blocky structure; firm; faint, dappled, ycllowish red (5YR 5/8) coatings on gravel and cobbles; $\mathrm{pH} 5.9$; roots sparse.

Light yellowish brown (2.5Y 6/4) gravelly loam, with stones and angular cobbles; weak coarse subangular blocky structure; slightly plastic when moist; very firm when dry; dappled, yellowish red (5YR 5/8) coatings on cobbles and gravel; $\mathrm{pH} 5.6$; roots sparse.

Very dark brown (10YR 2/2) partially decomposed litter; $\mathrm{pH} 5.4$.

Very dark brown (10YR 2/2) duff mull to felty mor; often includes dark reddish brown $(2.5 \mathrm{YR} 3 / 4)$ woody peat from decayed wood; where peat absent humus about $2 \mathrm{~cm}$. deep; $\mathrm{pH} 5.4$; roots common.

Dark reddish gray (5YR 4/2) loam; under peaty decayed wood, this horizon may be up to $10 \mathrm{~cm}$. thick; weak fine subangular blocky structure; friable; roots common.

Dark red (2.5YR 3/6) sandy loam; weak fine subangular blocky structure; friable; shotty concretions with heavy, red $(2.5 \mathrm{YR} 5 / 8)$ coatings; dappled, yellowish red (5YR 5/8) coatings on gravel; pH 5.6; roots numerous.

Red (2.5YR 4/8) sandy loam; weak fine to medium subangular blocky structure; numerous shotty concretions; yellowish red $(5 \mathrm{YR} 5 / 8)$ coatings on gravel and shot; $\mathrm{pH} 5.9$; roots common.

Yellowish red (5YR 4/8) sandy loam; weak fine to medium subangular blocky structurc; friable; numerous shotty concretions; yellowish red (5YR 5/8) coatings on gravel and shot; $\mathrm{pH} 6.1$; roots common.

Yellowish red (5YR 4/8) sandy loam; weak fine to medium subangular blocky structure; friable; shotty concretions; yellowish red (5YR 5/8) coatings on gravel and shot; $\mathrm{pH} 6.0$; roots moderately common.

Red (2.5YR 4/6) sandy loam; weak fine to medium subangular blocky structure; friable; shotty concretions; diffuse, yellowish red (5YR 5/8) coatings on gravel; roots sparse. This horizon is commonly below the water table; $\mathrm{pH} 6.1$.

Olive gray (5Y 5/2) sandy loam; irregular thick platy structure; cemented; prominently mottled with yellowish red (5YR $5 / 8)$ on exposure toair; roots absent.

Very dark brown (10YR 2/2) partially decomposed litter.

Very dark brown (10YR 2/2) felty mor; roots common.

Black (10YR 2/1) granular duff mull; roots common.

Dark gray brown (10YR 4/2) loam, often discontinuous and poorly defined; weak fine subangular blocky structure; friable; roots common.

Dark reddish brown (5YR 3/2) loam, often discontinuous; weak fine subangular blocky structure; friable; roots common.

Dark red (2.5YR 3/6) sandy loam; weak fine to medium subangular blocky structure; friable; numerous shotty concretions with yellowish red (5YR 5/8) coatings; $\mathrm{pH} 6.0$; roots numerous.

Red (2.5YR 4/8) sandy loam; weak fine to medium subangular blocky structure; friable; numerous shotty concretions, with yellowish red coatings; $\mathrm{pH} 6.2$; roots common.

Yellowish red (5YR 5/6) sandy loam; weak fine subangular blocky structure; friable; shotty concretions common; $\mathrm{pH} 6.1$; roots common.

Yellowish brown (10YR 5/7) gravelly sandy loam; weak fine subangular blocky structure; friable; occasional shotty concretions with bright, yellowish red ( $5 \mathrm{Y} \mathrm{R} 5 / 8$ ) coatings; scattered, faint, yellowish red (5YR $5 / 8$ ) coatings on gravel; scattered, distinct, yellowish red (5YR 5/8) mottling; $\mathrm{pH} 6.1$; roots sparse. 


\section{PSEUDOTSUGA-POL YSTICHUM ASSOCIATION-Concluded}

Wolf Mountain (Plot P5)-Concluded

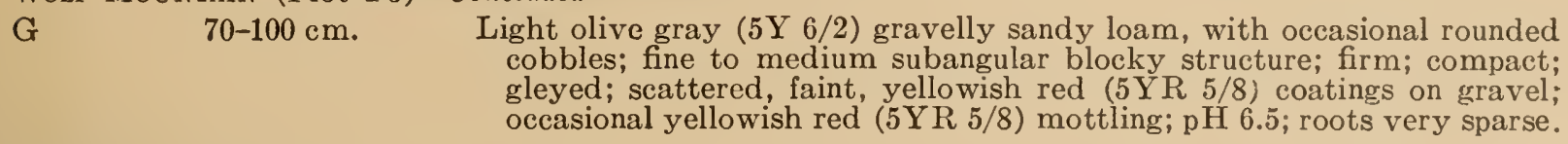

\section{VAlley (Plot P3)}

\begin{tabular}{|c|c|}
\hline$A_{0} F$ & $1.5-0.5 \mathrm{~cm}$. \\
\hline $\mathrm{H}$ & $0.5-0 \mathrm{~cm}$ \\
\hline$A_{1}$ & $0-2 \mathrm{~cm}$. \\
\hline B & $2-3 \mathrm{~cm}$. \\
\hline B & $3-8 \mathrm{~cm}$. \\
\hline B & $8-20 \mathrm{~cm}$. \\
\hline B & $20-50 \mathrm{~cm}$. \\
\hline B & 50-70 cm. \\
\hline B & $70-100 \mathrm{~cm}$. \\
\hline
\end{tabular}

Very dark brown (10YR 2/2) partially decomposed litter.

Very dark brown (10YR 2/2) duff mull; $\mathrm{pH} 5.1$; roots common.

Black (10YR 2/1) loam; weak fine to medium crumb structure; friable; $\mathrm{pH} 5.2$; roots common.

Very dark brown (10YR 2/2) loam; fine subangular blocky structure; friable; occasional shotty concretions; roots common.

Dark brown (10YR 3/3) loam; weak fine subangular blocky structure; friable; occasional shotty concretions; $\mathrm{pH} 5.6$; roots common.

Dark yellowish brown (10YR 4/4) sandy loam; very weak fine subangular blocky structure; very friable; $\mathrm{pH} 5.4$; roots common.

Yellowish brown (10YR 5/6) sandy loam; very weak fine subangular blocky structure; very friable, with bands of loose sand; $\mathrm{pH} 6.2$; roots moderately common.

Dark yellowish brown (10YR 4/4) loam; weak fine subangular blocky structure; very friable; $\mathrm{pH} 6.0$; roots common. This horizon may be the upper part of a buried profile.

Light gray and brown, raw, river gravel and sand; single grain structure; loose; occasional, light, dappled, strong brown (7.5 YR 5/8) coatings on gravel; $\mathrm{pH} 6.2$; roots very sparse.

\section{THUJA-LYSICHITUM ASSOCIATION}

Wolf Mountain (Plot Ly3)

A: SWAMP

$$
\begin{aligned}
& 0-1 \mathrm{~cm} \text {. } \\
& 1-20 \mathrm{~cm} \text {. }
\end{aligned}
$$

$20-23 \mathrm{~cm}$.

$23-35 \mathrm{~cm}$.

\section{B: BANKS AND HUMMOCKS}

$$
\begin{aligned}
& 0-1 \mathrm{~cm} . \\
& 1-2 \mathrm{~cm} \text {. } \\
& 2-20 \mathrm{~cm} \text {. }
\end{aligned}
$$

$20-40 \mathrm{~cm}$

$40+\mathrm{cm}$.
Moss and debris (needles, twigs, wood fragments).

Black muck; water level varying from 0 to $10 \mathrm{~cm}$. below the moss layer; pH 5.6.

Olive gray (5Y 4/2) mucky sandy loam; firm; compact; roots sparse (mostly Lysichitum).

Olive gray (5Y 5/2) gleyed sandy loam; plastic; slightly sticky when wet; firm; compact; hard when dry; brown and yellowish red mottling (faint before exposure to air); pH 6.8; roots absent.

Partially decomposed litter.

Very dark brown (10YR 2/2) felty mor; $\mathrm{pH} 4.7$.

Dark red (2.5YR $3 / 6)$ fibrous peat; contains twig and wood fragments; roots forming a dense network.

Very dark brown (10YR 2/2) to black muck; pH 4.2: roots sparse.

Olive gray (5Y 5/2) gleyed gravelly sandy loam; upper portion heavily infiltrated with muck; slightly plastic; slightly sticky; firm; compact; hard when dry; water commonly running over the surface; $\mathrm{pH} 6.8$; roots absent.

*Color descriptions are according to the Munsell Soil Color Chart (Munsell Color Company, Inc., Baltimore, Maryland, U.S.A.) and refer to soils in the moist state.

The greater depth of litter layers in the Pseudotsuga-Gaultheria plots seemed partly attributable to the forest floor being cooler and moister than in the Pscudotsuga-Gaultheria-Peltigera plots. Although somewhat more productive than Pseudotsuga-Gaultheria-Peltigera sites, the mor-type humus and acidic soil $\mathrm{pH}$ values of the Pseudotsuga-Gaultheria plots suggested that their soils were not particularly fertile. The deeper $\mathrm{A}_{2}$ horizons of the Pseudotsuga-Gaultheria plots indicated that leaching was more intense than in the Pseudotsuga-GaultheriaPeltigera plots. 
Soil profiles in the Wolf Mountain and Deadwood Creek Plots (G4, G5, and G6) Were comparable with the Shawnigan series (10). On the other hand, most profiles in the Valley Plot (G3) resembled the Sproat series (10), because their color's were brighter and their $\mathrm{A}_{2}$ horizons more distinct than those of the more eisterly plots.

\section{Pscudotsuga-T'suga-Gaultheria association}

Soil depth in most of the profiles examined in the Pseudotsuga-T'sugaGaultheria plots was limited by bedrock less than $80 \mathrm{~cm}$. below the surface. Soil-moisture storage capacity was further limited by the frequency of small boulders, angular cobbles, and shotty and clinker-like concretions (Table VIII, Fig. 13 B). The occurrence of mottling nerertheless showed that the soils of these plots were affected by seepage moisture.

'The deep litter layers characteristic of the Pseudotsuga-T'suga-Gaultheria plots were indicative of the cool and moist condition of the forest floor during much of the year. The mor character of these litter layers, the deep $\mathrm{A}_{2}$ horizons, and strongly acidic soil pH values of these plots indicated the intensity of leaching and relative infertility of their soils.

Soil profiles in the Pseudotsuga-Tsuga-Gaultheria plots corresponded to the Quisam series of Day, Farstad, and Laird (10).

\section{Pseudotsuga-Tsuga-Hylocomium association}

Soil profiles in the typicum plots were characterized by their coarse texture and the clear differentiation of $\mathrm{A}_{2}$ horizons. In the Fourth Lake and Valley Plots (M1 and M3) up to 40 per cent of soil volume was occupied by small boulders, angular cobbles, and clinker-like concretions over $25 \mathrm{~mm}$. in diameter. The last were particularly common toward the base of profiles. Mottling, indicative of the periodic occurrence of seepage moisture, was present in the lower profile of all soil pits examined on Plots M1 and M3 (Table VIII). However, seepage moisture in such coarse-textured profiles was probably less influential than in finer-textured soil because surface layers remained essentially unaffected and rapid internal drainage limited the duration of supplements to soil-moisture reserves. The deep $A_{2}$ horizon and bright color of profiles in the Fourth Lake Plot (M1) were characteristics that these profiles had in common with the Stamp series of Day, Farstad, and Laird (10). Such characteristics are typical of profile development in a wet climate. Soil profiles in Plot M3 were similar to the Sproat series (10), another soil developed under quite wet climatic conditions. The soils of Plot M4, being derived from coarse outwash material, resembled the Qualicum series (10). The shallow $\mathrm{A}_{2}$ horizon of such profiles is typical of soil development under relatively dry climatic conditions.

In contrast to the typicum plots, soil profiles in the nudum plots were characterized by relatively fine soil textures and little or no development of $\mathrm{A}_{2}$ horizons. In the Wolf Mountain Plot (M5), the upper 90 to $100 \mathrm{~cm}$. of the profile consisted of fine outwash material, nearly 90 per cent of which was included within the $5-\mathrm{mm}$. soil fraction (Fig. $13 \mathrm{E}$ ). While the soils of the Echo Mountain Plot (M2) contained angular cobbles and small boulders, clay contents were higher than those of soils in the typicum plots. Both plots consequently had quite high soil-moisture storage capacities. Downward percolation of water was restricted by a compact layer in the lower profile of both plots. The mottling present above this layer indicated the occurrence of high moisture contents during part of the year. Wilde (46) designates such profiles as gamma-gley soils.

The occurrence of an $A_{1}$ horizon with a crumb structure on Plot M5 was indicative of a relatively fertile soil. Even in Plot M2, litter layers were shallow 
and the $\mathrm{A}_{2}$ horizon weakly defined. Both characteristics suggest that seepage moisture was relatively effective in amcliorating the leaching influence of the wet climate in this section of the study area (20).

Most of the profiles examined on Plot M2 were comparable with the Alberni series, although those developed from relatively coarse material resembled the Stamp series more closely (10). Profiles in Plot M5 were similar to the Royston series $(10)$.

\section{Pseudotsuga-Polystichum association}

While the soil parent-material and topographic position of the PseudotsugaPolystichum plots were diverse, profile development was such that the soils of all plots supported a similar vegetation type. Salient profile characteristics were the duff-mull character of litter layers, the weak differentiation of $\mathrm{A}_{2}$ horizons, and the moderate aciaity of $\mathrm{pH}$ values, all features denoting relatively fertile soils. Furthermore, the fairly high proportion of fine material included in all profiles resulted in quite high soil-moisture storage capacities. The mottling evident in most profiles indicated that moisture reserves were also supplemented by seepage moisture.

Soil profiles in Plot P1 seemed typical of Pseudotsuga-Polystichum sites developed under fairly wet climatic conditions. Despite the relatively high rainfall of this section of the valley, $\mathrm{A}_{2}$ horizons consisted of merely one or two centimeter's of reddish-brown sandy loam (Table VIII, Fig. 13 F). The occurrence of mottling in all horizons indicated that scepage moisture was influential even in surface layers, a characteristic of beta-gley soils (46). In addition to augmenting soil-moisture supply, this seepage presumably ameliorated the leaching action of the regional climate and limited the development of an $A_{2}$ horizon. The clay-loam layer at the base of profiles limited the downward percolation of seepage moisture. Profiles in this plot were comparable to the Stamp series of Day, Farstad, and Laird (10).

The Deadwood Creek Plot (P4), on stratified outwash material in the bottom of a wide U-shaped valley, represented Pseudotsuga-Polystichum stands growing on fertile soils under fairly dry climatic conditions. The presence of a black, loamy, $\mathrm{A}_{1}$ horizon with a crumb structure, indicated the relatively high fertility of the soil in this plot. Although some bleaching was present beneath piles of decayed wood, no $\mathrm{A}_{2}$ horizon had developed. Profiles in this plot were similar to the Qualicum series, although their predominantly yellowish-brown coloration showed that they also had an affinity with the Somas series (10).

Soil profiles in Plots P2 and P5 were characteristic of soil development under the influence of a high water table. Profiles in Plot P5 (Table VIII, Fig. 13 I) resembled the Puntledge series of Day, Farstad, and Laird (10) in being comprised of a shallow loamy deposit overlaying an impervious gleyed layer which caused a high water table. In Plot P2 (Table VIII, Fig. $13 \mathrm{H}$ ), the presence of fine gravel and shotty concretions seemed to render the profile above the ortstein quite porous. No evidence of the gray coloration usually associated with gleization was apparent. This lack of gray color was attributed to the ease with which ground water moved through the profile, a feature these soils had in common with the Bowser series (10).

Soil profiles in Plot P3 (Table VIII, Fig. 13 G) were typical of soil derelopment on fertile alluvial benches. Most profiles included an $\Lambda_{1}$ horizon, and $\Lambda_{2}$ horizons occurred only beneath piles of decayed wood. The B horizon, which intergraded from a very dark-brown loam to a yellowish-brown sandy loam, contained almost no material larger than $2 \mathrm{~mm}$. in diameter. The soils of this plot corresponded to the Chemainus series (10). 
The Thuja-Lysichitum plots occurred where seepage water came to the surface and the resulting streamlets spread out to form swamps. That such swamps were not stagnant and infertile was shown by their circumneutral pH ralues (Table VIII) and the slowly moving water which trickled through them. Profiles within the swampy areas consisted of varying depth of black muck, underlain by an olive gray, gleyed, sandy-loam layer (Fig. $13 \mathrm{~J}$ ). Before exposure to the air, bluish mottling was predominant, indicating that this layer wis continuously submerged. Tree roots were not encountered in the swampy parts of these plots.

Swampy areas were bordered by hummocks and banks of partially decomposed litter and wood, underlain by layers of very dark-brown, felty mor and reddish-brown, fibrous peat. These layers were densely interwoven by Thuja plicata roots. Tree roots, however, rarely extended into the gleyed, gravelly material below the peaty layers.

\section{Moisture Regimes}

Weather patterns during the soil-moisture sampling period were generally in accord with average values, although some variation did occur (Appendix II). In 1951, for example, below-average rainfall from April until the end of August accentuated the normal summer drought. At Cassidy, 12 miles to the east of Deadwood Creek, rainfall during this period was only 2.88 in. compared with an average of $10.75 \mathrm{in}$. Even Nitinat Camp in the central mountainous region recorded only $6.60 \mathrm{in}$., compared with the $16.44 \mathrm{in}$. normally received. In 1952, the summer drought period extended into autumn longer than usual. Rainfall at Deadwood Creek during September and October was only 1.3 in. as compared with the $7.5 \mathrm{in}$. average, and only $5.0 \mathrm{in}$. were recorded at Fourth Lake, considerably less than the average of $16.4 \mathrm{in}$. for this period.

During the winter of 1952-53 temperatures were more moderate than usual and most precipitation fell as rain. Precipitation during December and January was unusually heavy. Deadwood Creek received 31.9 in. during this period compared with an average of 20.6 in. and the Echo Mountain value of 47.8 in. was 18 in. more than usual. Precipitation was also somewhat greater than normal during June and July, 1953, and temperatures were below average.

\section{Pseudotsuga-Gaultheria-Peltigera association}

Depletion of soil-moisture reserves in the Pseudotsuga-Gaultheria-Peltigera plots began in May and continued throughout the growing season (Table IX, Fig. 14). In 1951 and 1952, available soil moisture in the Wolf Mountain, Deadwood Creek, and Valley Plots was reduced to very small amounts at all depths by early July. Although the summer of 1953 was somewhat wetter than usual, low percentages were still recorded by August.

Soil-moisture deficits in the Fourth Lake Plot (L1) were less marked than they were in the easterly plots, even during the dry summers of 1951 and 1952. Appreciable reduction in soil-moisture content had not occurred below the surface layers of the relatively fine-textured profile sampled in Plot L1 during early September, 1952, although values were quite low in the coarse-textured profile sampled during August. Even litter layers in the westerly plots had shorter periods of desiccation than in the stands farther east. It was noted, for example, that early September rainfall in 1951 was sufficient to re-wet litter layers in the Fourth Lake and Valley Plots, although these layers in the Deadwood Creek and Wolf Mountain Plots remained below wilting percentage until the end of the month. 


\section{Pseudotsuga-Gaultheria association}

Available soil moisture was not depleted as early in the growing season in the Pseudotsuga-Gaultheria plots as it was in the Pseudotsuga-GaultheriaPeltigera stands. Marked reductions were nevertheless evident by July and amounts of available moisture remained small until replenished by the autumnal rains. As in the Pseudotsuga-Gaultheria-Peltigera association, litter layers remained below wilting percentage during much of the growing season. Such desiccation presumably decreased the effectiveness of summer rainfall, since litter layers might absorb nearly $1 \mathrm{~cm}$. of water before horizons containing appreciable numbers of plant roots were re-wet.

The importance of vegetation in moisture depletion was evident from the data for Plots G4 and G6 (Table IX). During the autumn of 1952 these plots were swept by ground fires which removed all the subordinate vegetation and, on Plot G6, killed some trees. In 1953, moisture deficits in these disturbed plots were small, even though soil-moisture depletion had been as great as it was in other plots of the Pseudotsuga-Gaultheria association (Plots G3 and G5) during the previous growing season.

After periods of heavy rainfall in late autumn, temporary water tables were common above hardpan layers and high moisture percentages were recorded at all depths. When Plot G6 was sampled in early December, 1951, this perched water table above the ortstein layer was $10 \mathrm{~cm}$. deep. On resampling in early January, 1952, some two weeks after a heavy snowfall had covered the ground, there was no gravitational water above the hardpan and the moisture percentage of all layers was lower. Presumably, such a reduction in moisture content occurred because internal drainage in these gravelly, shotty soils was rapid, and excess moisture quickly drained away once further addition of water was prevented by the snow blanket. During the winter of 1952-53, perched water tables were present more or less continuously since there was little snow or frozen soil to restrict infiltration of the abnormally heavy rainfall of December and January.

\section{Pseudotsuga-Tsuga-Gaultheria association}

Soil-moisture values in the Pseudotsuga-Tsuga-Gaultheria plots were high in the available range during the early part of the growing season. Even litter layers contained available moisture at most samplings. Low values, particularly in the upper $20 \mathrm{~cm}$., were recorded in July, 1951, and August, 1952, although periods with small amounts of available soil moisture were of shorter duration than in the droughty Pseudotsuga-Gaultheria plots.

\section{Pseudotsuga-Tsuga-Hylocomium association}

Available soil moisture was virtually exhausted from the surface layers of the easterly subassociation typicum plots of the Pseudotsuga-T'suga-Hylocomium association during the late summer of 1951 and 1952. The moisture content of deeper layers was also reduced to small amounts by the latter part of these growing seasons. This depletion was particularly marked in Plot M4 (Table IX). On the other hand, deficiencies were of shorter duration in Plot M1 at Fourth Lake, where even litter layers contained available moisture at most samplings. Litter layers in the easterly plots remained below wilting percentage much of the growing season. Soil-moisture deficits were smaller in all plots during the wet summer of 195.3 than they were during 1951 and 1952.

Soil-moisture deficits in the subassociation nudum plots of the PseudotsugaTsuga-Hylocomium association were smaller than in the typicum plots. Low values were nevertheless recorded in surface layers by the end of the unusually 


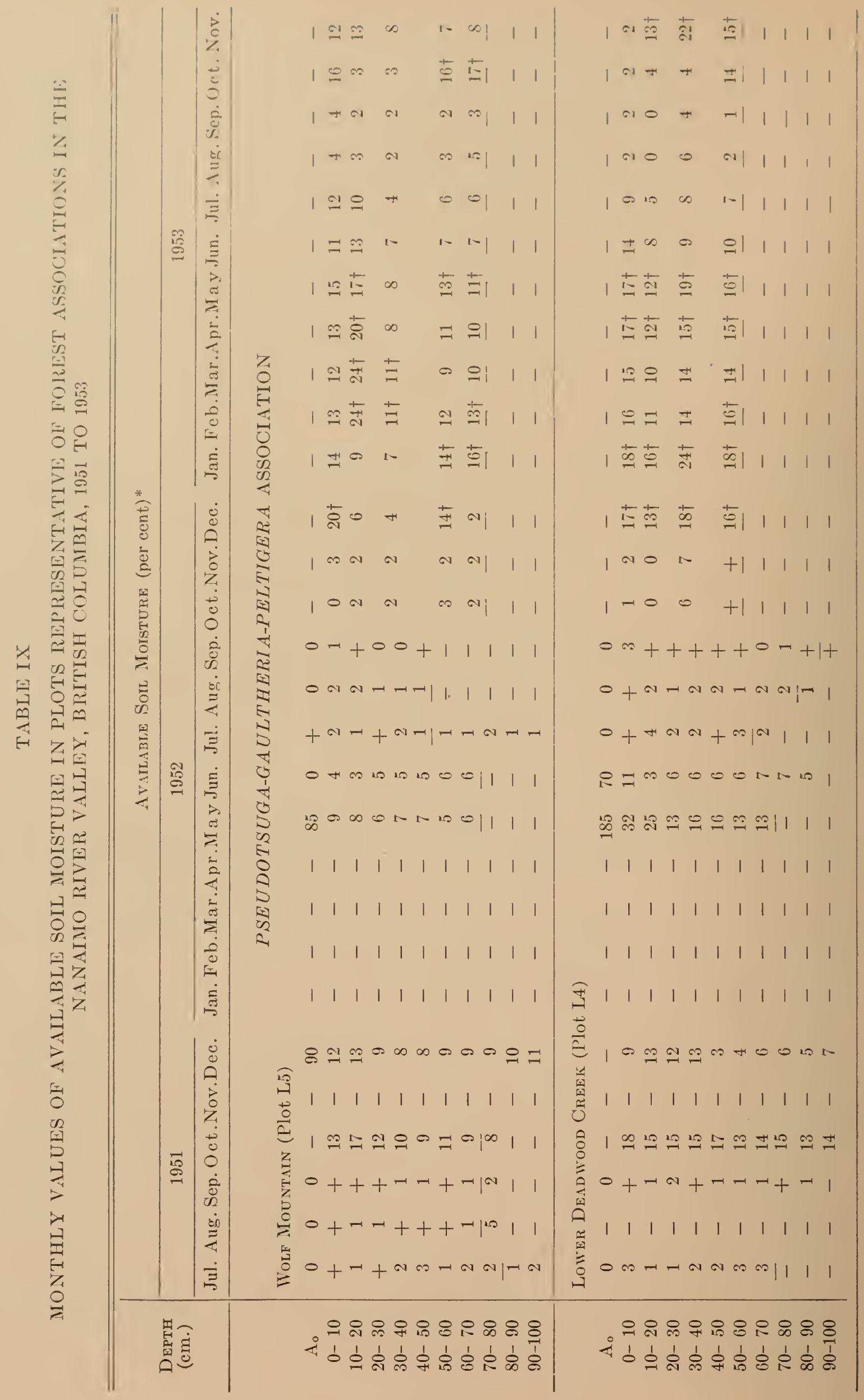




\begin{tabular}{|c|c|c|c|c|c|c|c|c|c|c|c|}
\hline 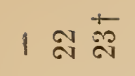 & $\stackrel{\infty}{\rightarrow}$ & 焉 1 & 11 & & & । 苛京 & & 兽|1 1 & 11 & & 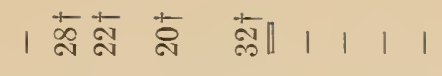 \\
\hline 1 N & 品 & \pm & 11 & । & & 1 बें & $H$ & $20|1|$ & 11 & & $\begin{array}{lllll}+ & 1 & 1 & 1 & 1\end{array}$ \\
\hline $1+0$ & -1 & $\infty 1$ & 11 & 1 & & 12020 & a & $\infty|1|$ & 11 & & 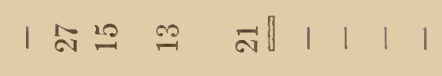 \\
\hline $10 n$ & $\neg$ & $\infty 1$ & 11 & 1 & & $1+0$ & $H$ & $0 \mid 11$ & 11 & & 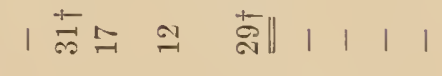 \\
\hline 1 으 & $\Rightarrow$ & -11 & 11 & 1 & & $1 \stackrel{\infty}{\sim}$ & $\infty$ & $\infty|1|$ & 11 & & 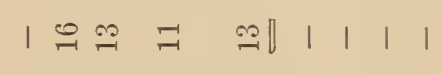 \\
\hline $1 \stackrel{20}{-1}=$ & $\stackrel{\sim}{\sim}$ & $\infty 1$ & 11 & 1 & & $1 \stackrel{ }{\mathcal{I}}$ & 우 & $\theta|1|$ & । 1 & & 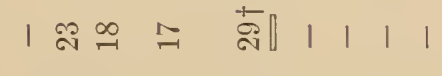 \\
\hline $1+\frac{1}{\alpha} \vec{N}$ & 19 & 인 & 11 & । & & $1 ニ シ ゙$ & $\stackrel{+}{\mathcal{I}}$ & $\stackrel{\simeq}{*}|| \mid$ & 11 & & 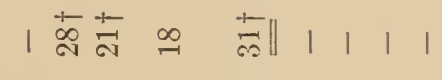 \\
\hline 1 สิ จิ & $\Xi$ & $\infty 1$ & 11 & 1 & & । $\stackrel{\infty}{二}$ & $\stackrel{+}{\Im}$ & $\cong 111$ & 11 & & 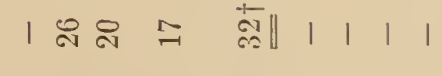 \\
\hline 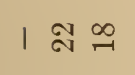 & $\stackrel{9}{\rightarrow}$ & $\infty 1$ & 11 & 1 & & $1 \stackrel{\infty}{\oplus}$ & 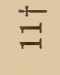 & $\approx 111$ & 11 & & 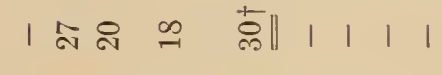 \\
\hline | & $\approx$ & $=11$ & 11 & 1 & & 1 啇望 & $\stackrel{+}{ \pm}$ & \pm 111 & 11 & & | \\
\hline $1+\frac{1}{\pi}$ & 志 & $\Rightarrow 11$ & 11 & 1 & & 1 节志 & $\stackrel{4}{10}$ & 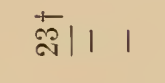 & 11 & & 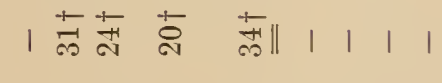 \\
\hline 1 志高 & $\stackrel{+}{\mathbb{N}}$ & $\stackrel{+1}{+1}$ & 11 & । & & 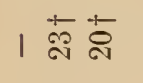 & $\stackrel{+}{\stackrel{+}{\oplus}}$ & 部|1 1 & 11 & & | \& \\
\hline | 10 จิ & 产 & $\infty 1$ & 11 & 1 & & । 䓞动 & 0 & $20|1|$ & 11 & & | \\
\hline I $\infty$ & - & $\infty 1$ & 11 & & & 12020 & 20 & $H|1|$ & 11 & & | \\
\hline$a c$ & $+-r$ & +-11 & -1 & । & & & $\infty$ & $+\infty x_{\infty}=0$ & 11 & & 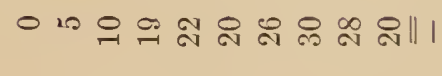 \\
\hline & $++\rightarrow$ & $-1+$ & $-\left.\infty\right|_{1} ^{\circ}$ & & & & -- & $\begin{array}{rlll}-1 & 1 & 1\end{array}$ & । 1 & & $0004 h 400010001111$ \\
\hline & $\alpha \infty$ & 001 & $r p^{n}$ & & & $\operatorname{orat}$ & $-\infty$ & $\left.\infty\right|^{+\infty} \mid 1$ & 11 & & 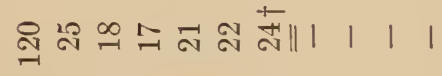 \\
\hline$\underset{\mathbb{T}}{\infty} \infty 20$ & 2000 & $\infty 201$ & +1 & 1 & & $\stackrel{\infty}{\rightarrow} \underset{-1}{=}=$ & $\Rightarrow 1$ & 0,00 . & $\approx \cong$ & & 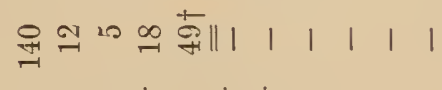 \\
\hline 원 $\stackrel{12}{129}$ & $\stackrel{9}{-}=$ & $=2211$ & 11 & 1 & & $\stackrel{8}{\circ}=$ & & $\begin{array}{llll}1 & 1 & 1 & 1\end{array}$ & 11 & & 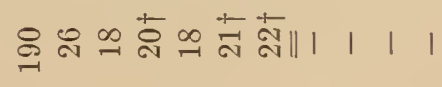 \\
\hline $1 \quad 11$ & $1 \quad 11$ & $1 \quad 11$ & 11 & 1 & & $1 \quad 1 \quad 1$ & 11 & $\begin{array}{llll}1 & 1 & 1 & 1\end{array}$ & 11 & & $\begin{array}{llllllllllll}1 & 1 & 1 & 1 & 1 & 1 & 1 & 1 & 1 & 1 & 1\end{array}$ \\
\hline $1 \quad 11$ & $1 \quad 11$ & $1 \quad 1 \quad 1$ & 11 & 1 & & $1 \quad 1 \quad 1$ & । 1 & $\begin{array}{llll}1 & 1 & 1 & 1\end{array}$ & । 1 & & $\begin{array}{llllllllllll}1 & 1 & 1 & 1 & 1 & 1 & 1 & 1 & 1 & 1 & 1\end{array}$ \\
\hline 111 & 111 & 111 & 11 & 1 & & 1111 & । 1 & $\begin{array}{llll}1 & 1 & 1 & 1\end{array}$ & 11 & & $\begin{array}{lllllllllll}1 & 1 & 1 & 1 & 1 & 1 & 1 & 1 & 1 & 1 & 1\end{array}$ \\
\hline $\begin{array}{lll}1 & 1 & 1\end{array}$ & 111 & 111 & 11 & । & & $\begin{array}{lll}1 & 1 & 1\end{array}$ & । 1 & $\begin{array}{llll}1 & 1 & 1 & 1\end{array}$ & 11 & & $\begin{array}{lllllllllll}1 & 1 & 1 & 1 & 1 & 1 & 1 & 1 & 1 & 1 & 1\end{array}$ \\
\hline 光 & $\exists=\infty$ & 1000 & $\infty 11$ & 1 & & 웡휴 & 落 & 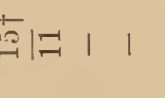 & । 1 & & 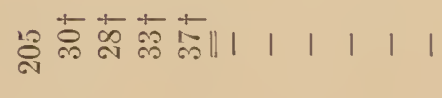 \\
\hline 111 & 111 & 111 & 11 & 1 & 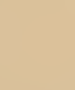 & $\begin{array}{lll}1 & 1 & 1\end{array}$ & 11 & $\begin{array}{llll}1 & 1 & 1 & 1\end{array}$ & 11 & $\widehat{\exists}$ & $\begin{array}{lllllllllll}1 & 1 & 1 & 1 & 1 & 1 & 1 & 1 & 1 & 1 & 1\end{array}$ \\
\hline & & $\approx: 90$ & $\infty \infty$ & & $\widehat{\widehat{I}}$ & 空果志吉 & & $\omega_{1}^{\infty} \infty$ & । 1 & $\stackrel{0}{E}$ & 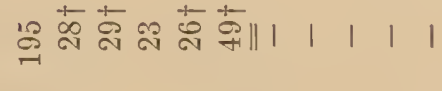 \\
\hline & $-r-$ & $\neg|\neg|$ & 11 & & $\stackrel{\overrightarrow{0}}{\varrho}$ & $\because+$ & & $+4 \mid a n$ & 11 & 量 & 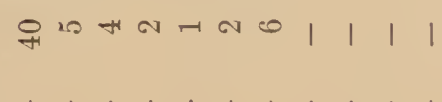 \\
\hline $\begin{array}{ll}0 & a \\
1 & 1\end{array}$ & $\begin{array}{ccc}1 & 1 \\
-1 & -1 & -1\end{array}$ & & 11 & 1 & 总 & 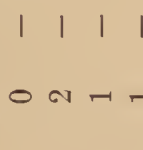 & & 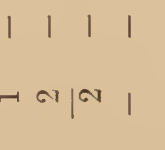 & $\begin{array}{ll}1 & 1 \\
1 & 1\end{array}$ & 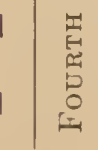 & 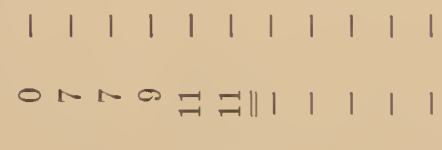 \\
\hline $\begin{array}{ll}0 & 8 \\
0 & 8 \\
1 & 1 \\
0 & 1 \\
-1\end{array}$ & 유 용 & 808 & $8 \&$ & & & 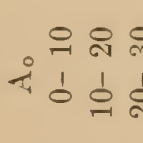 & & $\begin{array}{l}8 ㅇ ㅛ \\
110 \\
10 \\
10\end{array}$ & $\stackrel{1}{\infty}$ & & 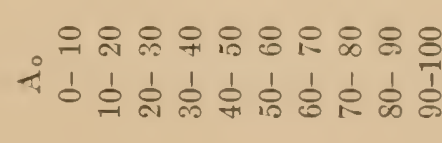 \\
\hline
\end{tabular}




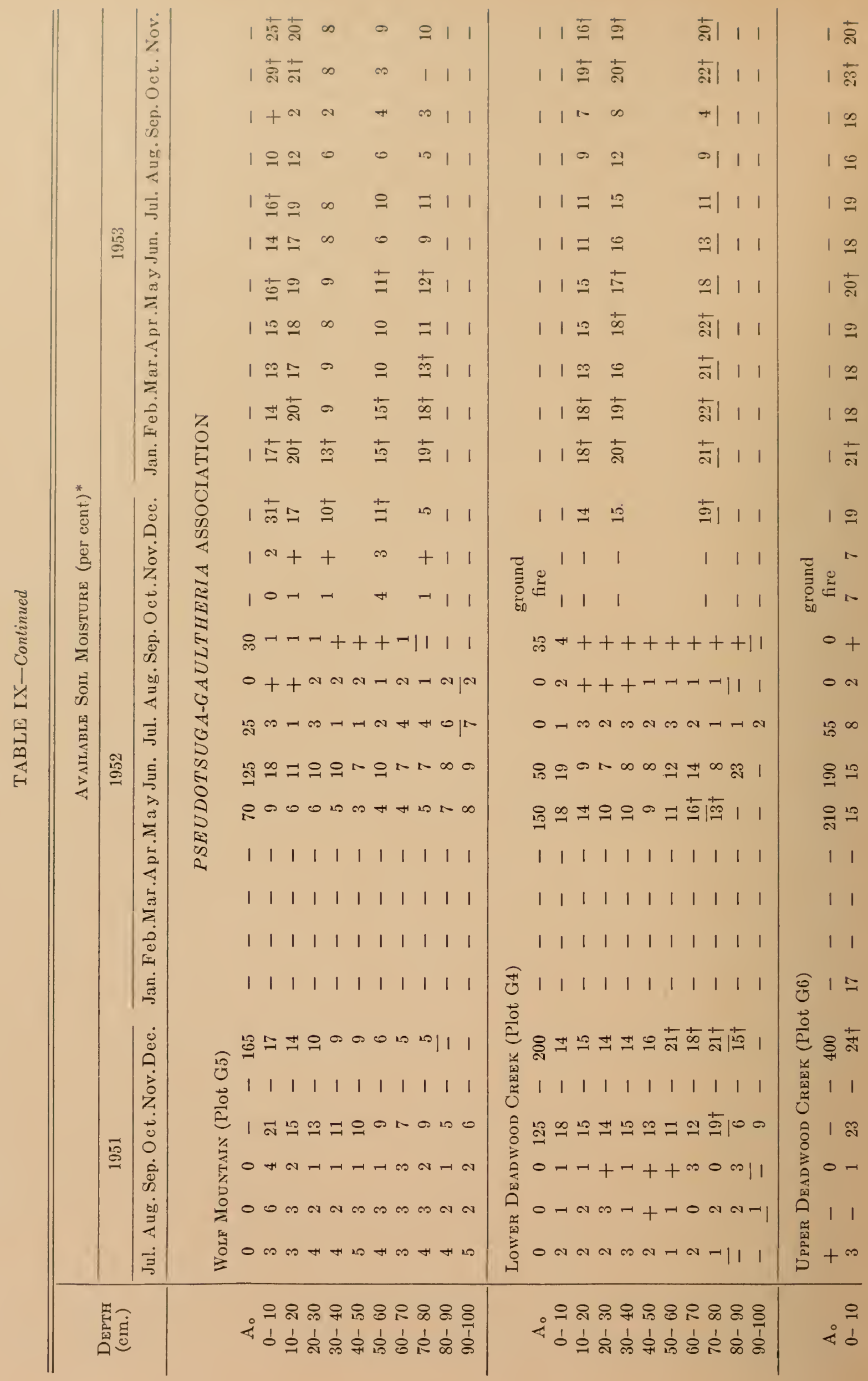




\begin{tabular}{|c|c|c|c|c|c|c|c|c|c|c|c|c|c|}
\hline \pm & & $\Rightarrow 1111$ & & 1 命 & 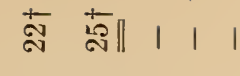 & 11 & & । ๙ ส & $\stackrel{+0}{-9}$ & & $\stackrel{9}{9}$ & Ell & | 1 \\
\hline$\vec{A}$ & $\stackrel{9}{\sim}$ & $\stackrel{ \pm}{ \pm} \mid \begin{array}{lll}\mid & 1 & 1\end{array}$ & & 100 & 亩 芯\| | | | & 11 & & 1 总电 & $\neg$ & & $=$ & $\stackrel{\infty}{\infty} \| 1$ & 11 \\
\hline$\stackrel{29}{7}$ & 옥 & 이 1111 & & $1 \infty-$ & 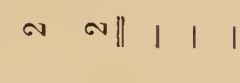 & 11 & & $1 \bumpeq \stackrel{\infty}{二}$ & $\stackrel{+}{-}$ & & $\stackrel{25}{-2}$ & 데 1 & 11 \\
\hline$\dddot{q}$ & $\exists$ & 인 1111 & & $1 \stackrel{-1}{=}$ & 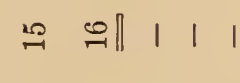 & 11 & & $10=$ & $\stackrel{-}{-}$ & & 오 & & 11 \\
\hline$\stackrel{0}{\sim}$ & $\Rightarrow$ & 윗 111 & & $1 \bumpeq \cong$ & $\Rightarrow \approx \| \quad|\quad|$ & 11 & & $1 \infty \#$ & $\varrho$ & & $\stackrel{ }{-}$ & $2 \| 1$ & 11 \\
\hline$\stackrel{9}{-1}$ & $\stackrel{2}{-1}$ & 이 111 & & $1 \bumpeq \vec{\sim}$ & $\triangleq \vec{\sigma} \| \mid \begin{array}{lll}\mid & \end{array}$ & 11 & & | $\stackrel{\infty}{\rightarrow}$ & $\stackrel{+}{\sim}$ & & $\cong$ & $\underline{\underline{\theta}} \|$ & 11 \\
\hline$\stackrel{\oplus}{\circ}$ & $\stackrel{2}{\sim}$ & $\Rightarrow \begin{array}{lll}1 & 1 & 1\end{array}$ & & 1 กิ ลิ & $\stackrel{ \pm}{ \pm} \approx \| \quad|\quad|$ & 11 & & | 志禺 & $\stackrel{+}{\beth}$ & & 茫 & 节 1 & 11 \\
\hline 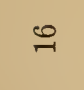 & $\stackrel{\Re}{\rightarrow}$ & $=1 \begin{array}{lll}1 & 1 & 1\end{array}$ & & 1 志志 & 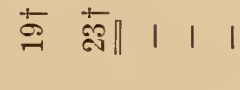 & 11 & & $1 \stackrel{2}{a}$ & $\approx$ & & 䔽 & 节 । & 1 \\
\hline 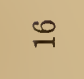 & 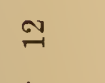 & 익 1 | 1 & & $1 \stackrel{2}{-12}$ & 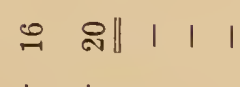 & 11 & & 1 ตั ลำ & $\exists$ & & 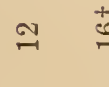 & & । 1 \\
\hline - & $\stackrel{+}{ \pm}$ & $\Rightarrow \begin{array}{llll}1 & 1 & 1 & 1\end{array}$ & & 1 芯离 & 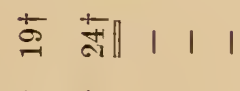 & 11 & $\stackrel{\circ}{\Theta}$ & & $\Rightarrow$ & & & sill & \\
\hline 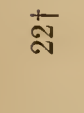 & సे & $\stackrel{+1}{-1} \mid \begin{array}{lll}1 & 1\end{array}$ & & 1 苍菜 & 芯 志国| | | & 11 & $\begin{array}{l}3 \\
0 \\
0\end{array}$ & | 志志 & $\stackrel{+}{9}$ & & $\stackrel{+}{=}$ & $\pm \| 1$ & । 1 \\
\hline$\stackrel{\oplus}{0}$ & 索 & $\Rightarrow \mid \begin{array}{lll}1 & 1 & 1\end{array}$ & & । 荡范 & 衣 & 11 & $\begin{array}{l}\pi_{4}^{2} \\
\square\end{array}$ & 1 苏落 & $\stackrel{+19}{-1}$ & & $\stackrel{+}{ \pm}$ & $=1$ & । 1 \\
\hline & & $\begin{array}{llll} & 1 & 1 & 1\end{array}$ & & 1 왹 0 & 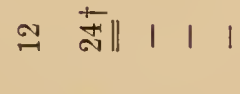 & 11 & 司 & | 高芯 & $\infty$ & & $\Rightarrow$ क & 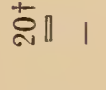 & । 1 \\
\hline & 20 & $\infty \begin{array}{llll}1 & 1 & 1 & 1\end{array}$ & & $1 \infty \infty$ & $\begin{array}{llllll}\infty & \infty & 1 & 1 & 1\end{array}$ & 11 & $\underset{N}{\mathbb{N}}$ & 100 & $\infty$ & & $\infty \quad \sigma$ & $\infty \| 1$ & । 1 \\
\hline++ & $-1-$ & $\sim-1+1$ & & 品A hN & $0-O N|| \mid$ & 11 & క్ & 路 & & & 9.9 & $\begin{array}{lll}1 & 1 & 1\end{array}$ & 11 \\
\hline & $\infty \sim$ & $\left.\infty \infty\right|^{-1} \mid$ & & 10000 & $A \rightarrow \infty$ & $\infty 1$ & $\begin{array}{c}\mathbb{1} \\
0 \\
0 \\
0\end{array}$ & OHNo & & & $H$ & 1 & 1 \\
\hline 10 & ON & $-100 / 001$ & & & $\infty N \| 1 \quad 1 \quad 1 \quad 1$ & 11 & & 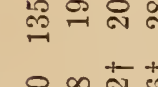 & & S & $5 \| 11$ & $\begin{array}{lll}1 & 1 & 1\end{array}$ & 11 \\
\hline$\approx \cong$ & 0 유: & $\underset{\sim}{\sim} \underset{\sim}{2} \infty|1|$ & & & 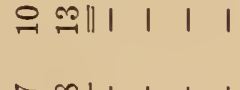 & & $\begin{array}{l}0^{4} \\
i_{0} \\
03\end{array}$ & $\infty^{\infty} \infty$ & & I & 111 & 11 & 11 \\
\hline$\stackrel{\infty}{\sim}=$ & $\exists=7$ & 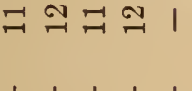 & & : & $-\infty \mid \begin{array}{llll}1 & 1 & 1 & 1\end{array}$ & 11 & ถู่ & & & & $\begin{array}{c}4 \| 1 \\
\end{array}$ & 11 & 1 \\
\hline & 11 & $\begin{array}{llllllll}1 & 1 & 1 & 1 & 1\end{array}$ & & $\begin{array}{llll}1 & 1 & 1 & 1\end{array}$ & $\begin{array}{llllll}1 & 1 & 1 & 1 & 1 & 1\end{array}$ & 11 & $\begin{array}{l}D_{1} \\
0=1 \\
012\end{array}$ & $\begin{array}{lll}1 & 1 & 1 \\
1 & 1 & 1\end{array}$ & 11 & 1 & 11 & 11 & 11 \\
\hline 11 & 11 & $\begin{array}{llll}1 & 1 & 1 & 1\end{array}$ & & $\begin{array}{lll}1 & 1 & 1\end{array}$ & $\begin{array}{lllllllllll}1 & 1 & 1 & 1 & 1 & 1\end{array}$ & 11 & 2 & $\begin{array}{lll}1 & 1 & 1 \\
-1 & 1\end{array}$ & 1 & 1 & 11 & 11 & 11 \\
\hline 11 & 11 & $\begin{array}{lllll}1 & 1 & 1 & 1 & 1\end{array}$ & & $1 \quad 1 \quad 1 \quad 1$ & $\begin{array}{llllll}1 & 1 & 1 & 1 & 1 & 1\end{array}$ & & & 111 & 1 & I & 11 & & \\
\hline$=\approx$ & & 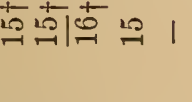 & & $\begin{array}{llll}1 & 1 & 1 & 1\end{array}$ & $\begin{array}{llllll}1 & 1 & 1 & 1 & 1 & 1\end{array}$ & 11 & & $\begin{array}{lll}1 & 1 & 1\end{array}$ & 11 & I & 11 & 11 & 1 1 \\
\hline 穴亦 & 苯京: & $\cong \stackrel{5}{a}|1| 1$ & & 落落志 & 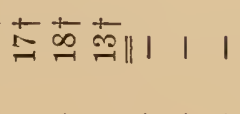 & 11 & & 1 㝵的告 & & & 용 & & 它京\| \\
\hline 11 & 11 & $\begin{array}{lllll}1 & 1 & 1 & 1 & 1\end{array}$ & & $\begin{array}{llll}1 & 1 & 1 & 1\end{array}$ & $\begin{array}{llllll}1 & 1 & 1 & 1 & 1 & 1\end{array}$ & 11 & & $\begin{array}{lll}1 & 1 & 1\end{array}$ & $\begin{array}{l}11 \\
++\end{array}$ & 1 & 11 & I 1 & \\
\hline$\approx$ & $\therefore \approx=$ & 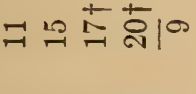 & $\widehat{\mathscr{O}}$ & 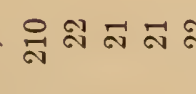 & 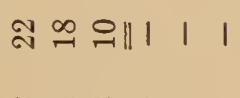 & 11 & $\stackrel{0}{2}$ & & & & & & 11 \\
\hline$\infty$ or & $\infty \infty$ & $\neg \sigma \propto N j^{N} \propto$ & $\vec{\Xi}$ & 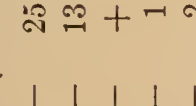 & $\begin{array}{llll}1 & 1\end{array}$ & $\begin{array}{ll}1 & 1 \\
1 & 1\end{array}$ & $\underset{3}{\stackrel{4}{4}}$ & 1 워 & & & $\begin{array}{llll}0 & 0\end{array}$ & & 11 \\
\hline $\begin{array}{ll}4 & 1 \\
4 & 1 \\
1 & 1\end{array}$ & 11 & 111111 & 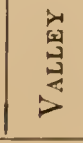 & 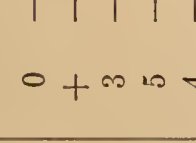 & 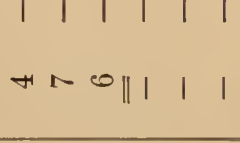 & $\begin{array}{ll}1 & 1 \\
1 & 1 \\
\end{array}$ & 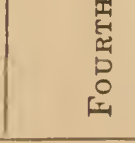 & $\begin{array}{cc}1 & 1 \\
20 & +\infty\end{array}$ & $\stackrel{1}{*}=$ & & 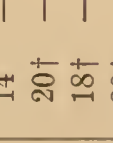 & & S \\
\hline $\begin{array}{ll}10 \\
1 \\
9\end{array}$ & 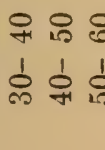 & 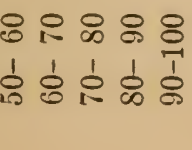 & & 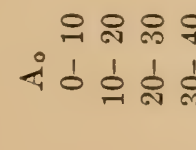 & 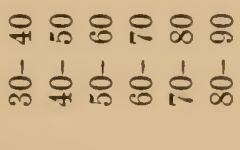 & $\begin{array}{ll}8 & 8 \\
1 & 0 \\
1 & 1 \\
\infty & 8\end{array}$ & & 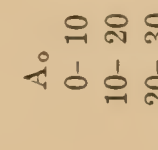 & & & $\therefore$ & : & \\
\hline
\end{tabular}




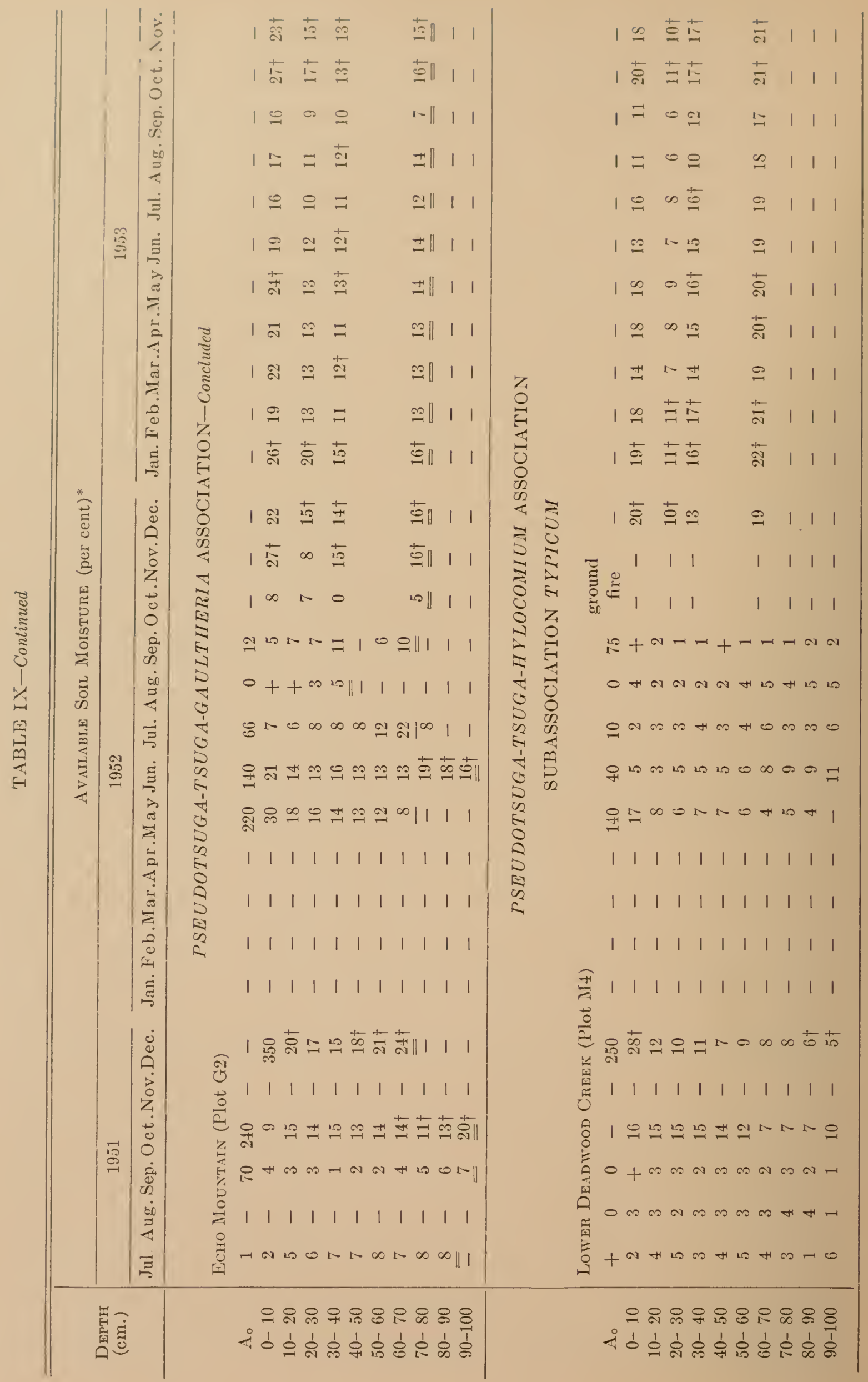




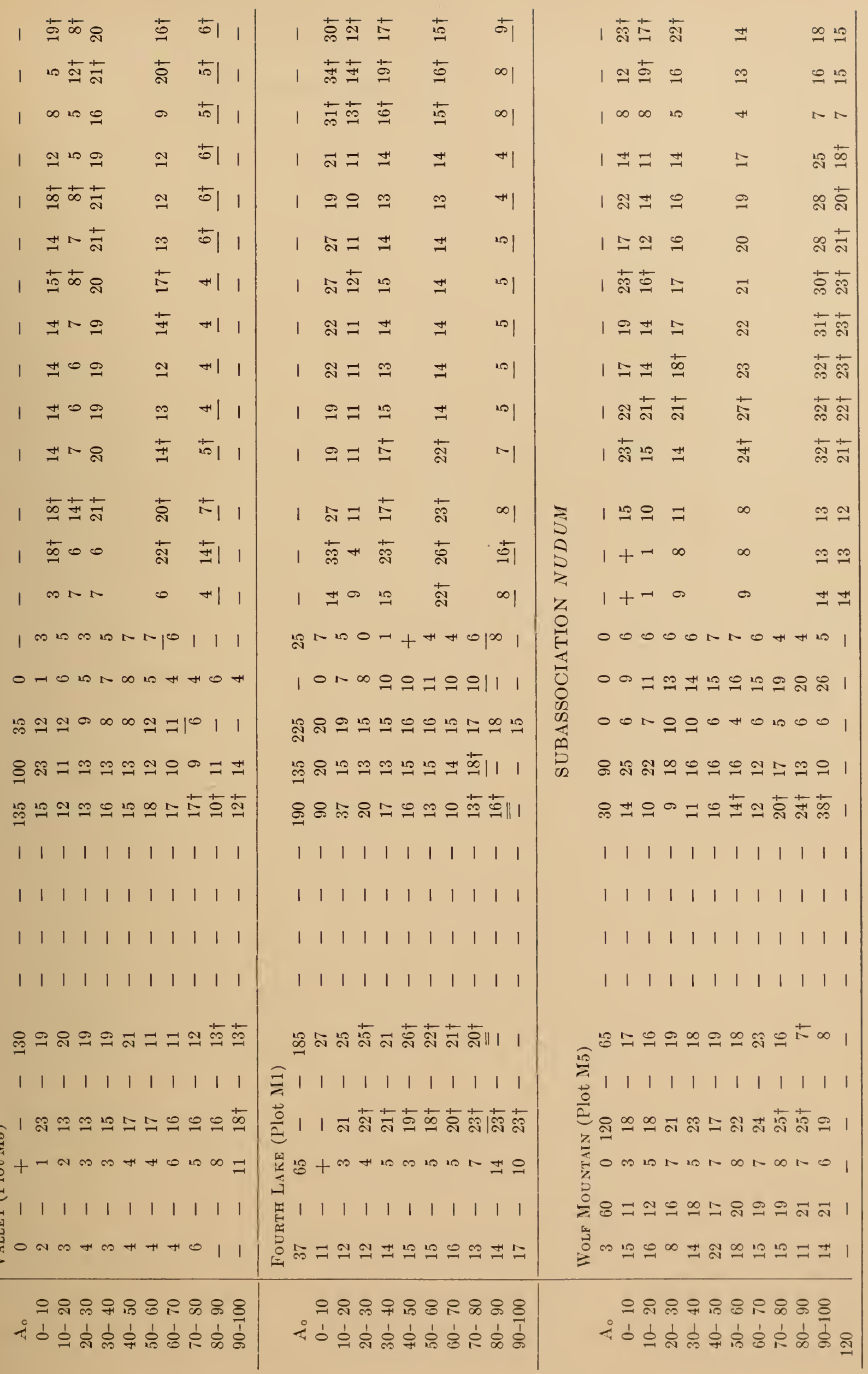




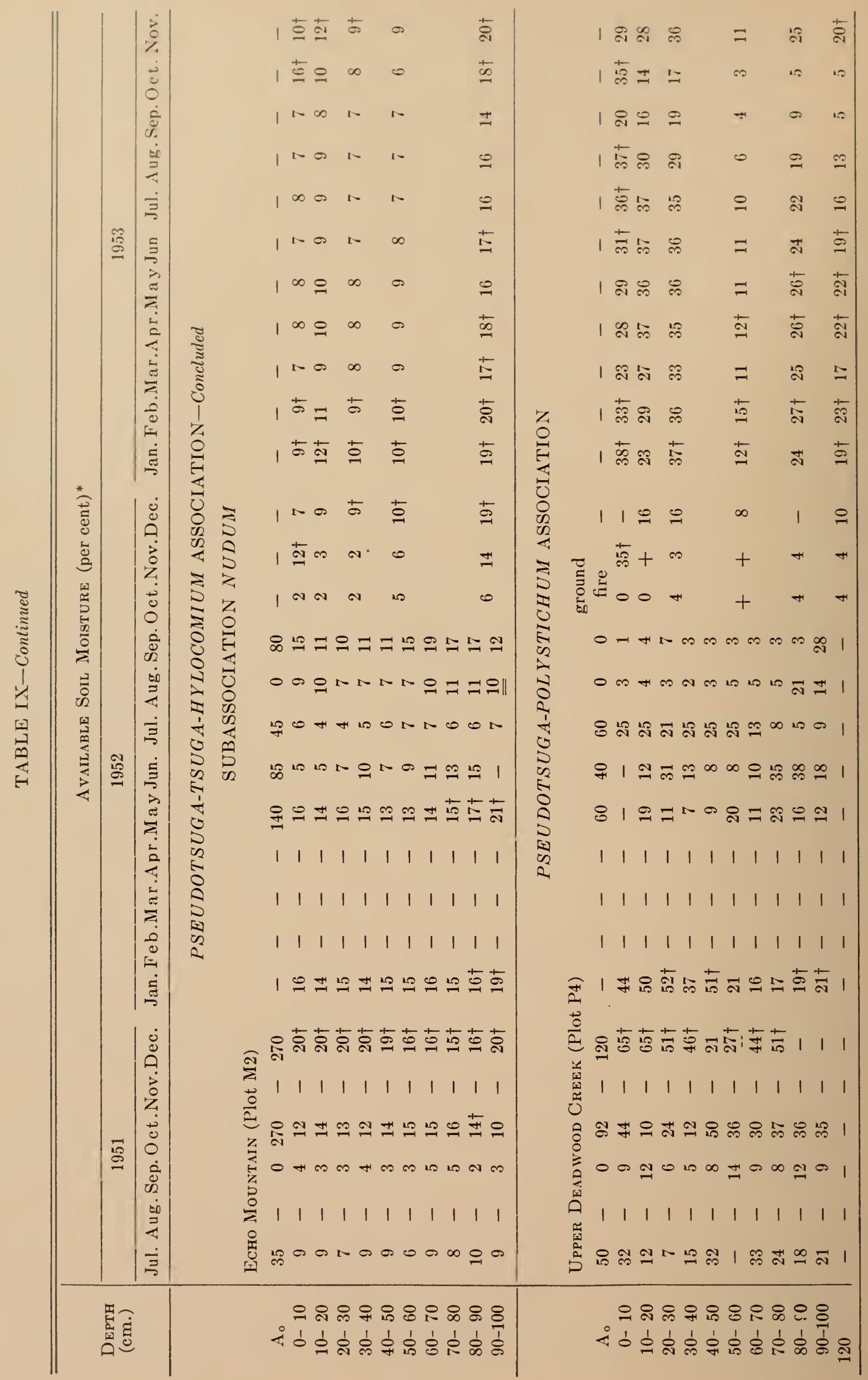




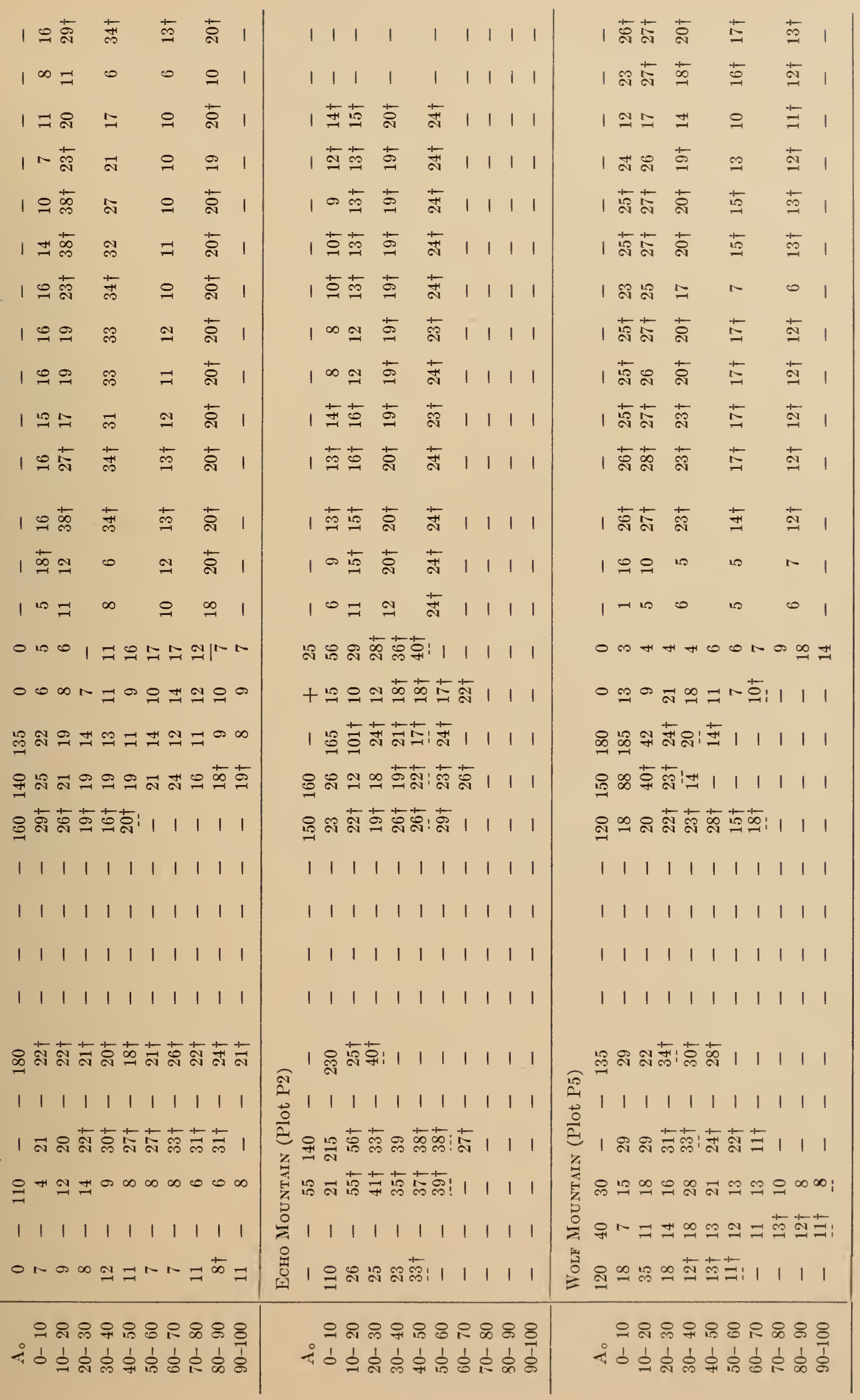




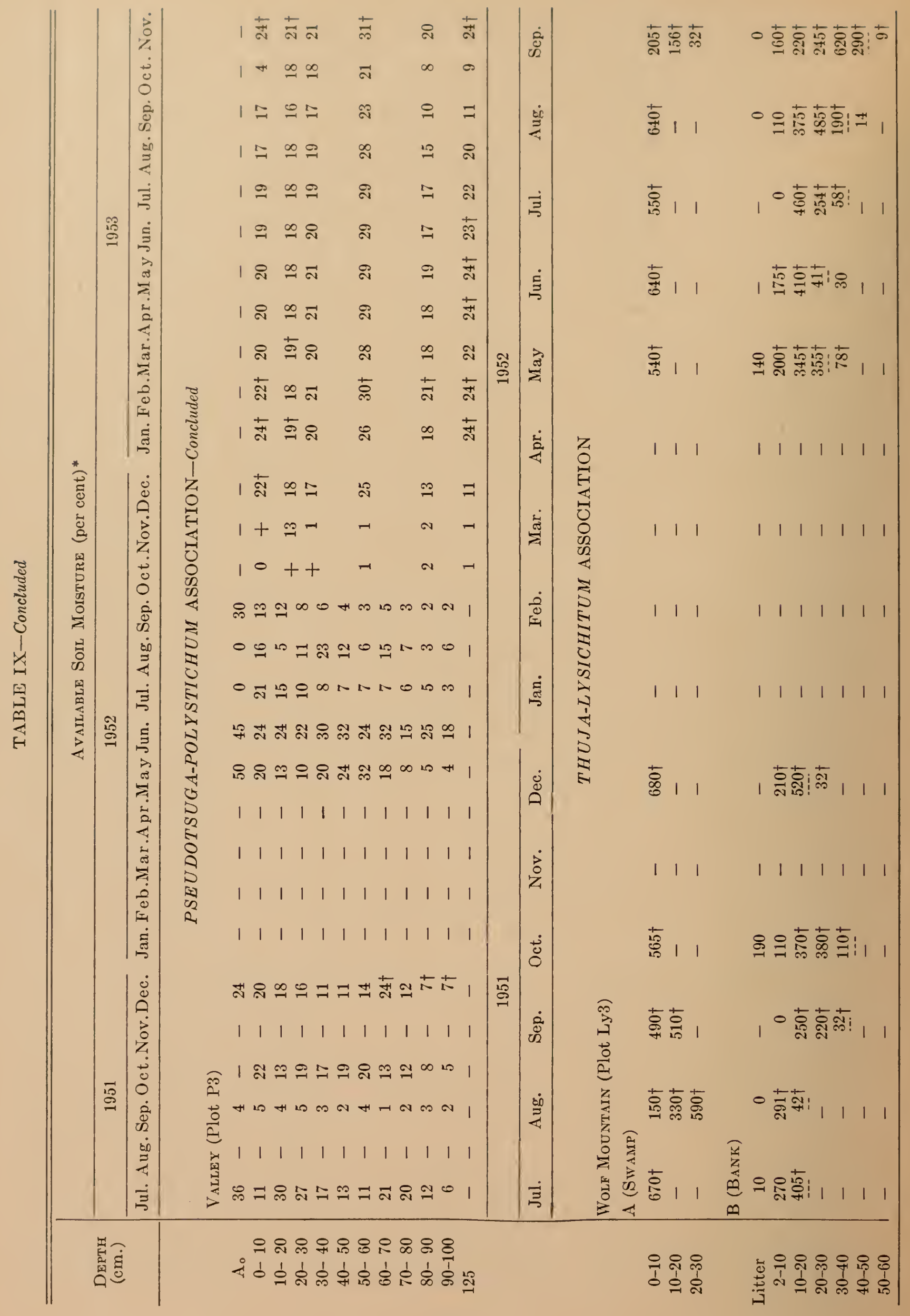




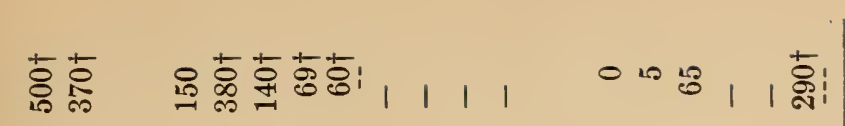

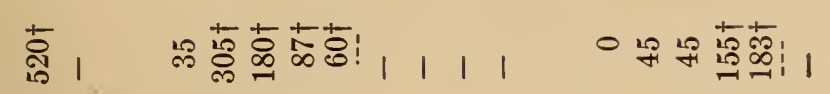

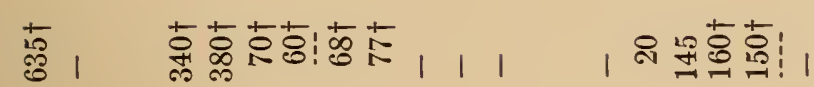

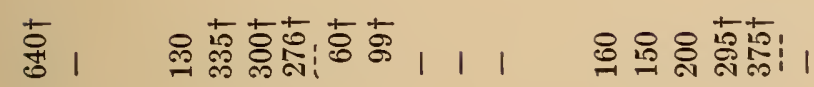

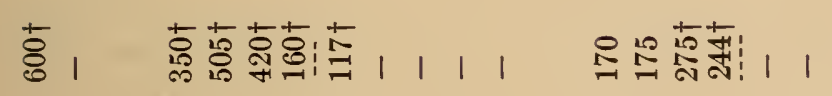

$\begin{array}{lllllllllllllllll}1 & 1 & 1 & 1 & 1 & 1 & 1 & 1 & 1 & 1 & 1 & 1 & 1 & 1 & 1 & 1 & 1\end{array}$

$\begin{array}{lllllllllllllllll}1 & 1 & 1 & 1 & 1 & 1 & 1 & 1 & 1 & 1 & 1 & 1 & 1 & 1 & 1 & 1 & 1\end{array}$

$\begin{array}{lllllllllllllllll}1 & 1 & 1 & 1 & 1 & 1 & 1 & 1 & 1 & 1 & 1 & 1 & 1 & 1 & 1 & 1 & 1\end{array}$

$\begin{array}{lllllllllllllllll}1 & 1 & 1 & 1 & 1 & 1 & 1 & 1 & 1 & 1 & 1 & 1 & 1 & 1 & 1 & 1\end{array}$

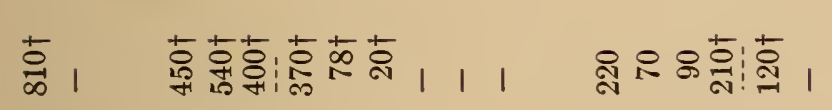

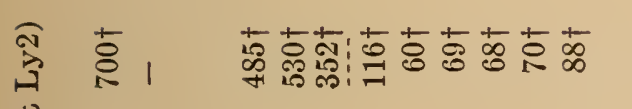

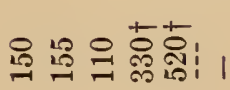

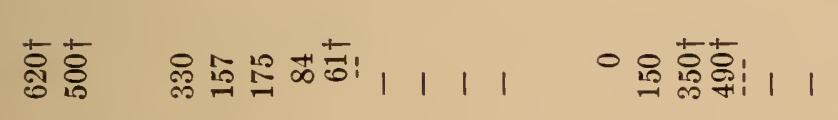

列

1

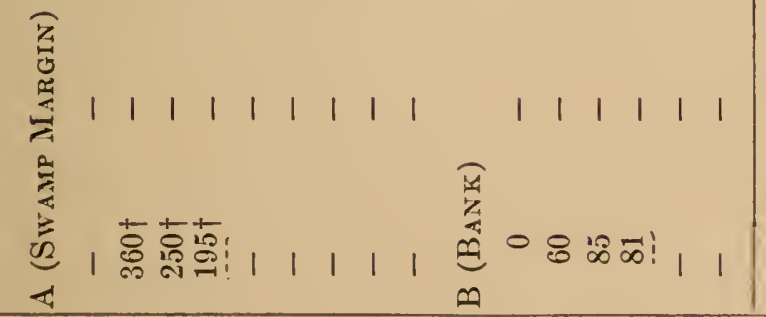

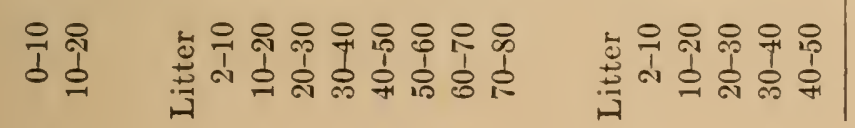

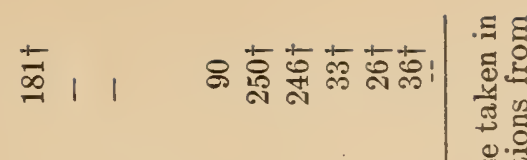

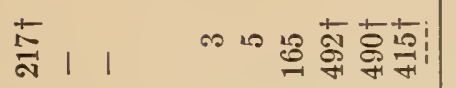

范 | | \&

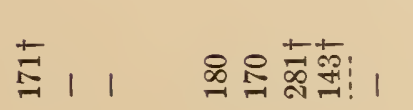

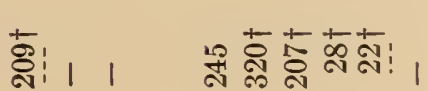

11

11

1 111

111

1 1

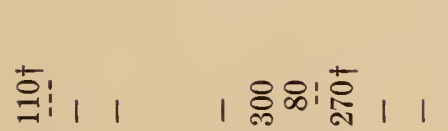

1 1 1

111111

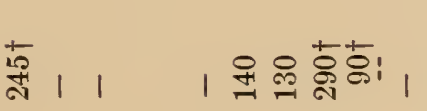

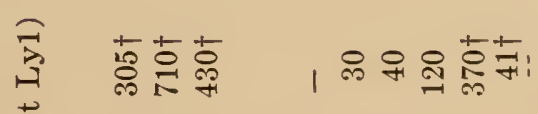

है

总

点

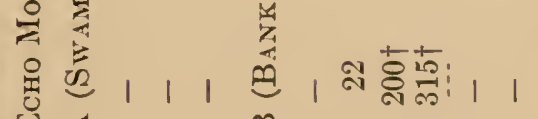

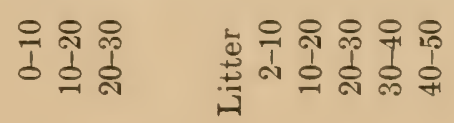




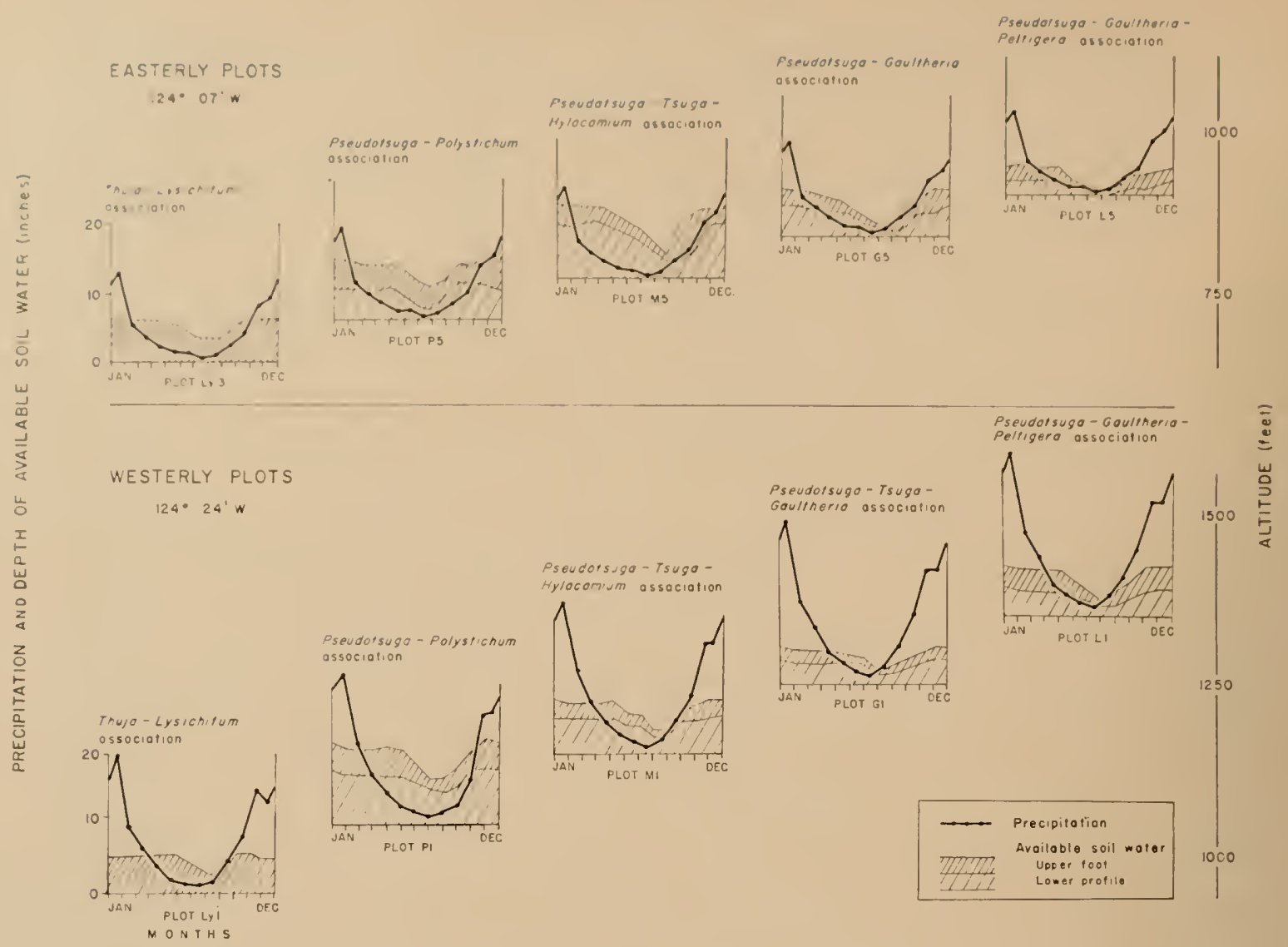

FIGURE 14-Average monthly precipitation and average depth of available soil water in plots representative of forest associations in the Nanaimo River Valley, British Columbia, 1951-1953. The moisture storage capacities of profiles may be judged by comparing the depths of available water present when soils are close to field capacity during winter months

prolonged summer drought of 1952. In the Echo Mountain Plot, gravitational water was present above the clay-loam layer during spring and autumn, although none occurred during the summer. Similarly, when this plot was sampled in early January, 1952, two weeks after heavy snowfalls had restricted further infiltration, no gravitational water was found above the clay-loam layer. Accumulations of water did occur in an open observation pit on Plot M5 after the prolonged autumnal rains of 1951, but normally the soil of this plot was well drained to a depth of $1 \mathrm{~m}$. or more.

\section{Pseudotsuga-Polystichum association}

Considerable depths of available moisture were present in most of the Pseudotsuga-Polystichum plots at all seasons and low moisture percentages were infrequent (Table IX). Seepage promoted such moist conditions in several plots.

In the Echo Mountain Plot (P2), a high water table was sustained by moisture seeping into the concave portion of the slope on which the plot was located. During spring and early summer, this seepage originated from the melting snow on a slope which reached nearly $3,000 \mathrm{ft}$. above sea level. The water table in an observation pit showed only moderate fluctuations in level. During much of the year, this level remained within $40 \mathrm{~cm}$. of the soil surface, although in the late summer of 1952 it dropped to $50 \mathrm{~cm}$. and during the winter it sometimes rose to within $20 \mathrm{~cm}$. of the ground line. While such levels represented conditions in a moist part of the stand, since the observation pit was near 
the margin of a slight depression, soil-moisture samples taken from other locations showed that values remained high in the available range throughout the growing season. Even litter layers were above wilting percentage at most samplings.

Fluctuations in the height of the water table in the observation pit on the Wolf Mountain Plot (P5) were greater than in the Echo Mountain Plot. The dry summer climate of the easterly portion of the valley in which Plot P5 was located and the short duration of the snow pack on the adjacent 1,600-ft. slope may account for this difference. During the latter part of the summer the water level in the observation pit was commonly $100 \mathrm{~cm}$. below the soil surface. Although available moisture in upper soil horizons was not exhausted at such times, percentages were usually lower than in the Echo Mountain Plot. At other seasons the water table remained about $40 \mathrm{~cm}$. below ground line. During a prolonged rainy spell in December, 1952, the level in the pit rose to within $10 \mathrm{~cm}$. of the soil surface.

Except in late summer and early autumn, some water was present in an observation pit in the Fourth Lake Plot (P1) the year round. Normally this seepage moisture flowed over the compact soil layer toward the base of the profile, some $80 \mathrm{~cm}$. beneath the surface. In late spring and early summer, water from melted snow usually raised the water level and during prolonged wet spells, such as that of December, 1952, water even overflowed from the pit, despite this plot being on a $20^{\circ}$ slope. Moisture values in mineral soil layers remained well within the available range throughout the growing season.

Ground water was encountered within the upper $100 \mathrm{~cm}$. of the profile in the Deadwood Creek Plot (P4) only once, in early December, 1951, following the heavy rainfall of November. On this occasion, water flowed into the sampling pit $60 \mathrm{~cm}$. below the soil surface. When the same pit was resampled in early January, about two weeks after snowfall prevented further infiltration, no water was encountered within $100 \mathrm{~cm}$. of the soil surface. The high soil-moisture unit readings of early February, April, and May, 1953, however, indicate that seepage moisture may have been present in the lower profile on these occasions as well. The absence of high values later in the season shows that no further supplements to soil-moisture reserves occurred within the upper $100 \mathrm{~cm}$. Nevertheless, since this stand was adjacent to the Deadwood Creek Thuja-Lysichitum Plot, it is possible that seepage moisture, which came to the surface in the latter stand, also affected deeper layers than those sampled in the PseudotsugaPolystichum Plot. The upper profile, however, appeared little affected by seepage moisture at any time of the year. Aithough moisture values were high in the available range at most samplings, reserves were reduced to quite low levels during the prolonged drought of 1952, and some deficits occurred in the latter part of each season.

During the early months of each growing season, soil-moisture contents were found to be high in the available range in the deep, stone-free profile of the Valley Plot (P3). In dry years, nevertheless, marked deficits were evident later in the season. Values above field capacity were recorded at $125 \mathrm{~cm}$. during October and November, 1953, and each winter, temporary sloughs were present in hollows adjacent to the plot. However, the location of this stand on an old river terrace precluded additions to soil-moisture reserves by ground water during the growing season. This plot was less influenced by secpage moisture than any of the other Pseudotsuga-Polystichum stands sampled and it hat the driest soil-moisture regime. 
Water remained close to the surface of the swampy areas in the ThujaLysichitum plots at all times. This water table was perched above the gley layer beneath the muck. Late in summer water levels dropped 10 or $15 \mathrm{~cm}$., but the muck layer remained close to saturation at all times (Table IX).

Litter layers on the marginal hummocks of the Deadwood Creek and Wolf Mountain Thuja-Lysichitum Plots (Ly2 and Ly3) dried out by the latter part of each summer. Farther west in the Echo Mountain Plot, however, even this layer contained available moisture throughout the growing season. The upper portion of the peaty layers beneath the litter layer showed some moisture deficits during summer months, but values did not drop below wilting percentage. Lower layers remained moist even by late summer and they were saturated during winter months.

\section{DISGUSSION}

\section{Water Relations and the Differentiation of Associations}

The considerable differences in moisture regime between plots representing different associations indicate the importance of water relations in site differentiation. Thus, the small size and low volume per acre of Pseudotsuga menziesii in the Pseudotsuga-Gaultheria-Peltigera plots were correlated with a dearth of available soil moisture during much of the growing season. Moisture deficits were likewise sufficient to account for the low height and density of Gaultheria shallon. The high soil-surface temperatures and rapid evaporation rates which resulted from this openness in tree and shrub canopies doubtless accentuated the droughtiness of superficial soil horizons. Such droughtiness could well be a limiting factor in the survival of ground cover species characteristic of more humid locations. The greater height and volume of Pseudotsuga menziesii and increased density of Gaultheria shallon in the Pseudotsuga-Gaultheria plots were associated with a somewhat less droughty soil-moisture regime. However, this greater density of the tree and shrub layers appeared to limit the abundance of the lichens and mosses characteristic of the ground cover and lower boles of trees in Pseudotsuga-Gaultheria-Peltigera stands. The large volume per acre of Pseudotsuga menziesii in the Pseudotsuga-Polystichum plots was correlated with soils having relatively small moisture deficits during the growing season. On the other hand, infrequency of Pseudotsuga menziesii in the Thuja-Lysichitum plots was coincident with the occurrence of a very high water table at all seasons. Such swampy areas supported an herbaceous flora characteristically restricted to wet locations with a cool, humid atmosphere.

While differences in the floristic composition and productivity of the sites studied were found to be correlated with different soil-moisture regimes, significant differences in soil-nutrient regimes were also evident. The duff-mull character of litter layers, the limited development of $\mathrm{A}_{2}$ horizons, and the moderate acidity of soil $\mathrm{pH}$ values in the Pseudotsuga-Polystichum plots indicate that soilfertility levels were relatively high. On the other hand, the mor-type humus, clear differentiation of $\mathrm{A}_{2}$ horizons, and acid soil $\mathrm{pH}$ values in the PseudotsugaGaultheria plots, for example, suggest that their soils were relatively infertile. The lower site index for Pseudotsuga menziesii of Pseudotsuga-Gaultheria sites seems consequently to result from soil-fertility levels lower than those of Pseudotsuga-Polystichum sites, as well as less favorable soil-moisture regimes. The seepage moisture which augments soil-moisture supplies in PseudotsugaPolystichum sites may also ameliorate the leaching action of the regional climate evident in Pseudotsuga-Gaultheria sites (20). 
Likewise, the composition of subordinate plant layers seemed to be influenced by soil-fertility levels. The absence of Gaultheria shallon, for example, from the mineral soil of the Pseudotsuga-Polystichum plots indicates that other factors than adequate moisture supply limited its distribution. Its presence on decaying wood, but scarcity in stand openings and on clear-cut Pseudotsuga-Polystichum sites suggest that intolerance to the shade conditions prevailing in the dense, twostoried stands characteristic of these sites is not the main obstacle to the spread of this species to Pseudotsuga-Polystichum sites. Evidently such species as Gaultheria shallon, Hylocomium splendens, and Eurhynchium oreganum are sufficiently obligate oxylophytes that their distribution is restricted to less fertile soils than those characteristic of Pseudotsuga-Polystichum sites. Such plants as Polystichum munitum, Achlys triphylla, Tiarella trifoliata, and Mnium insigne, on the other hand, being confined to moist, relatively fertile soils, characterize the Pseudotsuga-Polystichum association. However, their abundance also appears to be somewhat dependent on the humid, shady conditions of the forest floor of Pseudotsuga-Polystichum stands, since most of these species are less numerous in clear-cut areas than they are beneath standing timber (31).

As in the Pseudotsuga-Polystichum plots, the large size of the Pseudotsuga menziesii in the subassociation nudum plots of the Pseudotsuga-Tsuga-Hylocomium association was correlated with relatively favorable soil-moisture conditions. The lack of well-defined $\mathrm{A}_{2}$ horizons and the low frequency of Gaultheria shallon, Hylocomium splendens, and other oxylophytes on the forest floor of these stands suggest that their soils were also quite fertile. However, the paucity of ground-cover plants characteristic of the Pseudotsuga-Polystichum association and the somewhat lower site index for Pseudotsuga menziesii of these plots seem to reflect the occurrence of both slightly larger soil-moisture deficits during the growing season and somewhat less favorable soil-fertility levels than those typical of Pseudotsuga-Polystichum sites.

Site index for Pseudotsuga menziesii in the subassociation typicum plots of the Pseudotusga-Tsuga-Hylocomium association was no higher than it was in the Pseudotsuga-Gaultheria plots. In the easterly typicum plots, this poor growth would appear at least partially attributable to soil drought, since soil-moisture deficits were comparable with those of Pseudotsuga-Gaultheria sites. However, the absence of Gaultheria shallon would imply that other factors were different. Such differences might be related to soil fertility and the fairly dense tree canopy characteristic of Pseudotsuga-Tsuga-Hylocomium stands.

On the other hand, the low site index for Pseudotsuga menziesii in the Fourth Lake Pseudotsuga-Tsuga-Hylocomium Plot (M1) was probably more closely related to poor soil fertility than soil drought, since soil-moisture deficits in this plot were less pronounced than those of the more easterly Deadwood Creek Plot (M4). Despite its relatively droughty soil-moisture regime, the Deadwood Creek Plot had a higher site index for Pseudotsuga menziesii than the Fourth Lake Plot. The deeper $\mathrm{A}_{2}$ horizon and lower $\mathrm{pH}$ values of the westerly plor (M1) indicate that its soils were more strongly leached than those of the easterly plot (M4). Apparently the more favorable soil-moisture regimes of the fourth Lake Plot did not offset a less favorable soil-fertility regime.

The similarity of site index for Pseudotsuga menziesii in Plots M1 and M4 of the Pseudotsuga-Tsuga-Hylocomium association, despite dissimilarities in soil-moisture regime, illustrates the compensatory roles played by soil-nutrient and soil moisture regimes under different climatic conditions. The more favorable soil-moisture regimes of the westerly plots were evidently counterbalanced by more highly leached soils than those of similar vegetation types farther east. 
The improved soil-moisture regimes of the westerly plots probably resulted from both rainfall being higher and the snow pack lasting longer than farther east. Although summer rainfall at lourth Lake was light, it was, nonetheless, nearly double that at Deadwood Creek to the east. Presumably evaporation rates and transpiration usage were also influenced by the greater prevalence of cloudy conditions in the westerly portion of the valley. The long duration of the snow pack on the upper slopes of this mountainous area was probably even more influential than summer rainfall, because melting snow supplied seepage moisture well into the growing season, long after such additions to soil-moisture supply had ceased in the more easterly plots.

Soil-moisture deficits in the Pseudotsuga-Tsuga-Gaultheria Plots at Fourth Lake and on Echo Mountain were somewhat greater than those of the Fourth Lake Pseudotsuga-Tsuga-Hylocomium Plot, and their soils appeared to be even more strongly leached. On Echo Mountain (Plot G2), such conditions resulted in the lowest site index for Pseudotsuga menziesii of any plot. The better growth of Pseudotsuga menziesii in the Fourth Lake Pseudotusga-Tsuga-Gaultheria Plot (G1) may have been the result of subsurface soils in this plot being more influenced by seepage moisture than those of the Echo Mountain Plot (G2). Such seepage presumably brought about the somewhat more favorable soilmoisture regime and it may also have ameliorated to some extent the leaching influence of the wet climate of this portion of the study area. Soil-surface temperatures and evaporation rates within subordinate plant layers show that conditions in the Pseudotsuga-Tsuga-Gaultheria plots were normally cooler and moister than in the more easterly Pseudotsuga-Gaultheria plots. The presence of Chamaecyparis nootkatensis, Gaultheria ovatifolia, Rhytidiopsis robusta, and other species common in relatively high altitude stands, was doubtless related to this difference.

As in the Pseudotsuga-Tsuga-Gaultheria and Pseudotsuga-Tsuga-Hylocomium plots at Fourth Lake, it is likely that the low site index for Pseudotsuga menziesii in Plot L1 of the Pseudotsuga-Gaultheria-Peltigera association resulted as much from the highly leached character of its soils as it did from drought, since moisture deficits were neither so extreme nor prolonged as they were in the easterly plots of this association. However, temperatures and evaporation rates in this opencanopied stand were relatively high, and lichens and drought-tolerant mosses had a frequency of occurrence characteristic of the association. Occasional severe or prolonged droughts might also play a part in reducing the abundance of mesophytes. Most of the shrubby Tsuga heterophylla, which occurred as thickets in this plot, had died by 1959, following the very dry summers of 1952 and 1958 . Greater mortality among Tsuga heterophylla regeneration than in that of Pseudotsuga menziesii has been observed in other stands on similar sites following the very dry summer of 1958 (12). The relatively deep root system of Pseudotsuga menziesii may increase its drought resistance under such conditions.

Some of the Tsuga heterophylla on the Fourth Lake Pseudotsuga-Polystichum Plot were in the same age class as the Pseudotsuga menziesii of the dominant canopy, even though many were younger, since Tsuga heterophylla is quite shade-tolerant. Pseudotsuga menziesii, nevertheless, overtopped by $25 \mathrm{ft}$. or more the $175 \mathrm{ft}$. reached by the tallest Tsuga heterophylla. While the height of all tree species in strongly leached soils was less than in the relatively fertilo soil characteristic of Pseudotsuga-Polystichum sites, the impairment of Tsuga heterophylla in the strongly-leached and periodically moisture-deficient soils of the Echo Mountain Pseudotsuga-Tsuga-Gaultheria Plot seemed comparably less than that of Pseudotsuga menziesii. In this latter plot, Pseudotsuga menziesii averaged only $7 \mathrm{ft}$. taller than the $70 \mathrm{ft}$. averaged by Tsuga heterophylla of the same age class. On the more fertile and moister Pseudotsuga-Polystichum site at 
Fourth Lake the difference was $25 \mathrm{ft}$. in favor of Pseudotsuga menziesii, as previously noted. In more strongly leached soils, such as those of the adjacent Pseudotsuga-Tsuga-Hylocomium Plot (M1), Pseudotsuga menziesii still remained less clearly dominant than it was in more fertile soils, even though soil-moisture deficits were small.

The relative productivity of Pseudotsuga menziesii and Tsuga heterophylla in the easterly plots of the study area was not directly comparable because all the Tsuga heterophylla were in a younger age class than the Pseudotsuga menziesii of the dominant canopy. The ground fires which swept many stands subsequent to their initial establishment might have destroyed any older Tsuga heterophylla formerly present. The abundance of this species might also have been limited by unfavorable conditions for the survival of Tsuga heterophylla seedlings during the initial period of stand establishment (14). Data from stands analyzed by Schmidt (41) in various parts of Vancouver Island, nevertheless, suggest that the volume produced by Pseudotsuga menziesii on high-quality sites, such as those supporting stands of the Pseudotsuga-Polystichum association, is greater than that of Tsuga heterophylla. In the study area, Pseudotsuga menziesii productivity on dry sites seems to have been greater than that of Tsuga heterophylla. However, since the Tsuga heterophylla in the easterly plots were few in number and limited to the secondary canopy, the productivity of this species relative to that of Pseudotsuga menziesii on dry sites can only be surmised. It might be anticipated, nevertheless, that growth rates for Pseudotsuga menziesii would be better than those of Tsuga heterophylla of the same age class because the latter species is shallow-rooted. Presumably shallow-rooted trees growing on dry sites would have access to a smaller volume of available soil moisture than deep-rooted species.

Growth rates for Thuja plicata, another shallow-rooted species, likewise seemed impaired by a droughty soil-moisture regime. This species, moreover, seems to be more adversely affected by highly-leached soils than Tsuga heterophylla. The greatest productivity of Thuja plicata in the Douglas-fir region is reported to be in the quite fertile and occasionally flooded soils of PseudotsugaThuja-Adiantum sites (20). The continuously high water table of Thuja-Lysichitum sites appears to be somewhat less than optimum even for Thuja plicata.

\section{Water Relations and Forest Distribution}

Since water relations evidently exert a considerable influence in the differentiation of the associations studied, factors which regulate water relations would seem of importance in forest distribution. In a region where the climate is characterized by potential moisture deficiencies during the growing season, topography and the depth and texture of the soil profile seem to be the most influential regulators of soil-moisture regimes. The catenary distribution of forest associations occurring in the Douglas-fir region on Vancouver Island is evidently a consequence of these factors. Thus, ridges support stands of the Pseudotsuga-Gaultheria-Peltigera association partly because ground water does not supplement soil-moisture reserves in such topographic positions and partly because moisture reserves are commonly limited by shallow soil depth and coarse texture. Where greater soil depth results in a slightly larger soil-moisture storage capacity, Pseudotsuga-Gaultheria sites occur, even though soil texture may be equally coarse. Furthermore, on slopes, seepage-moisture flow, even if of short duration, may contribute to the improvement in water relations which allows the development of Pseudotsuga-Gaultheria stands. Lower slopes evidently support Pseudotsuga-Polystichum stands because seepage moisture from upper slopes, supplementing moisture reserves, results in favorable soil-moisture 
regimes. Such supplements are especially effective when relatively fine soil texture promotes the influence of seepage moisture even in the superficial layers of soil profiles. On the other hand, it seems likely that Pseudotsuga-TsugaHylocomium sites occur at mid-slope because seepage moisture there is insufficiently sustained to maintain such favorable soil-moisture or fertility levels. In locations where seepage water is so abundant that it comes to the surface, swampy Thuja-Lysichitum sites are formed, as long as this water remains moving and does not become stagnant. Such sites are essentially independent of rainfall for the recharge of their moisture reserves.

The influence of soil texture on stand distribution is well illustrated by the occurrence of either typicum or nudum stands of the Pseudotsuga-Tsuga-Hylocomium association at mid-slope according to soil-parent material. Where soils are derived from coarse-textured materials, seepage has relatively little influence on the upper soil profile and typicum sites occur. Such sites have quite strongly leached and droughty upper-soil horizons, despite the occurrence of seepage moisture in the lower profile. On the other hand, finer-textured soils normally support nudum stands, apparently because moisture reserves are then greater and seepage has more influence, both through supplementing soil-moisture supplies and ameliorating the leaching influence of the climate.

Even greater site differences can occur when the secondary relief of hillsides diverts the flow of seepage moisture. The Echo Mountain Pseudotsuga-TsugaHylocomium Plot (M2), for example, was located on a concave portion of the main slope, which was influenced by seepage moisture at least into early summer. On the other hand, the coarse-textured soil of the adjacent Pseudotsuga-TsugaGaultheria Plot (G2), which was on a convex shoulder of the same slope less than one-half mile away and only $100 \mathrm{ft}$. higher in elevation, was much less influenced by seepage. The soils of this latter plot consequently were drier in summer and more strongly leached than those of Plot M2. The difference of $110 \mathrm{ft}$. in site index for Pseudotsuga menziesii would seem largely a consequence of this diversion of seepage moisture.

Minor variations in relief and differences in soil-parent material and soil depth may also modify moisture regime and stand distribution on the relatively level terrain of valley floors. The Pseudotsuga-Polystichum Plot (P4) on the valley floor of Deadwood Creek, for example, was situated on fairly deep outwash material which remained moist during much of the growing season. On the other hand, the coarse-textured, glacial-till soil of a nearby moraine was droughty because it was uninfluenced by ground water during the growing season and moisture reserves were small. This slight ridge supported a stand of the Pseudotsuga-Gaultheria association (Plot G6). An adjacent Pseudotsuga-Gaultheria-Peltigera site (Plot L3) was still more droughty because there the ortstein layer was even closer to the surface. Conversely, even though Plot P3 of the Pseudotsuga-Polystichum association, which was situated on an old river terrace in the main valley of the Nanaimo River, was likewise uninfluenced by ground water during the growing season, its relatively deep, fine-textured profile had enough moisture-storage capacity to provide available moisture for a considerable proportion of the growing season. The recent alluvium which formed the parent material of this plot seemed to be fertile as well. However, lack of seepage moisture resulted in this plot having a drier soil-moisture regime than the other Pseudotsuga-Polystichum sites analyzed and this difference presumably accounts for the relatively high frequency of Achyls triphylla. As on the valley floor of Deadwood Creek, nearby glacial-till soils supported stands of the PseudotsugaGaultheria or Pseudotsuga-Gaultheria-Peltigera associations.

While the ortstein layer present in many profiles merely restricts the depth of the root zone and limits soil-moisture storage capacity, such impervious 
layers also play an important role in seepage-water movement. Without impervious bedrock, ortstein, clay, or gleyed layers, gravitational water could penetrate to considerable depths, as occurs, for example, in deep gravel soils or soils derived from shales having their cleavage planes normal to the soil surface; much drier sites than those characteristic of lower slopes in the study area might then occur. The gross distribution of associations is accordingly a catenary sequence of stands largely because seepage water remains within the root zone. There, such water can effectively influence water relations through its influence on soil-moisture regimes, a manifestly important factor in forest distribution because the climate of the Douglas-fir region is potentially moisture deficient during the growing season. Nevertheless, it is evident that secondary topography, soil-parent material, and soil depth can modify this catenary sequence because these factors also play an important role in the determination of soilmoisture and nutrient regimes.

\section{SUMMARY}

The climate, vegetation, microclimate, soil profiles, and soil-moisture regimes of 24 plots were analyzed to evaluate the influence of water relations on forest distribution in the Douglas-fir region on Vancouver Island, British Columbia. Plots were located in mature stands representative of six forest associations in the Nanaimo River Valley.

Species representation and the abundance and vigor of plants were found to be correlated with differences in soil-moisture regime and atmospheric humidity. The low site index for Pseudotsuga menziesii and stunted growth of Gaultheria shallon in Pseudotsuga-Gaultheria-Peltigera plots were associated with a reduction of soil-moisture content to the wilting range for extended periods during the growing season and with relatively arid atmospheric conditions. The somewhat higher site index for Pseudotsuga menziesii and taller Gaultheria shallon of Pseudotsuga-Gaultheria plots were correlated with less extreme soil-moisture deficiencies. In Pseudotsuga-Polystichum plots, soil-moisture contents remained high in the available range during much of the growing season. Such moist and relatively fertile sites had a high site index for Pseudotsuga menziesii and characteristic mesophytes were frequent in subordinate plant layers. Thuja plicata, rather than Pseudotsuga menziesii, was the most abundant tree in ThujaLysichitum plots and other characteristic species were typical of the non-stagnant, swampy parts of these stands. A consistently high water table and humid atmosphere vere characteristics of such sites.

The low site index for Pseudotsuga menziesii in Pseudotsuga-Tsuga-Gaultheria plots seemed to result as much from the highly leached character of their soils as it did from lack of available water, since the soils of these plots were moister during much of the growing season than those of Pseudotsuga-Gaultheria sites. Similarly, the relatively moist, but quite highly leached, soil of a subassociation typicum plot of the Pseudotsuga-Tsuga-Hylocomium association had a lower site index for Pseudotusga menziesii than plots with droughtier but less highly leached soils.

Since rainfall supplies little moisture during the growing season in this rainshadow region, two influential factors regulating soil-moisture regimes are the moisture-storage capacity of soil profiles and the extent of influence of seepage. The catenary distribution of stands typical of hillsides seems to reflect the sequence of soil-moisture regimes controlled by these factors. It was concluded that ridges are droughty partly because their topographic position precludes ground water from supplementing moisture stored in their profiles and partly because such reserves are usually limited by shallow soil depth and 
coarse texture. Farther downslope, both seepage from upper slopes and deeper soils normally result in moister soils and a higher site index for Pseudotsuga menziesii. Tariation in the extent of coverage by the different stands in the typical sequence of associations occurs, nevertheless, since differences in local topography and soil characteristics influence soil-moisture regimes.

\section{ACKNOWLEDGMENTS}

The athor wishes to acknowledge the assistance given him by the many individuals and organizations who helped in the completion of this study. Thanks are especially due to Dr. Y. J. Krajina, Professor of Forest Ecology, for his advice and encouragement, to Dr. T. M. C. Taylor, Head of the Department of Biology and Botany, Dr. A. H. Hutchinson, formerly Head of the Department of Biology and Botany, Dr. G. S. Allen, Dean of the Faculty of Forestry, and Dr. C. A. Rowles, Head of the Soils Department, all of the University of British Columbia. He is also grateful to Dr. R. Daubenmire of the Botany Department, Washington State University, for his helpful criticism of the thesis manuscript upon which this paper is based.

The support of the Comox Logging Company Ltd. and the late Mr. F. D. Mulholland, formerly their Chief Forester; the National Research Council, Ottawa, Ontario; the Department of Veterans Affairs, Ottawa, Ontario; the Alaska Pine Company, Ltd., Vancouver, B.C.; the British Columbia Electric Company, Ltd., Vancouver, B.C.; and the British Columbia Sugar Refining Company, Ltd., Vancouver, B.C. is gratefully acknowledged.

Assistance and data were also given by Dr. R. E. Foster, Head of Forest Pathology Investigations, and other members of the staff of the Forest Biology Laboratory, Victoria, B.C.; Mr. W. H. Mackie, Meteorologist-in-Charge, Gonzales Observatory, Victoria, B.C.; Mr. W. Panczyszyn, British Columbia Forest Products Company, Ltd., Youbou, B.C.; the Empress Manufacturing Company, Ltd., Vancouver, B.C.; Miss M. A. Allen; Mr. W. Arlidge; Dr. T. C. Brayshaw; Mr. R. B. Dickens; Mr. L. Farstad; Mr. T. Lord; Mrs. G. M. McMinn; Mrs. R. G. McMinn; Dr. D. Mueller-Dombois; Mr. F. Rayer; Mr. R. L. Schmidt; Mr. R. H. Spilsbury; Dr. A. F. Szczawinski; Mr. G. Tilser; and Dr. W. G. Wellington. 


\section{REFERENCES}

1. American Society for Testing Materials. 1944. Procedures for testing soil; standard method of mechanical analysis for soils. Amer. Soc. Test. Materials, Philadelphia, Pa.

2. Becking, R. W. 1956. Die natürlichen Douglasien-Waldgesellschaften Washingtons und Oregons. Allgemeine Forst und Jadgzeitung, $1956(2 / 3): 42-56$.

3. Briggs, L. J. and Shantz, H. L. 1917. Comparison of the hourly evaporation rate of atmometers and free water surfaces with the transpiration rate of Medicago sativa. J. Agr. Research, 9: 277-292.

4. Braun-Blanquet, J. 1951. Pflanzensoziologie. 2nd ed. Springer-Verlag, Wien.

5. CANFIELD, R. H. 1941. Application of line interception method in sampling range vegetation. J. Forestry, 39: 388-394.

6. Chapman, J. D. 1952. The climate of British Columbia. In Trans. 5th B.C. Nat. Res. Conf., Victoria.

7. Colman, E. A. and Hendrix, T. M. 1949. The fiberglas electrical soil-moisture instrument. Soil Sci. $67: 425-438$.

8. Conard, H. S. 1944. How to know the mosses. Wm. C. Brown Co., Dubuque, Iowa.

9. Daubenmire, R. 1947. Plants and environment. John Wiley and Sons, Inc., New York, N.Y.

10. DaY, J. H., FARstad, L., and Laird, D. 1959. Soil survey of southeast Vancouver Island and Gulf Islands, British Columbia. Canada Dept. Agric., (co-op. Univ. B.C. and B.C. Dept. Agric.) B.C. Soil Survey, Report No. 6.

11. Fink, B. 1935. Lichen flora of the United States. Univ. Press, Univ. Michigan, Ann Arbor, Mich.

12. Foster, R. E. Unpublished.

13. Forest Surveys Division. 1955. Interim standard cubic-foot volume tables for the commercial tree species of British Columbia. B.C. Forest Service. Multigraphed.

14. Garman, E. H. 1955. Regeneration problems and their silvicultural significance. B.C. Forest Service, Tech. Publ. T. 41.

15. Geiger, R. 1950. The climate near the ground. Harvard Univ. Press, Cambridge, Mass.

16. Hendrix, T. M. and Colman, E. A. 1951. Calibration of fiberglas soil-moisture units. Soil Sci. 71: 419-427.

17. Hoover, M. D. and Lunt, H. A. 1952. A key for the classification of forest humus types. Proc. Soil Sci. Soc. Amer. 16: 368-370.

18. Howard, Grace, E. 1950. Lichens of the State of Washington. Univ. Wash. Press, Seattle, Wash.

19. Krajina, V. J. 1933. Die Pflanzengesellschaften des Mlynica-Tales in den Vysoke Tatry (Hohe Tatra). Beihefte zum Botanischen Centralblatt., Bd. L, Abtlg. II, 774-957 (I. Teil); Bd. LI, Abtlg. II, 1-224 (II. Teil).

20. Krajina, V. J. The ecological classification of the forests of the eastern part of Vancouver Island. Unpublished.

21. Krajina, V. J. 1959. Bioclimatic zones in British Columbia. Univ. British Columbia, Botanical Series No. 1, Vancouver.

22. Krajina, V. J. and Spilsbury, R. H. Unpublished.

23. Kramer, P. J. 1949. Plant and soil water relationships. McGraw-Hill Book Co., Inc., New York, N.Y.

24. Levr, E. B. and Madpen, E. A. 1933. The point method of pasture analysis. New Zealand J. Agr. 46: 267-279.

25. Littue, E. L. 1953. Check list of native and naturalized trees of the United States (including Alaska). U.S. Dept. Agr., Agr. Handbook No. 41.

26. Livingston, B. E. 1935. Atmometers of porous porcelain and paper; their use in physiological ecology. Ecology, 16: 438-472.

27. Lugo-Lopez, M. A. 1952. Comparative value of various methods of approximating the permanent wilting percentage. J. Agr., Univ. Puerto Rico, 36(2): 122-133.

28. McArdee, R. E., Meyer, W. H., and Bruce, D. 1949. The yield of Douglas fir in the Preific Northwest. U.S. Dept. Agr., Tech. Bull. 201. 
29. MCMINx, R. G. 1952. The role of soil drought in the distribution of vegetation in the northern Rocky Mountains. Ecology, 3:3: 1-1j.

30. Mindleton, IV. W. D. 19) Meteorological instruments. Univ. Toronto Press, Toronto, Ont.

31. Muedefi-1)ombois, 1). 1959. Douglas-fir forest associations on Vancouver Island in their initial stages of secondary succession. Univ. British Columbia, Ph. D. Thesis.

32. PEARS N, G. A. 1931. Forest types in the southwest as determined by climate and soil. U.S. Dept. Agr. Tech. Bull. 247.

33. PECk, M. E. 1941. A manual of the higher plants of Oregon. Binsford and Mort, Portland, Oregon.

34. Richards, I. A., ed. 1954. Diagnosis and improvement of saline and alkali soils. U.S. Dept. Agl., Agr. Handbook 60 .

35. Riciarus, L. A. and Wadeigh, C. H. 1952. Soil water and plant growth. In Soil physical conditions and plant growth. ed. B. T. Shaw. Academic Press Inc., New York, N.Y.

36. Richards, L. A. and Weaver, L. R. 1943. Fifteen-atmosphere percentage as related to permanent wilting percentage. Soil Sci. 56: 331-339.

37. Richards, L. A. and Weaver, L. R. 1944. Moisture retention by some irrigated soils as related to soil-moisture tension. J. Agr. Research, 69: 215-235.

38. Riesbol, H. S. 1938. Results from experimental rain-gages at Coshocton, Ohio. Trans. Amer. Geophys. Union, 1938: 542-550.

39. Rowe, J. S. 1959. Forest regions of Canada. Canada Dept. Northern Affairs and Nat. Res. Forestry Branch, Bull. 123.

40. Schmid, R. L. 1957. The silvics and plant geography of the genus Abies in the coastal forests of British Columbia. B.C. Forest Service, Tech. Publ. T. 46.

41. Sснмidt, R. L. Unpublished.

42. Simti, W. H. and Moodie, C. D. 1947. Collection and preservation of soil profiles. Soil Sci. 64: 61-69.

43. Thornthwaite, C. W. and Mather, J. R. 1955. The water balance. Drexel Inst. Technology, Lab. Climatology, Publ. Climatology 8(1).

44. United States Department of Agriculture. 1951. Soil survey manual. U.S. Dept. Agr., Agr. Handbook 18.

45. Wrlde, S. A. 1954. Forest humus; its genetic classification. Trans. Wisconsin Acad. Sci., Arts and Lett. 43: 137-163.

46. Wilde, S. A. 1958. Forest soils. The Ronald Press Co., New York, N.Y. 
APPENDIX I

1951-56 MEANS COMPARED WITH LONG-TERM MEANS FOR PRECIPITATION AND TEMPERATURE AT VARIOUS WEATHER STATIONS ON VANCOUVER ISLAND

\begin{tabular}{|c|c|c|c|c|c|c|c|c|}
\hline & \multicolumn{2}{|c|}{ Pachena Point } & \multicolumn{2}{|c|}{ Nitinat Camp } & \multicolumn{2}{|c|}{ CoWICHAN LAKE } & \multicolumn{2}{|c|}{ DUNCAN } \\
\hline & $\begin{array}{c}31 \text { year } \\
\text { mean }\end{array}$ & $\begin{array}{l}1951-56 \\
\text { mean }\end{array}$ & $\begin{array}{l}9 \text { year } \\
\text { mean }\end{array}$ & $\begin{array}{c}1951-56 \\
\text { mean }\end{array}$ & $\begin{array}{l}40 \text { year } \\
\text { mean }\end{array}$ & $\begin{array}{l}1951-56 \\
\text { mean }\end{array}$ & $\begin{array}{l}30 \text { year } \\
\text { mean }\end{array}$ & $\begin{array}{l}1951-56 \\
\text { mean }\end{array}$ \\
\hline Precipitation (inches) & & & & & & & & \\
\hline $\begin{array}{l}\text { Annual mean } \ldots \ldots \ldots \ldots \ldots \ldots \ldots \ldots \\
\text { Summer }{ }^{1} \text { mean } \ldots \ldots \ldots \ldots \ldots \ldots \ldots\end{array}$ & $\begin{array}{r}111 \\
9.5\end{array}$ & $\begin{array}{r}125 \\
10.4\end{array}$ & $\begin{array}{r}113 \\
5.8\end{array}$ & $\begin{array}{r}121 \\
6.0\end{array}$ & $\begin{array}{r}72 \\
3.8\end{array}$ & $\stackrel{88}{5.2}$ & $\begin{array}{l}39 \\
3.2\end{array}$ & $\begin{array}{l}43 \\
3.2\end{array}$ \\
\hline Temperature $\left({ }^{\circ} \mathrm{F} .\right)^{2}$ & & & & & & & & \\
\hline $\begin{array}{l}\text { Summer mean monthly maxima... } \\
\text { Summer mean monthly ranges.... } \\
\text { Winter }{ }^{4} \text { mean monthly minima... } \\
\text { Winter mean monthly ranges..... }\end{array}$ & $\begin{array}{l}72 \\
30 \\
25 \\
27\end{array}$ & $\begin{array}{l}70 \\
28 \\
24 \\
26\end{array}$ & $\overline{-}$ & $\overline{-}$ & $\begin{array}{l}87^{3} \\
47 \\
17 \\
31\end{array}$ & $\begin{array}{l}85 \\
44 \\
18 \\
30\end{array}$ & $\begin{array}{l}91 \\
49 \\
17 \\
37\end{array}$ & $\begin{array}{l}93 \\
44 \\
20 \\
33\end{array}$ \\
\hline
\end{tabular}

1Summer: June, July, and August.

${ }^{2}$ Mean temperatures for the years 1942-55 only.

${ }^{3}$ Mean temperatures for the years 1950-56 only.

${ }^{4}$ Winter: December, January, and February. 


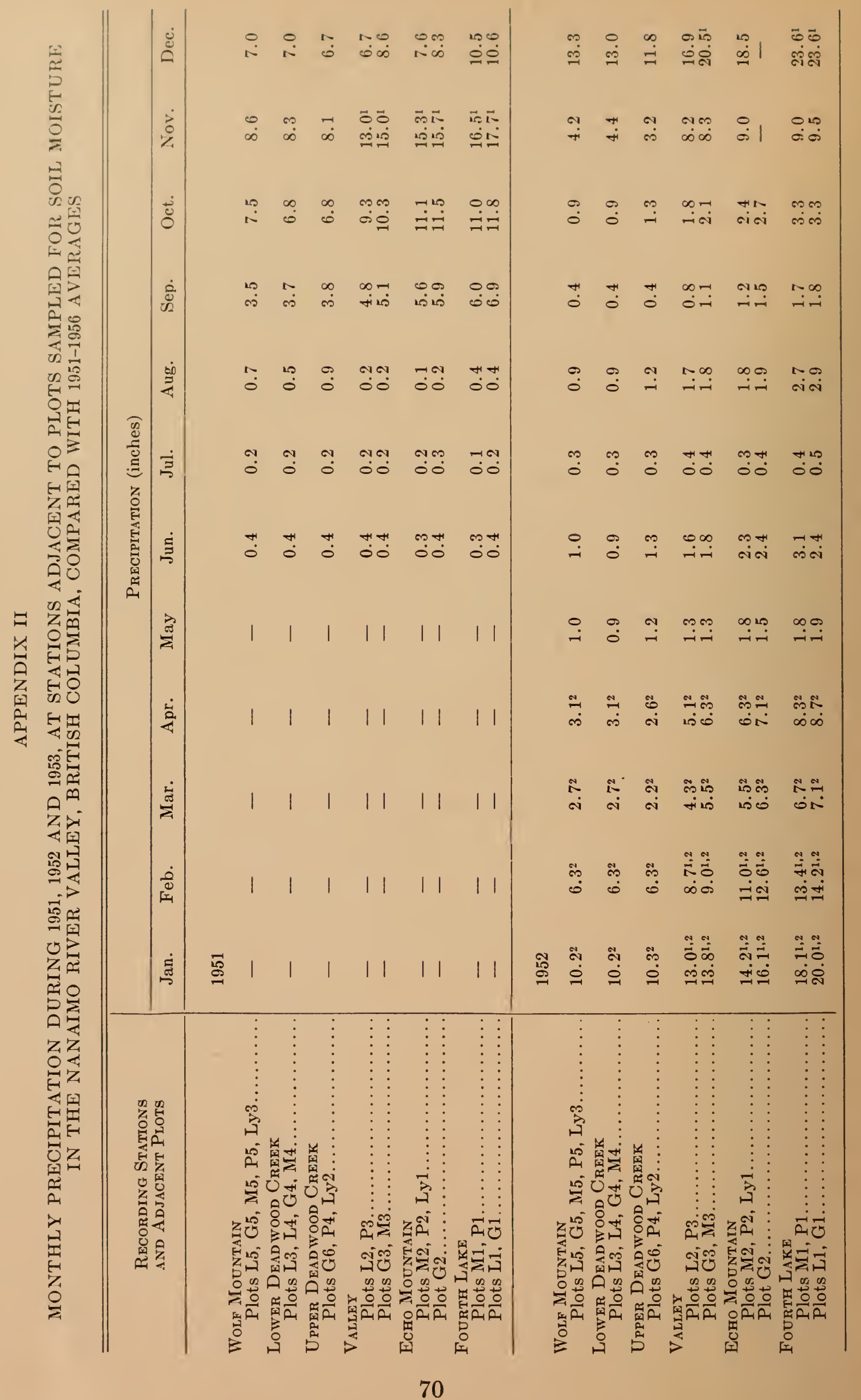




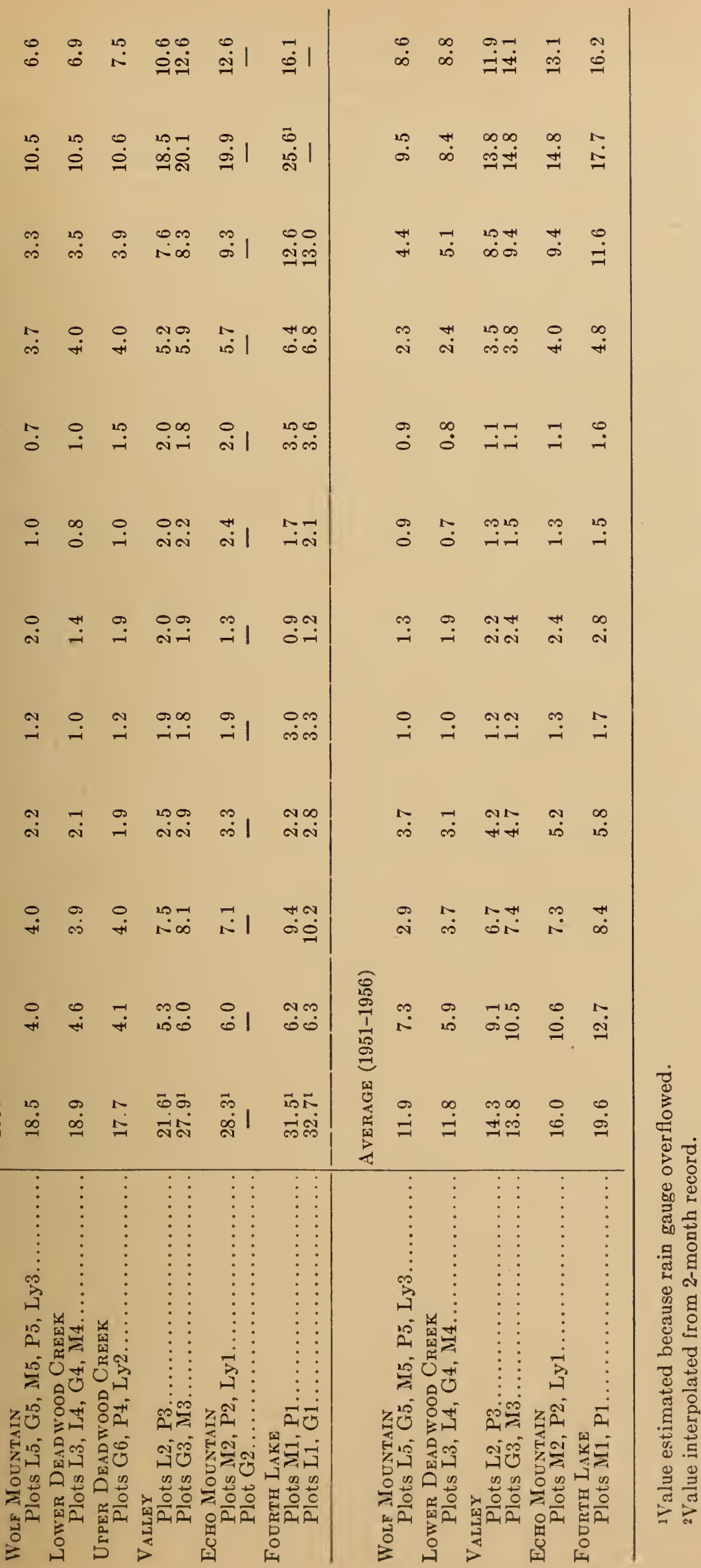




